UNIVERSIDADE DE SÃo PAULO

Instituto de Física

\title{
Núcleos Transicionais na Região de $\mathrm{A} \approx 130$
}

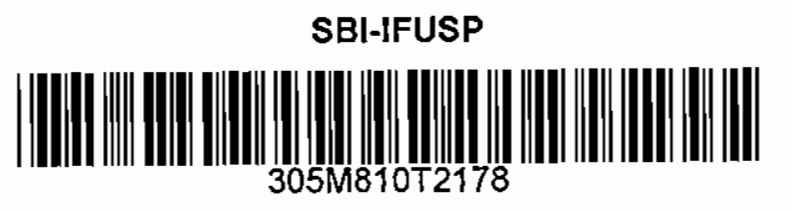

Márcia de Almeida Rizzutto

Tese apresentada ao Instituto de Física da Universidade de São Paulo para obtenção do título de Doutor em Ciências.

Comissão Examinadora:

Profa. Dra. Ewa Wanda Cybulska (IFUSP)

Prof. Dr. Celso Luiz lima (IFUSP)

Prof. Dr. Otaviano Helene (IFUSP)

Prof. Dr. Paulo Roberto S. Gomes (UFF)

Prof. Dr. Luiz Felipe Canto (UFRJ)

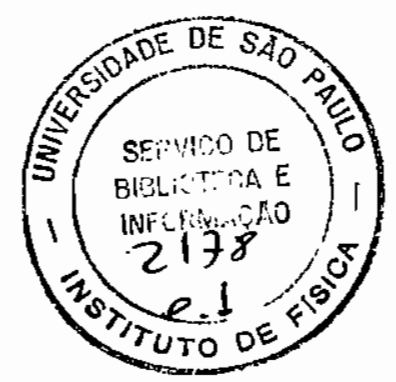

Orientadora

Profa. Dra. Ewa Wanda Cybulska
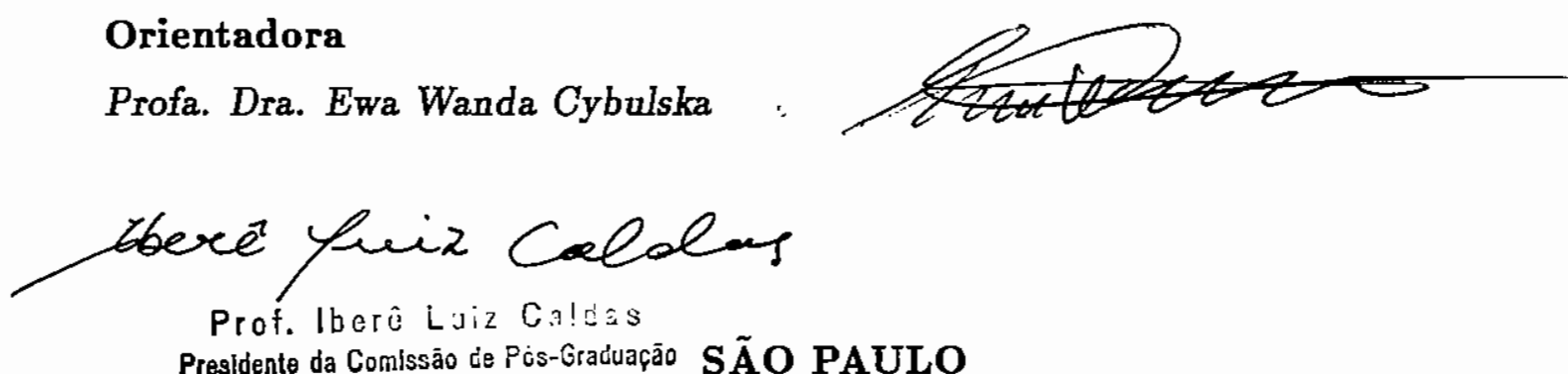

Prof. Iberónto da Comissão de Pós-Graduação SÃO PAULO 


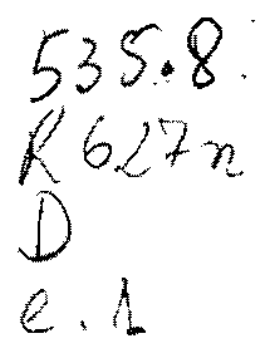

FICHA CATALOGRÁFICA

Preparada pelo Serviço de Biblioteca e Informaçāo do Instituto de Física da Universidade de São Paulo

Rizzutto, Márcia de Almeida

Núcleos transicionais na região de $A \cong 130$. São Paulo, 1993.

Tesé (Doutorado) - Universidade de São Paulo. Instituto de Física. Departamento de Física Nuclear.

Área de Concentração: Física Nuclear

Orientador: Profa. Dra. Ewa Wanda Cybulska

Unitermos: 1. Espectroscopia $\gamma$; 2. Reações de fusão evaporação; 3. ${ }^{138}$ Pr; 4. Coincidência $\gamma-\gamma-t ; 5$. Função de excitação; 6. Distribuição angular; 7. Modelo de "Cranking"; 8. "Projected Shell Model"; 9. Alto spin. 
Aos meus pais

ao Reinaldo

e ao Felipe 


\section{Abstract}

With the view of extending the systematics of the odd-odd, $A=130-140$ mass region nuclei towards the $\mathrm{N}=82$ closed shell, high spin states in ${ }^{138} \mathrm{Pr}$ nucleus have been investigated with the ${ }^{128} \mathrm{Te}\left({ }^{14} \mathrm{~N}, 4 \mathrm{n} \gamma\right)$ reaction, using on-line $\gamma$-ray spectroscopy techniques. For the first time three rotational bands were observed in this nucleus. A theoretical analysis of the mass region $A=130$ - 140 was carried out with the Cranking Shell Model and the Projected Shell Model. Generally speaking, a good agreement was obtained between the experimental and the theoretical results. The first model indicates a shape coexistence in the ${ }^{138} \operatorname{Pr}(\mathrm{N}=79)$ nucleus, while the second one shows an oblate shape. On the other hand, both models suggest a shape transition from prolate $(N=73)$ to oblate $(\mathrm{N}=79$ ) passing through a triaxial region around $\mathrm{N}=77$. 


\section{Resumo}

Com o objetivo de ampliar o conhecimento da estrutura dos núcleos duplamente ímpares da regiāo de massa $A=130$ - 140, foram medidos os estados de spin elevado do ${ }^{138} \mathrm{Pr}$, que se aproxima da camada fechada $\mathrm{N}=82$, através de tećnicas de espectroscopia $\gamma$ em linha utilizando a reação ${ }^{128} \mathrm{Te}\left({ }^{14} \mathrm{~N}, 4 \mathrm{n} \gamma\right)$. Pela primeira vez, três bandas rotacionais foram observadas neste núcleo. Uma análise teórica da sistemática desta regiāo foi feita com base nos modelos de "Cranking Shell Model" e "Projected Shell Model". De um modo geral, uma boa concordância foi obtida entre os resultados experimentais e teóricos. $\mathrm{O}$ primeiro modelo indica a coexistência de formas no ${ }^{138} \operatorname{Pr}(\mathrm{N}=79)$, enquanto o segundo sugere forma unicamente oblata. Por outro lado, os dois modelos prevêm uma transição de forma de prolato $(\mathrm{N}=73)$ para oblato $(\mathrm{N}=79)$ passando por uma região de triaxialidade em torno de $\mathrm{N}=77$. 


\section{Agradecimentos}

Gostaria de expressar os meus sinceros agradecimentos a todas as pessoas que contribuíram na realizaçāo deste trabalho, em particular,

à Profa. Ewa pelo apoio constante durante todas as etapas deste trabalho.

aos colegas do grupo $\gamma$ : José Roberto (Zero), Luiz Guilherme, Medina, Suzana

e Prof. ${ }^{e s}$ Ribas, Juan Carlos, Rao, Wayne e Vito pelo incentivo e ajuda na tomada de dados.

ao Luíz Marcos pela leitura cuidadosa da tese.

ao Fernando pela ajuda na edição da tese.

aos Prof. ${ }^{e s}$ K. Hara e C. Lima pelas valiosas discussões.

ao Prof. O. Sala, por ter colocado à nossa disposição o acelerador Pelletron.

a todos os funcionários do laboratório Pelletron pelo suporte indispensável para a realização deste trabalho.

à FAPESP pelo apoio financeiro. 


\section{Índice}

1 Introdução 1

2 Modelos Teóricos 5

2.1 "Cranking Shell Model" . . . . . . . . . . . . . . . 5 5

2.1.1 Transformaçāo dos Dados Experimentais para o Sistema Intrínseco . 8

2.2 Modelo de Camadas com Projeção de Momento Angular . . . . . . . . . . . 12

2.2 .1 Revisão da Teoria . . . . . . . . . . . . . . 13

3 Parte Experimental $\quad 19$

3.1 Introdução. . . . . . . . . . . . . . . . . . . 19

3.2 Procedimento Experimental e Equipamentos . . . . . . . . . . . . . . 21

3.2 .1 Arranjo Experimental . . . . . . . . . . . . . . . 21

3.2 .2 Eletrônica e Aquisição de Dados . . . . . . . . . . . 25

3.3 Redução dos Dados Experimentais . . . . . . . . . . . . 30

3.3.1 Função de Excitação e Distribuição Angular . . . . . . . . . . . . . 30

3.3.2 Coincidência $\gamma-\gamma-t \ldots \ldots \ldots \ldots \ldots \ldots$

3.3.3 Código Panoramix . . . . . . . . . . . . . . 39

4 Resultados Experimentais e Teóricos $\quad 47$

4.1 Esquema de Níveis do ${ }^{138} \operatorname{Pr} \ldots \ldots \ldots \ldots \ldots \ldots \ldots$

4.1.1 Configuraçōes e Spins das Bandas . . . . . . . . . . 50

4.2 Cálculos de CSM . . . . . . . . . . . . . . . . 57 
4.3 Cálculos com PSM $\ldots \ldots \ldots \ldots \ldots \ldots$

5 Sistemática da Região de Massa $A=130 \quad 75$

5.1 Sistemática com CSM . . . . . . . . . . . . . . . 77

5.2 Sistemática com PSM . . . . . . . . . . . . . . 84

$\begin{array}{lll}6 & \text { Discussão e Conclusão } & 101\end{array}$

$\begin{array}{lll}7 & \text { Referências } & \mathbf{1 0 5}\end{array}$ 


\section{Capítulo 1}

\section{Introdução}

Atualmente, já é bem conhecido, a partir da teoria [1, 2] e de dados experimentais [3, 4], que os núcleos de transição na região de massa $A=130$ - 140 se apresentam como rotores de pouca rigidez quanto à mudança de deformação, isto é, eles são facilmente deformáveis em relação ao ângulo que mede a triaxialidade na representação polar das deformaçōes quadrupolares (núcleos preditos como " $\gamma$-soft"). É também sabido que suas formas são fortemente influenciadas por quasi-partículas em orbitais de alto $\mathrm{j}$ [5], tais como $h_{11 / 2}$, e que estas quasi-partículas de valência dirigem a forma nuclear para valores específicos de gama que dependem da posição do nível de Fermi.

A regiăo de massa $A=130$ - 140 é de grande interesse, principalmente devido às características especiais de seus núcleos, isto é, o nível de Fermi do próton de valência está localizado no início da camada $h_{11 / 2}$ com projecão de momento angular pequeno ( $\Omega$ baixo) favorecendo formas polares axialmente simétricas, $\gamma \approx 0^{\circ}$. Por outro lado, o nível de Fermi do nêutron que está na parte superior da mesma camada ( $\Omega$ alto), tende a produzir deformaçāo $\gamma \approx-60^{\circ}$, a qual representa formas nucleares coletivamente oblatas, na convenção de Lund [6]. Esta competição entre as forças de polarização das quasipartículas ímpares é que pode levar os núcleos a terem formas triaxiais. Devido a estas características houve recentemente uma avalanche de publicaçōes sobre estados de alto spin em núcleos ímpares e ímpar-ímpar nesta região. 
Estudos espectroscópicos de altos spins mostram que os núcleos de $\mathrm{Z}$ ímpar, possuem bandas desacopladas com configuração $h_{11 / 2}$ e simetria axial $\left(\gamma \approx 0^{\circ}\right)$, como por exemplo ${ }^{135,137} \operatorname{Pr}[7,8]$ e ${ }^{133} \mathrm{La}[9]$. Nos núcleos ímpares com $\mathrm{N} \leq 77, \mathrm{Ce}$, Nd e $\mathrm{Sm}$ observam-se bandas com características fortemente perturbadas devido ao nêutron de valência no orbital $h_{11 / 2}(\Omega=9 / 2$ ou 11/2). Já para $N=79$ estas bandas se tornam completamente desacopladas com seqüência $\Delta I=2[10,11,12,13]$. Estas características foram interpretadas como sendo uma transição de forma de prolata para oblata em torno de $\mathrm{N}=78$.

Neste trabalho, foram feitas medidas experimentais de alto spin do núcleo de ${ }^{138} \mathrm{Pr}$ $(N=79)$. Dentro do contexto de um estudo sistemático de núcleos ímpar-ímpar realizado pelo grupo Gama na região de $\mathrm{A}=130-140[3,4,14]$, o presente trabalho tem como principal objetivo o estudo da sistemática de mudança de forma nuclear nesta regiāo. 0 estudo experimental da estrutura nuclear de ${ }^{138} \mathrm{Pr}_{\mathrm{r}}$ visa complementar as informaçōes já existentes, por se tratar de um núcleo que se aproxima da camada fechada de nêutrons $(\mathrm{N}=82)$.

A maior parte dos dados experimentais desta região de massa foram interpretados segundo o modelo de "Cranking Shell Model" (CSM). A aproximação de "cranking" é, em princípio, uma aproximação clássica e fenomenológica e sua vantagem está na sua simplicidade. A idéia básica deste modelo está no fato de que o movimento rotacional coletivo dos núcleons pode ser descrito através da transformação para o sistema de coordenadas que gira com velocidade $\omega$ em torno do eixo de rotação. Os resultados dos cálculos de routhianos totais (energia de excitação no sistema intríseco) mostram uma tendência para a forma prolata com triaxialidade pequena para os núcleos ímpar-ímpar desta regiāo.

Além deste modelo fenomenológico, foi utilizado também o modelo de camadas com projeção de momento angular denominado "Projected Shell Model" (PSM) [15] para a interpretação dos dados experimentais do ${ }^{138} \mathrm{Pr}$. Este modelo, desenvolvido recentemente, é puramente quântico e é baseado em uma hamiltoniana microscópica, interações esquemáticas de dois corpos e projeção em bom momento angular. Os cálculos realizados com PSM para o núcleo de ${ }^{138} \mathrm{Pr}$ e para vizinhos ímpares e ímpar-ímpar desta região mostraram definitivamente uma mudança de forma, de prolata para oblata, em função do 
acréscimo do número de nêutrons.

Uma descrição destes modelos é apresentada no capítulo 2, onde é dada uma ênfase maior ao novo modelo de camadas com projeçāo de momento angular ("Projected Shell Model"). Os detalhes e os procedimentos experimentais de cada medida realizada são apresentados no capítulo 3, enquanto que os resultados obtidos e a comparação com os cálculos teóricos são mostrados no capítulo 4. No capítulo 5 é feito um estudo sistemático de toda esta regiāo de massa. Finalmente, no capítulo 6 é feita uma discussão crítica dos modelos utilizados nesta região e são apresentadas as conclusões deste trabalho.

Em dezembro de 1992 os resultados experimentais do núcleo de ${ }^{138} \operatorname{Pr}$ foram publicados na revista alemã Z. Physik A [16] e a análise teórica deste núcleo, juntamente com a sistemática da região feita através do modelo de "Projected Shell Model" foi submetida, em novembro de 1992, à revista Nuclear Physics A, e aceita para publicação [17]. 


\section{Capítulo 2}

\section{Modelos Teóricos}

\section{1 "Cranking Shell Model"}

O modelo "Cranking Shell Model" tem sido freqüentemente utilizado na interpretação de dados experimentais de estados nucleares de alto spin na região de massa $A=130$ - 140 . Este modelo descreve o movimento da partícula de valência em um núcleo em rotação, com freqüência angular $\omega$, ou seja, escolhe-se um sistema de referência que roda no espaço com velocidade angular $\omega$ em torno de um eixo principal do elipsóide (sistema intrínseco). Conseqũentemente, fica eliminada a dependência temporal do potencial médio [18]. Neste sistema intrínseco, $I_{x}$ é a projeção do momento angular total sobre o eixo de rotação (x). A teoria de CSM considera a freqüência angular como uma quantidade física proporcional a $\frac{\Delta E}{\Delta l_{x}}$ que é extraído dos espectros experimentais.

A soluçāo do problema nuclear se resume em resolver a equação de auto-valores:

$$
H^{\omega} \Psi^{\omega}=E^{\omega} \Psi^{\omega}
$$

onde $H^{\omega}$ é a hamiltoniana de "Cranking", $E^{\omega}$ é chamado de routhiano ou energia no referencial intrínseco do núcleo, e $\Psi^{\omega}$ é a funçāo de onda correspondente. A função de 
onda $(\Psi)$ e a hamiltoniana $(H)$ no referencial do laboratório se relacionam com $\Psi^{\omega}$ e $H_{o}$, do sistema intrínseco, através da transformação unitária:

$$
\Psi=R(\omega t) \Psi^{\omega}
$$

e

$$
H=R(\omega t) H_{o} R(\omega t)^{-1},
$$

onde $R(\omega t)$ é o operador de rotação:

$$
R(\omega t)=e^{-i \omega t J_{x} / \hbar}
$$

A hamiltoniana de "Cranking" é dada por:

$$
H^{\omega}=H_{o}-\omega J_{x}
$$

onde $H_{o}$ é a hamiltoniana do sistema intrínseco, $\omega$ é a freqūência angular de rotação neste referencial e $J_{x}$ é a projeção do operador momento angular no eixo de rotação, sendo o termo $\omega J_{x}$ correspondente à interação de Coriolis.

O tratamento de HFB da hamiltoniana $H^{\omega}$, que considera as correlações de emparelhamento, leva ao routhiano de quasi-partícula única:

$$
h_{q p}^{\omega}=h_{o}-\omega j_{x}-\Delta\left(P^{\dagger}+P\right) / 2-\lambda N,
$$

onde $h_{o}$ é a hamiltoniana do tipo Nilsson triaxial, ou seja hamiltoniana do oscilador harmônico modificado, e os operadores $P^{\dagger}$ e $P$ criam o campo médio de emparelhamento, com intensidade dada pelo parâmetro $\Delta$. O multiplicador de Lagrange $\lambda$ recupera o valor médio de $\mathrm{N}$, pois o número de partículas não é conservado.

Para reduzir a complexidade introduzida pelas correlaçōes de emparelhamento é utilizado aqui o formalismo de quasi-partícula. Os operadores $P^{\dagger}$ e $P$, que a princípio criam e aniquilam pares de partículas, são considerados operadores que misturam estados de partícula e buraco da quasi-partícula [14]. 
Os auto-valores de $h_{q p}^{\omega}$ sāo chamados routhianos de quasi-partícula $\left(e^{\omega}\right)$ :

$$
h_{q p}^{\omega} \psi^{\omega}=e^{\omega} \psi^{\omega}
$$

Tomando-se o valor médio da equação 2.6 obtemos:

$$
e^{\omega}=<\psi^{\omega}\left|h_{q p}^{\omega}\right| \psi^{\omega}>=e-\omega i_{x},
$$

com $i_{x}=<j_{x}>=-\frac{d e^{\omega}}{d \omega}$, que é chamado de momento angular alinhado, ou simplesmente alinhamento da quasi-partícula e

$$
e=<h_{o}-\Delta\left(P^{\dagger}+P\right) / 2-\lambda N>
$$

é o valor esperado da energia no sistema laboratório.

A hamiltoniana da equação 2.6 comuta com o operador de paridade $\Pi$ e com $R_{x}(\pi)$ que é a rotação de um ângulo $\pi \mathrm{em}$ torno do eixo de rotação $x$. As auto-funçōes $\psi^{\omega}$ podem ser classificadas por estas simetrias, resultando:

$$
\begin{aligned}
& \Pi \psi_{(\pi, \alpha)}=\pi \psi_{(\pi, \alpha)}, \quad \operatorname{com} \quad \pi= \pm 1 \\
& e^{-i \pi j_{x}} \psi_{(\pi, \alpha)}=e^{-i \pi \alpha} \psi_{(\pi, \alpha)}=r \psi_{(\pi, \alpha)}
\end{aligned}
$$

$\operatorname{com} r=-i(\alpha=1 / 2)$ e $+i(\alpha=-1 / 2)$, onde o novo número quântico $\alpha$ é chamado de "signature" e é uma quantidade aditiva.

Os estados de partícula única com valores de $\alpha= \pm 1 / 2$ são degenerados para $\omega=0$. Esta degenerescência é quebrada à medida que a rotação aumenta, onde a interação de Coriolis separa as componentes de "signature" diferentes. O valor em energia desta separação é chamada de "signature splitting" e a componente $\alpha$ de menor energia é dita favorecida e é dada pela relação $j=\alpha+2 n$, onde $j$ é o momento angular da partícula e $n$ é um número inteiro. 
Os valores de $\alpha= \pm 1$ são relativos aos spins ímpares $I=1,3,5 \ldots$ enquanto que $\alpha=0$ implica em spins pares $I=0,2,4 \ldots$, pois para uma banda rotacional de sequêencia $\Delta I=2$, com determinada "signature" temos $I=\alpha \bmod (2)$.

\subsubsection{Transformação dos Dados Experimentais para o Sistema Intrínseco}

Para a utilização do "Cranking Shell Model" é conveniente transformar os dados do esquema de decaimento para o sistema do referencial em rotação. Esta troca de coordenadas para o sistema intrínseco (sistema em rotação) permite a comparação direta entre os dados experimentais e teóricos, e também permite serem trabalhadas as características de partícula independente.

A energia de excitação no sistema laboratório $E(\omega)$ e no sistema em rotaçāo $E^{\prime}(\omega)$ são relacionadas por:

$$
E^{\prime}(\omega)=E(\omega)-\omega I_{x}
$$

sendo $\omega=-\frac{d E}{d I_{x}}$, construído a partir dos dados experimentais pela aproximação da fórmula:

$$
\hbar \omega(I)=\frac{E_{I+1}-E_{I-1}}{I_{x}(I+1)-1}
$$

onde é considerada uma seqüência $\Delta I=2$ de mesma "signature" de uma banda rotacional.

O routhiano experimental é calculado através de:

$$
E^{\prime}(I)=\frac{1}{2}\left(E_{I+1}+E_{I-1}\right)-\omega(I) I_{x}(I) .
$$

O alinhamento $I_{x}$, que é a projeçāo do momento angular total I no eixo de rotaçāo, é dado semiclassicamente pela relaçāo:

$$
I_{x}(I)=\sqrt{(I+1 / 2)^{2}-K^{2}}
$$

sendo K a projeção do momento angular total no eixo de simetria do núcleo e em geral é dado pela regra de Gallagher-Moszkowski [19], supondo que os spins do próton e do 
nêutron se alinhem paralelamente, isto é:

$$
\begin{aligned}
& K=\Omega_{\pi}+\Omega_{\nu} \quad \text { se } \Omega_{\pi}=\Lambda_{\pi} \pm 1 / 2 \text { e } \Omega_{\nu}=\Lambda_{\nu} \pm 1 / 2 \\
& K=\left|\Omega_{\pi}-\Omega_{\nu}\right| \text { se } \Omega_{\pi}=\Lambda_{\pi} \pm 1 / 2 \text { e } \Omega_{\nu}=\Lambda_{\nu} \mp 1 / 2 \text {. }
\end{aligned}
$$

A fim de podermos comparar os dados experimentais com a energia e o alinhamento da quasi-partícula, no sistema em rotação, é preciso subtrair das quantidades totais a contribuição coletiva das partículas (caroço), aqui chamada de configuraçāo de referência, isto é:

$$
e^{\prime}(\omega)=E^{\prime}(\omega)-E_{g}^{\prime}(\omega)
$$

$\mathbf{e}$

$$
i_{x}(\omega)=I_{x}(\omega)-I_{x g}(\omega)
$$

onde $E_{g}^{\prime}$ e $I_{x g}$ são o routhiano e o alinhamento da configuração de referência, que usualmente é conhecida como sendo a banda do caroço par-par, ou freqüentemente chamada de vácuo de quasi-partícula.

O alinhamento, $I_{x g}$, e a energia de referência, $E_{g}^{\prime}$, sāo dados pelas relaçōes de Harris introduzindo os parâmetros de momento de inércia $\mathcal{J}_{0}$ e $\mathcal{J}_{1}$ [20]:

$$
I_{x g}(\omega)=\omega \mathcal{J}_{0}+\omega^{3} \mathcal{J}_{1}
$$

E levando-se em conta a integração da fórmula:

$$
I_{x}=-\frac{d E^{\prime}}{d \omega}
$$

temos:

$$
E_{g}^{\prime}(\omega)=-\int I_{x g}(\omega) d \omega=-\frac{1}{2} \mathcal{J}_{0} \omega^{2}-\frac{1}{4} \mathcal{J}_{1} \omega^{4}+\frac{1}{8 \mathcal{J}_{0}},
$$

onde $\frac{1}{8 \mathcal{J}_{0}}$ é a constante de integração [14], ajustada para obter $E_{g}^{\prime}(I=0)=0$. 
Os parâmetros de Harris $\left(\mathcal{J}_{0}\right.$ e $\left.\mathcal{J}_{1}\right)$ são obtidos para cada uma das bandas em estudo através de um ajuste de $I_{x}(\omega)$ aos dados experimentais abaixo do primeiro cruzamento permitido. Desta forma, o termo $i_{x}$ da equação 2.17 é considerado um parâmetro constante, e seu valor é determinado através do ajuste da equação:

$$
I_{x}(\omega)=i_{x}-\mathcal{J}_{0} \omega-\mathcal{J}_{1} \omega^{3}
$$

Para estimar os efeitos da força de polarização $\gamma$ sobre a deformação nuclear é preciso calcular o valor da energia total no referencial intrínseco, ou seja, o routhiano total $\left(E^{\omega}\right)$. Os cálculos são feitos usando a contribuição fenomenológica do caroço par-par e os routhianos das quasi-partículas (próton e nêutron), seguindo o procedimento de Frauendorf e May [21]:

$$
E^{\omega}(\gamma, \omega)=\sum_{\mu} e_{\mu}^{\omega}(\gamma, \omega)-\frac{1}{2} \mathcal{J}(\gamma, \omega) \omega^{2}+\frac{1}{2} V_{p o} \cos (3 \gamma)
$$

Aqui o primeiro termo é simplesmente a soma sobre os orbitais de quasi-partícula simples $\left(e^{\omega}\right)$ ocupados, enquanto que o segundo termo considera a rotação coletiva do caroço nuclear, sendo $\mathcal{J}(\gamma, \omega)=\left(\mathcal{J}_{0}+\frac{1}{2} \omega^{2} \mathcal{J}_{1}\right) \frac{4}{3} \cos ^{2}\left(\gamma+30^{\circ}\right)$, que é o momento de inércia efetivo. O terceiro termo é a parametrização do potencial nuclear em função de gama [21], onde $V_{p o}$ é a diferença de energia potencial entre as formas prolata e oblata.

Os diversos parâmetros são ajustados de modo a proporcionar uma maior compatibilidade possível com os dados experimentais. Os parâmetros a serem determinados são:

\section{"Gap" de emparelhamento $\Delta$ :}

O potencial de emparelhamento representa o grau de interaçāo entre um par de quasipartículas e é geralmente escolhido como $\Delta \approx 135 / \mathrm{A}(\mathrm{MeV})$, que para esta regiāo de massa fica em torno de $1 \mathrm{MeV}$. 


\section{Parâmetros de Nilsson $\kappa$ e $\mu$ :}

Os valores adotados são constantes para cada camada $\mathrm{N}$ do oscilador [22]:

$$
\begin{aligned}
& \text { Para prótons: } \quad N=5 \quad \kappa=0.060 \text { e } \mu=0.65 \\
& N=4 \quad \kappa=0.065 \text { e } \mu=0.57 \\
& \text { Para nêutrons: } \quad N=5 \quad \kappa=0.062 \text { e } \mu=0.43 \\
& N=4 \quad \kappa=0.070 \text { e } \mu=0.39
\end{aligned}
$$

Parâmetro de deformação $\beta$ :

O parâmetro de deformação quadrupolar $\beta$ foi extraído das fórmulas empíricas [23] aplicadas aos valores conhecidos do primeiro estado excitado $2^{+}$dos núcleos par-par vizinhos, de acordo com o procedimento descrito na referência [24], onde o valor de $\beta$ é dado por:

$$
\beta=\sqrt{\frac{1224}{\bar{E}_{2^{+}} A^{7 / 3}}}
$$

onde $\bar{E}_{2^{+}}$é a energia média das primeiras transições $2^{+} \rightarrow 0^{+}$dos núcleos par-par vizinhos. Obteve-se o valor $\beta=0.14$ para o núcleo de ${ }^{138} \mathrm{Pr}$.

\section{Potencial químico $\lambda$ :}

Os valores das energias dos níveis de Fermi para prótons e nêutrons, $\lambda_{p}$ e $\lambda_{n}$, foram ajustados para recuperar o valor médio do número de partículas $\mathrm{Z}$ e $\mathrm{N}$, respectivamente; para o núcleo em estudo, os valores obtidos foram:

$$
\begin{aligned}
& \lambda_{p}=5.62 \hbar \omega_{0} \\
& \lambda_{n}=6.10 \hbar \omega_{0}
\end{aligned}
$$


onde $\hbar \omega_{o}=41 \cdot A^{-1 / 3}(\mathrm{MeV})$ que corresponde a $7.93 \mathrm{MeV}$ para o núcleo de ${ }^{138} \mathrm{Pr}$.

Parâmetros de Harris $\mathcal{J}_{O}$ e $\mathcal{J}_{1}$ :

Como mencionado anteriormente, os valores de $\mathcal{J}_{O}$ e $\mathcal{J}_{1}$ foram ajustados aos dados experimentais admitindo-se o alinhamento $\left(i_{x}\right)$ constante e os valores encontrados estão na seção 4.2, onde são apresentados os resultados dos cálculos das transformações dos dados experimentais para o sistema intrínseco.

Diferença de energia prolata-oblata $V_{p o}$ :

As previsões dos modelos teóricos baseados em potenciais de Woods-Saxon, Oscilador Modificado e folded-Yukawa [2], quanto ao valor da diferença de energia potencial entre as formas prolata e oblata, apresentam discrepâncias de até $1 \mathrm{MeV}$ entre si.

$\mathrm{Na}$ referência [21] os autores propōem que o valor de $\mathrm{V}_{\text {po }}^{\circ}$ seja ajustado de maneira que a diferença entre os mínimos das duas "signatures" reproduza o valor de $\Delta e^{\prime}$ experimental. Em alguns casos o valor de $V_{p o}$ é escolhido de acordo com a sistemática da região de massa, e para nós este valor de sistemática está em torno de $-0.4 \mathrm{MeV}$.

\subsection{Modelo de Camadas com Projeção de Momento An- gular}

Sabemos que o tratamento quântico de um sistema de muitos corpos é difícil devido ao grande número de graus de liberdade envolvido. E que para núcleos pesados, o espaço de configuraçāo no modelo de camadas, onde a hamiltoniana deve ser diagonalizada, é extremamente grande. Porém, como nāo estamos interessados numa descriçāo completa do sistema e se limitarmos nosso interesse para uma certa classe de estados, um cálculo do tipo 
de modelo de camadas pode ser feito usando-se um conjunto selecionado de configurações.

Esta é a principal característica desta teoria, que seleciona uma base de muitos corpos de acordo com a importância física dos estados, isto é, seleciona-se uma base contendo somente os estados essenciais à física que queremos descrever. Deste modo, o espaço de configuraçāo, no qual a hamiltoniana do sistema será diagonalizada, será bem menor em comparação com o modelo de camadas usual possibilitando a descrição de núcleos pesados.

Este modelo de camadas com projeção de momento angular tem sido aplicado com sucesso em núcleos par-par, ímpares e ímpar-ímpar na regiāo de terras raras $[15,25,26]$.

\subsubsection{Revisão da Teoria}

A descrição do sistema nuclear, neste modelo, é feita através de uma hamiltoniana esquemática dada por:

$$
\widehat{H}=\widehat{H}_{o}-\frac{\chi}{2} \sum_{\mu} \widehat{Q}_{\mu}^{\dagger} \widehat{Q}_{\mu}-G_{M} \widehat{P}^{\dagger} \widehat{P}-G_{Q} \sum_{\mu} \widehat{P}_{\mu}^{\dagger} \widehat{P}_{\mu}
$$

onde:

1o termo - $\widehat{H}_{o}$ é a hamiltoniana de camadas de partícula única (esférico), que contém o potencial químico $\lambda$.

$2^{\circ}$ termo - representa a interação quadrupolo-quadrupolo.

$3^{o}$ termo - corresponde à força de emparelhamento monopolar, e $G_{M}$ é a constante da força de emparelhamento, ajustada de tal modo a obter o "gap" de energia apropriado,

$$
\begin{aligned}
G_{M}=\left[G_{1} \pm G_{2} \frac{N-Z}{A}\right] / A \quad M e V & + \text { para prótons } \\
& - \text { para nêutrons }
\end{aligned}
$$

4 o termo - representa o emparelhamento quadrupolar, e supōe-se que $G_{Q}$ seja proporcional à força de emparelhamento monopolar $G_{M}$, tanto para prótons como para nêutrons. Esta constante de proporcionalidade é ajustada para os dados da região de massa em estudo e é dada por: 


$$
\left(\frac{G_{Q}}{G_{M}}\right)_{n}=\left(\frac{G_{Q}}{G_{M}}\right)_{p}=\alpha
$$

A força de emparelhamento quadrupolar é importante para reproduzir o ponto correto de cruzamento de bandas e atenuar a interação de Coriolis.

Antes de construirmos a base do modelo de camadas apropriada para a diagonalização, é necessário estabelecer a base das quasi-partículas. Isto é feito usando-se a representação de Nilsson + BCS, que gera orbitais de quasi-partículas deformados. A base de Nilsson é fixada pelos parâmetros de deformação do núcleo em questão, obtidos experimentalmente, ou através de estimativas dependentes de modelo. Neste contexto, a base de quasipartícula tem uma grande vantagem sobre a representação esférica, pois a maior parte das correlações (as de curto e longo alcance) são, em média, levadas em conta simultaneamente. Os estados da base são classificados pelo número de quasi-partículas $(0,2,4 \ldots$ para sistemas pares e 1,3,5... para ímpares), o qual definirá uma hierarquia natural das configurações. No entanto, esta base viola algumas simetrias do sistema original, como por exemplo a invariância rotacional e o número de partículas. Felizmente, estados com um bom momento angular podem ser restaurados com a ajuda do operador de projeção, enquanto o número de partículas é restaurado, em média, pelo multiplicador de Lagrange como usualmente é feito na teoria de BCS.

O operador de projeção do momento angular $\widehat{P}_{M K}^{I}$ é dado explicitamente pela expressāo [27]:

$$
\widehat{P}_{M K}^{I}=\frac{2 I+1}{8 \pi^{2}} \int d \Omega \mathbf{D}_{M K}^{I *}(\Omega) \widehat{R}(\Omega)
$$

onde $\widehat{R}(\Omega)$ é o operador de rotaçāo dado por:

$$
\widehat{R}(\Omega)=e^{i \alpha \widehat{J}_{x}} e^{i \beta \widehat{J}_{y}} e^{i \gamma \widehat{J}_{x}}
$$

sendo $\widehat{J}_{i}$ o operador de momento angular, $\mathbf{D}_{M K}^{I}(\Omega)$ a funçāo $\mathbf{D}, \Omega=(\alpha, \beta, \gamma)$ são os ângulos de Euler (onde $\alpha$ e $\gamma=[0,2 \pi]$ e $\beta=[0, \pi]$ ) e $d \Omega=d \alpha \operatorname{sen} \beta d \beta d \gamma$. O operador 
$\widehat{P}$ comuta com todos os operadores escalares (por exemplo a hamiltoniana) e tem as propriedades:

$$
\begin{gathered}
P_{K K^{\prime}}^{I \dagger}=\widehat{P}_{K^{\prime} K}^{I}, \\
\hat{P}_{K M}^{I} \hat{P}_{N K^{\prime}}^{J}=\widehat{P}_{K K^{\prime}}^{I} \delta_{I J} \delta_{M N} .
\end{gathered}
$$

Sendo assim, atuando sobre estados de quasi-partículas $\left|\phi_{k}\right\rangle$, o projetor gera um autoestado correspondente (não normalizado) com um bom momento angular I:

$$
\left|\Psi_{M K}^{I k}>=\widehat{P}_{M K}^{I}\right| \phi_{k}>
$$

onde $K$ é o número quântico magnético de $\left|\phi_{k}\right\rangle$. Deste modo, podemos construir a nova base do modelo de camadas, selecionando as configuraçōes fisicamente importantes e projetando-as em estados com bom momento angular. Podemos, ainda, nos referir a este método como "Projected Shell Model (PSM)".

Existem algumas vantagens desta aproximação em comparação com o modelo de camadas esférico. Inicialmente, a adição vetorial do momento angular, um procedimento conhecido no modelo de camadas usual, é feita automaticamente pelo projetor independentemente do número de partículas envolvido. Segundo, estados projetados construídos a partir de alguns estados de quasi-partículas, as quais por já incluirem informaçōes sobre correlaçōes criam uma hierarquia, fornecem uma base razoável. No modelo de camadas usual, no entanto, é necessário considerar todas as configuraçōes possíveis (nenhuma hierarquia é conhecida para sistemas deformados).

A base do modelo de camadas utilizada no presente trabalho é a seguinte:

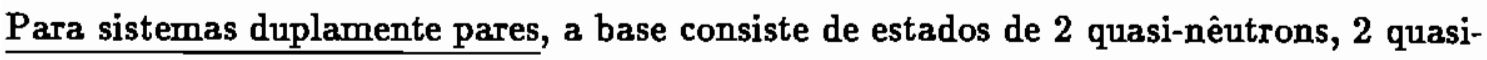
prótons e de 4 quasi-partículas (2 nêutrons e 2 prótons), projetados sobre o vácuo:

$$
\begin{array}{r}
\widehat{P}_{M 0}^{I} \mid 0>, \\
\widehat{P}_{M K_{\nu}}^{I} a_{\nu}^{\dagger} a_{\nu^{\prime}}^{\dagger} \mid 0>
\end{array}
$$




$$
\begin{array}{r}
\hat{P}_{M K_{\pi}}^{I} a_{\pi}^{\dagger} a_{\pi^{\prime}}^{\dagger} \mid 0>, \\
\widehat{P}_{M K_{\nu \pi}}^{I} a_{\nu}^{\dagger} a_{\nu}^{\dagger} a_{\pi}^{\dagger} a_{\pi^{\prime}}^{\dagger} \mid 0>,
\end{array}
$$

onde $K_{\nu}=K_{\nu}+K_{\nu^{\prime}}, K_{\pi}=K_{\pi}+K_{\pi^{\prime}}$ e $K_{\nu \pi}=K_{\nu}+K_{\pi}$ são as somas dos números quânticos $K$ das quasi-partículas em questão.

Para núcleos ímpar-ímpar a base é formada por estados projetados de 1 quasi-nêutron e 1 quasi-próton:

$$
\widehat{P}_{M K_{\nu \pi}}^{I} a_{\nu}^{\dagger} a_{\pi}^{\dagger} \mid 0>
$$

Para núcleos com $\mathrm{N}$ ímpar, a base é construída a partir de estados projetados de 1 quasipartícula e 3 quasi-partículas (1 quasi-nêutron e 2 quasi-prótons):

$$
\begin{array}{r}
\hat{P}_{M K_{\nu}}^{I} a_{\nu}^{\dagger} \mid 0>, \\
\widehat{P}_{M K_{\nu \pi}}^{I} a_{\nu}^{\dagger} a_{\pi}^{\dagger} a_{\pi^{\prime}}^{\dagger} \mid 0>
\end{array}
$$

finalmente para sistemas com Z ímpar:

$$
\begin{array}{r}
\widehat{P}_{M K_{\pi}}^{I} a_{\pi}^{\dagger} \mid 0>, \\
\widehat{P}_{M K_{\nu \pi}}^{I} a_{\nu}^{\dagger} a_{\nu^{\prime}}^{\dagger} a_{\pi}^{\dagger} \mid 0>
\end{array}
$$

Na notação acima, $a_{\nu}^{\dagger}\left(a_{\pi}^{\dagger}\right)$ é o operador de criação de um quasi-nêutron (próton), constrứdo na representação de Nilsson + BCS.

As bases mostradas nas equaçōes 2.32, 2.33, 2.34 e 2.35 contêm ainda muitos estados se forem considerados todos os níveis de Nilsson ativos. Por isso, os estados são escolhidos seguindo a hierarquia de excitaçōes de quasi-partículas. A vantagem desta representaçāo é que apenas alguns estados de quasi-partícula projetados são suficientes para descrever o núcleo.

Expandimos os auto-estados da hamiltoniana em nossa base através de: 


$$
\left|\Psi_{M}^{I}>=\sum_{k} f_{k} \widehat{P}_{M K}^{I}\right| \phi_{k}>
$$

A equação de autovalores nesta base (para um dado I) tem a forma:

$$
\sum_{k^{\prime}}\left(H_{k k^{\prime}}-E N_{k k^{\prime}}\right) f_{k^{\prime}}=0
$$

e a normalização é escolhida tal que:

$$
\sum_{k k^{\prime}} f_{k} N_{k k^{\prime}} f_{k^{\prime}}=1
$$

onde os elementos de matriz são definidos como:

$$
\begin{aligned}
& H_{k k^{\prime}}=<\phi_{k}\left|\hat{H} \widehat{P}_{K K^{\prime}}^{I}\right| \phi_{k^{\prime}}> \\
& N_{k k^{\prime}}=<\phi_{k}\left|\hat{P}_{K K^{\prime}}^{I}\right| \phi_{k^{\prime}}>
\end{aligned}
$$

Deve-se notar que, o uso da matriz $N_{k k^{\prime}}$ na equação 2.37 ao invés de $\delta_{k k^{\prime}}$ da equação de autovalores convencional, se deve à nāo ortogonalidade da base projetada.

Cada estado projetado em 2.31 pode representar uma banda rotacional. O primeiro, $\widehat{P}_{M K} \mid 0>$, por exemplo, representa a banda do estado fundamental de um sistema duplamente par, no qual todas as partículas estão emparelhadas. Os demais estados projetados representam várias bandas construídas a partir da excitação de par(es) quebrado(s), ou de quasi-partícula nāo emparelhada em um sistema de massa ímpar.

Deste modo, define-se a energia rotacional da banda $k$ por:

$$
E(I)=\frac{\left\langle\phi_{k}\left|\widehat{H} \widehat{P}_{K K}^{I}\right| \phi_{k}\right\rangle}{\left\langle\phi_{k}\left|\widehat{P}_{K K}^{I}\right| \phi_{k}\right\rangle}=\frac{H_{k k}}{N_{k k}} .
$$

Isto representa o valor esperado da hamiltoniana com respeito ao estado projetado $k$ da quasi-partícula. Chama-se de "diagrama de bandas" o diagrama no qual as energias rotacionais das várias bandas sāo plotadas como função do spin I. Nestes diagramas, as 
bandas correspondem a estados ainda não diagonalizados, e uma vez efetuada a diagonalização da hamiltoniana obtém-se a banda yrast para a configuração específica $N_{N}$ e $N_{P}$. Estes diagramas contêm informações valiosas como por exemplo cruzamento de bandas, inversão de "signature", etc., além de possibilitar a identificação das configurações mais importantes para uma banda, em funçāo do seu spin, como também o spin do cabeça de banda. No diagrama de bandas pode-se ainda observar o "zig-zag" das bandas com valores baixos de $\mathrm{K}$, cuja amplitude decresce rapidamente com o aumento de $K$. 


\section{Capítulo 3}

\section{Parte Experimental}

\subsection{Introdução}

Para o estudo dos estados excitados do núcleo ímpar-ímpar de ${ }^{138} P_{r}$ utilizou-se a técnica de espectroscopia $\gamma$ em linha e a reação de fusão-evaporação ${ }^{128} \mathrm{Te}\left({ }^{14} \mathrm{~N}, 4 n \gamma\right){ }^{138} \mathrm{Pr}$, que popula estados de altos spins e apresenta alguns canais de saída que competem entre si. Portanto, foram efetuados cálculos de seç̧ões de choque de formação dos principais canais abertos na reação ${ }^{128} \mathrm{Te}+{ }^{14} \mathrm{~N}$ utilizando o código PACE2S [28] - "Projection Angular Momentum Coupled Evaporation". Este código utiliza o método Monte Carlo para simular a reação nuclear de fusão-evaporação. Os resultados destes cálculos (figura ??) mostram que o canal de saída de interesse $\left({ }^{138} \mathrm{Pr}\right)$ é o mais intenso em relação aos outros canais, e que o segundo canal mais forte é é ${ }^{137} \operatorname{Pr}$ que é bem conhecido na literatura [8]. Os outros canais abertos possuem seç̧ão de choque desprezível em comparação com os dois primeiros.

No início deste trabalho as únicas informaçōes disponiveis sobre ${ }^{138} \mathrm{Pr}$ eram os decaimentos $\beta^{+}$do ${ }^{138} \mathrm{Nd}$ que populam estados de baixo spin, e o decaimento do estado fundamental do ${ }^{138} \operatorname{Pr}\left(I^{\pi}=1^{+}\right)$com meia vida de $1.4 m$, e de um estado isomérico de $I^{\pi}=7^{-}$com meia vida de $2.1 h$ para o núcleo filho de ${ }^{138} \mathrm{Ce}$. 


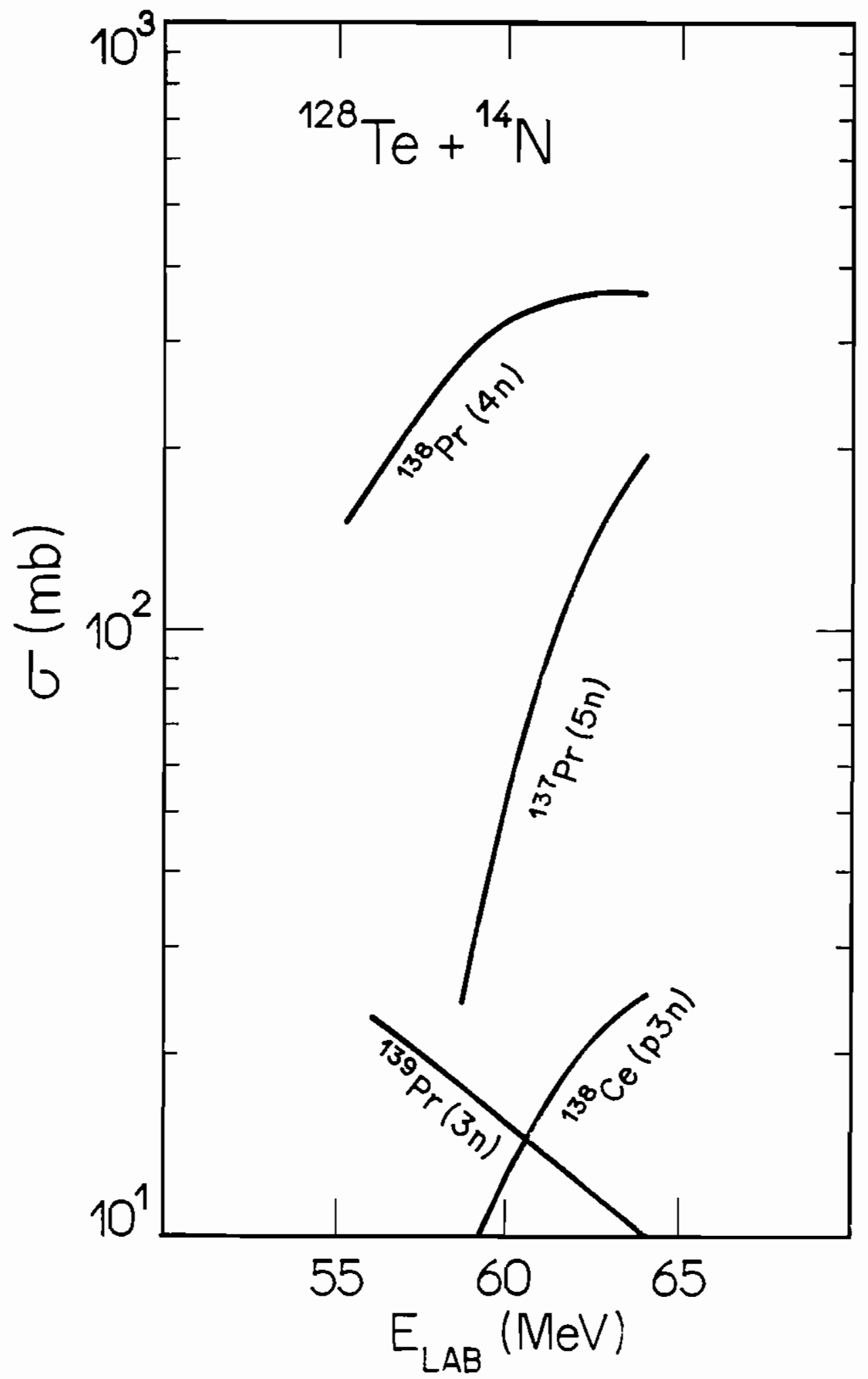

Figura 3.1: Cálculos da seç̧ão de choque de formação dos principais resíduos de evaporação, em funçāo da energia do feixe (Código PACE2S) para a reação ${ }^{128} \mathrm{Te}+{ }^{1 \cdot 1} \mathrm{~N}$. 


\subsection{Procedimento Experimental e Equipamentos}

No estudo do núcleo de ${ }^{138} \operatorname{Pr}$ foram realizadas medidas de função de excitação nas energias de 55,59 e $63 \mathrm{MeV}$, distribuiçōes angulares em $0^{\circ}, 65^{\circ}$ e $90^{\circ}$ e coincidências $\gamma-\gamma-t$ nas energias de 60 e $63 \mathrm{MeV}$ para a reaçāo ${ }^{128} \mathrm{Te}+{ }^{14} \mathrm{~N}$. O equipamento experimental utilizado para estas medidas constituiu-se essencialmente do acelerador Pelletron 8-UD (que incorpora a fonte de íons), câmaras de espalhamentos, alvos e detetores de radiação $\gamma$ do tipo HPGe (Hiper-Pure Germanium) e NaI, e também do sistema de aquisição de dados (CAMAC).

\subsubsection{Arranjo Experimental}

O feixe de íons negativos de $N^{-}$foi produzido pela fonte DUOPLASMATRON [29] que opera através de extração de íons de um plasma produzido por efeito termoiônico. O acelerador de partículas é do tipo "tandem" NEC, modelo 8-UD, desenvolvido para acelerar feixes de íons leves e pesados, de energias da ordem de até $4 \mathrm{MeV} /$ núcleon [30].

$\mathrm{O}$ alvo de ${ }^{128} \mathrm{Te}(99,2 \%)$, utilizado nas medidas foi doado pela Universidade de Munique e possui espessura de $\approx 930 \mu \mathrm{g} / \mathrm{cm}^{2}$, evaporado sobre um substrato de Au de $1 \mathrm{mg} / \mathrm{cm}^{2}$ e colocado sobre um suporte de aço inoxidável.

As primeiras medidas realizadas para o estudo do núcleo de ${ }^{138} \mathrm{Pr}$ foram as de coincidência $\gamma-\gamma-t$ da reaçāo ${ }^{128} \mathrm{Te}\left({ }^{14} \mathrm{~N}, 4 \mathrm{n} \gamma\right){ }^{138} \mathrm{Pr}$ com $E_{F}=60 \mathrm{MeV}$. Na figura 3.2 vê-se o desenho esquemático da câmara de espalhamento utilizada nestas medidas. Esta câmara é de alumínio tubular ( $36 \mathrm{~mm}$ de diâmetro e $1.5 \mathrm{~mm}$ de espessura) em forma de "T", onde os braços do "T" servem como entrada e saída do feixe, e o alvo permaneceu fixo no centro da câmara. A focalizaçāo do feixe foi feita com a utilizaçāo de um colimador de Ta (chapa com furo de $2 \mathrm{~mm}$ de diâmetro) preso a uma haste - isolada eletricamente da câmara - que é capaz de se deslocar verticalmente sem perda do vácuo. Este colimador 


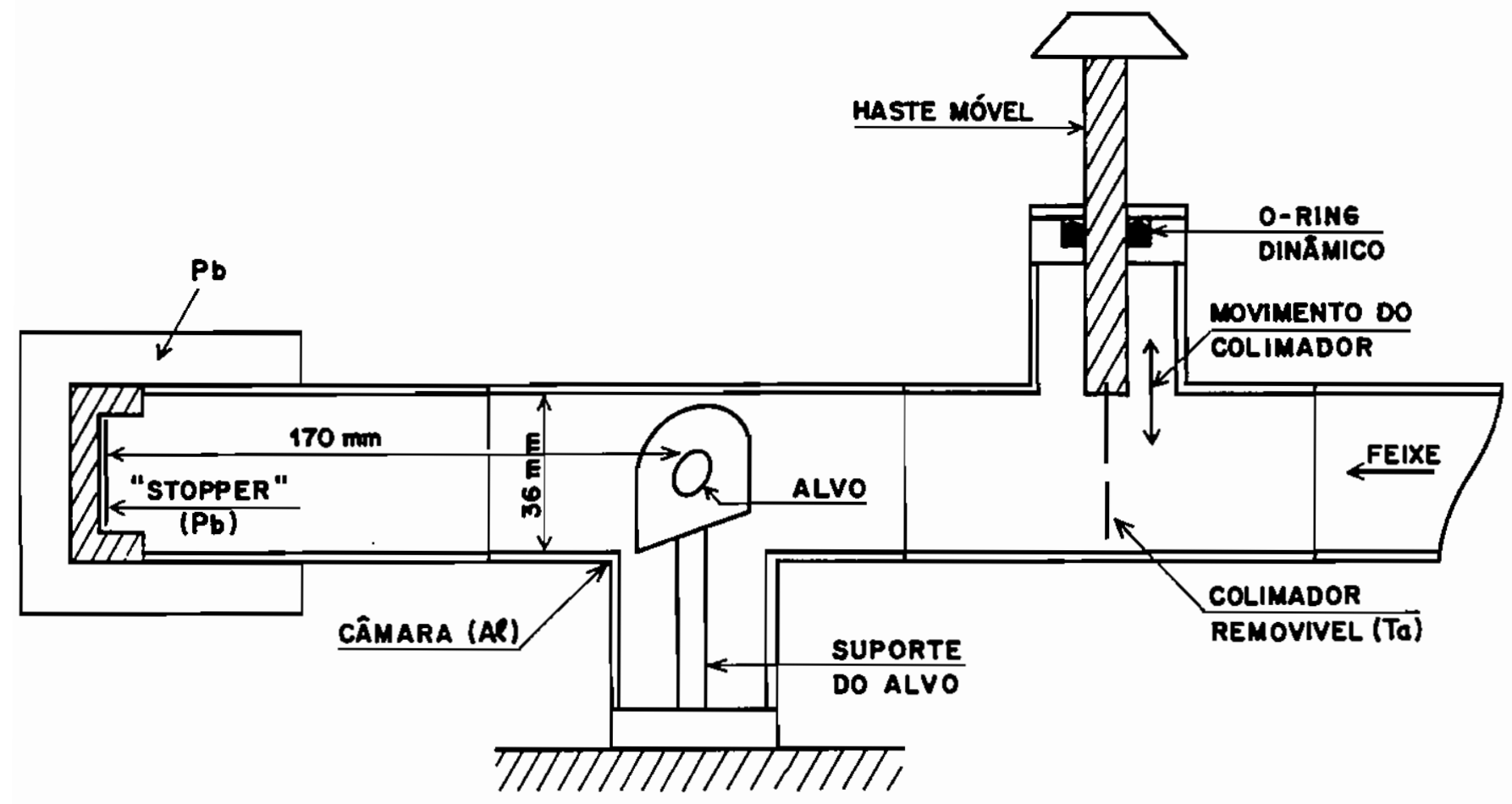

Figura 3.2: Desenho esquemático da câmara de espalhamento para as primeiras medidas de coincidência $\gamma-\gamma-t$.

encontrava-se em outra extensão "T" também alinhada com a câmara de espalhamento. Após a focalização do feixe, a haste foi deslocada para cima deixando livre a passagem do feixe evitando, deste modo, a produção de raios $\mathrm{X}$ provenientes do Ta. Utilizou-se neste experimento 3 detetores de germânio com resoluções da ordem de $2 \mathrm{keV}$ (figura 3.3).

Para as medidas de funçāo de excitaçāo $E_{F}=55,59$ e $63 \mathrm{MeV}$ e distribuiçāo angular em $0^{\circ}, 65^{\circ}$ e $90^{\circ}$ utilizou-se o alvo de ${ }^{128}$ Te a $45^{\circ}$ em relaçāo ao feixe, e 4 detetores, sendo 3 de germânio, de resoluçāo da ordem de $2.5 \mathrm{keV}$ fixos nos ângulos citados acima e distantes de aproximadamente $6 \mathrm{~cm}$ do alvo, e un detetor de $\mathrm{NaI}(\mathrm{Tl})$ (4" 4 ") disposto em cima da câmara de forma à cobrir o maior ângulo sólido possível $(\approx 2 \pi)$, minimizando deste modo 


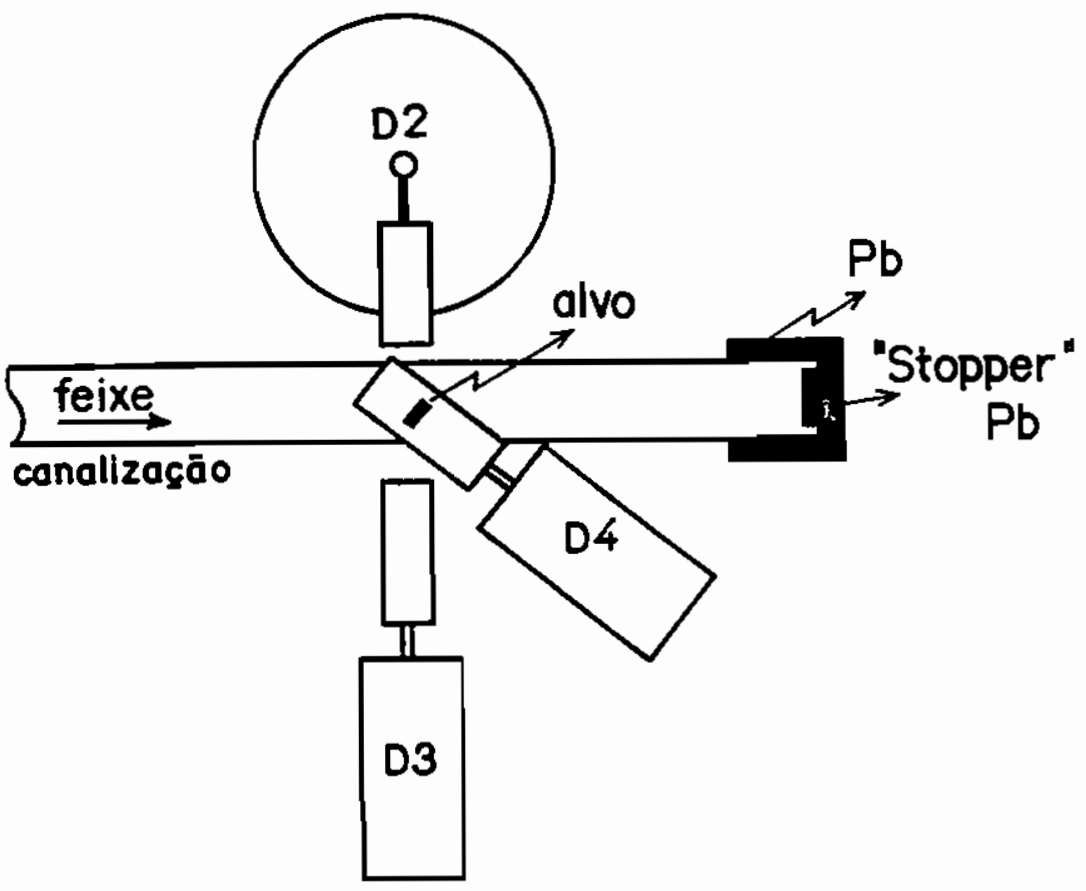

Figura 3.3: Montagem experimental dos detetores para as primeiras medidas de coincidência $\gamma-\gamma-t$.

as correlaçōes nas distribuições angulares. A finalidade do uso deste detetor de $\mathrm{NaI}(\mathrm{TI})$ foi a eliminaçāo de linhas provenientes de eventos de baixa multiplicidade, tais como gamas de decaimento radioativo, excitação coulombiana do alvo e gamas provenientes do fundo. Uma câmara de alumínio cilíndrica de $10 \mathrm{~cm}$ de diametro foi utilizada para estas medidas. Na tampa desta câmara foi colocado o suporte do alvo e um colimador de $\mathrm{Pb}$ utilizado na focalizaçāo do feixe. Os esquemas experimentais da disposiçāo dos detetores em relação ao feixe e da câmara utilizada na experiência são mostrados nas figuras 3.4 e 3.5 . Aproveitando-se a disposiçāo dos detetores, realizou-se também durante esta experiência medidas de coincidência $\gamma-\gamma-t$. 


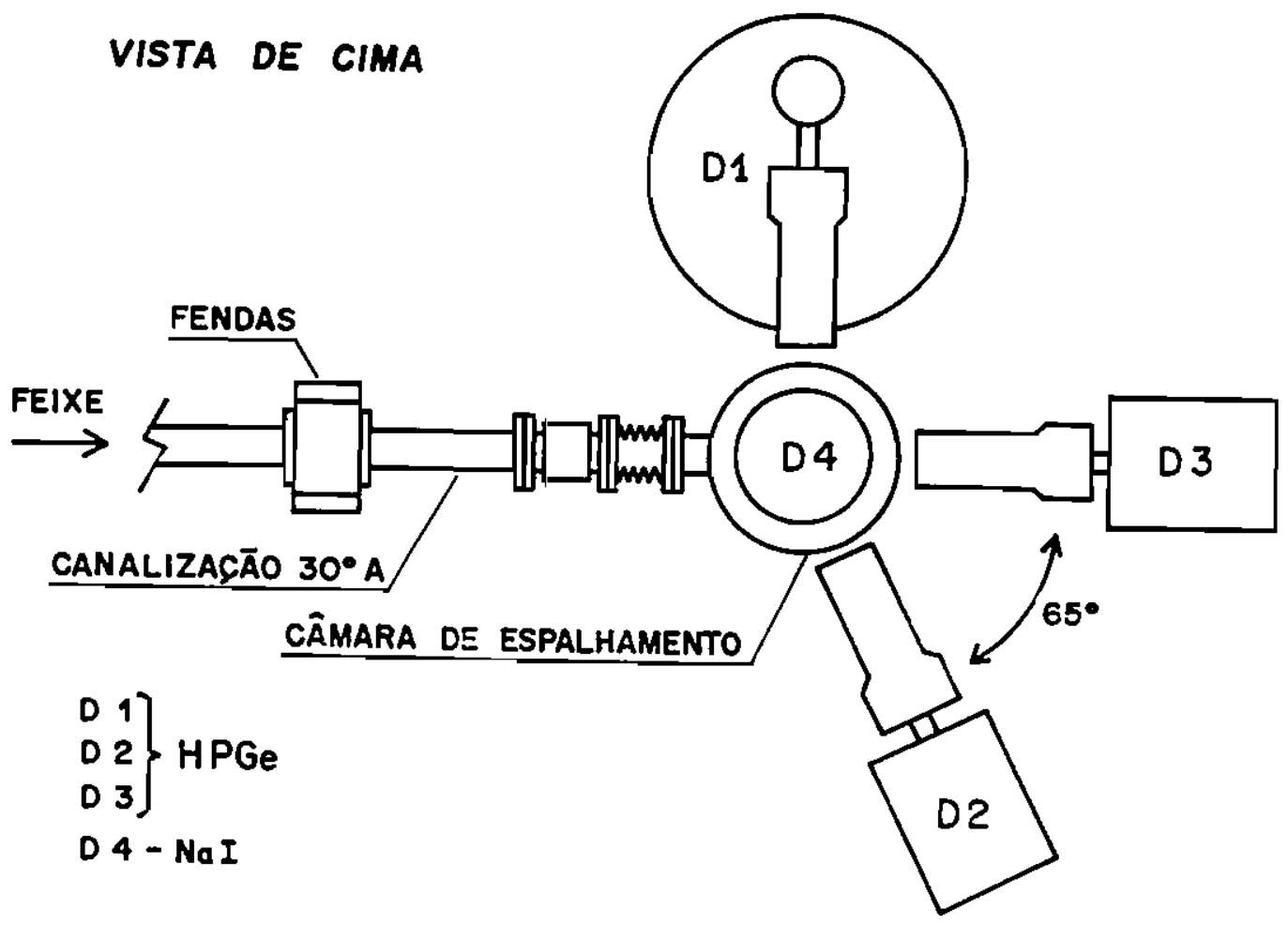

Figura 3.4: Montagem experimental dos detetores para medidas de distribuição angular, função de excitação (vista de cima).

A diferença entre este experimento e o anterior foi o acréscimo do detetor de $\mathrm{NaI}(\mathrm{Tl})$, que servia como marcador de tempo e filtro de multiplicidade. O objetivo desta nova experiência de coincidência $\gamma-\gamma-t$ foi melhorar a estatística em relação à anterior e confirmar os gamas candidatos ao núcleo de ${ }^{138} \mathrm{Pr}$.

Devido à baixa estatística obtida nas últimas experiéncias, realizou-se uma terceira medida de coincidência $\gamma-\gamma-t$ na energia de $E_{F}=63 \mathrm{MeV}$ e como resultado obteve-se o triplo da estatística da primeira tomada de dados de coincidência. Utilizou-se para esta última experiência a mesma câmara de alumínio em forma de " $T$ " da primeira medida de coincidência. Festa medida, utilizou-se o alvo de ${ }^{128} \mathrm{Te}$ a $90^{\circ}$ em relaçáo ao feixe e 5 


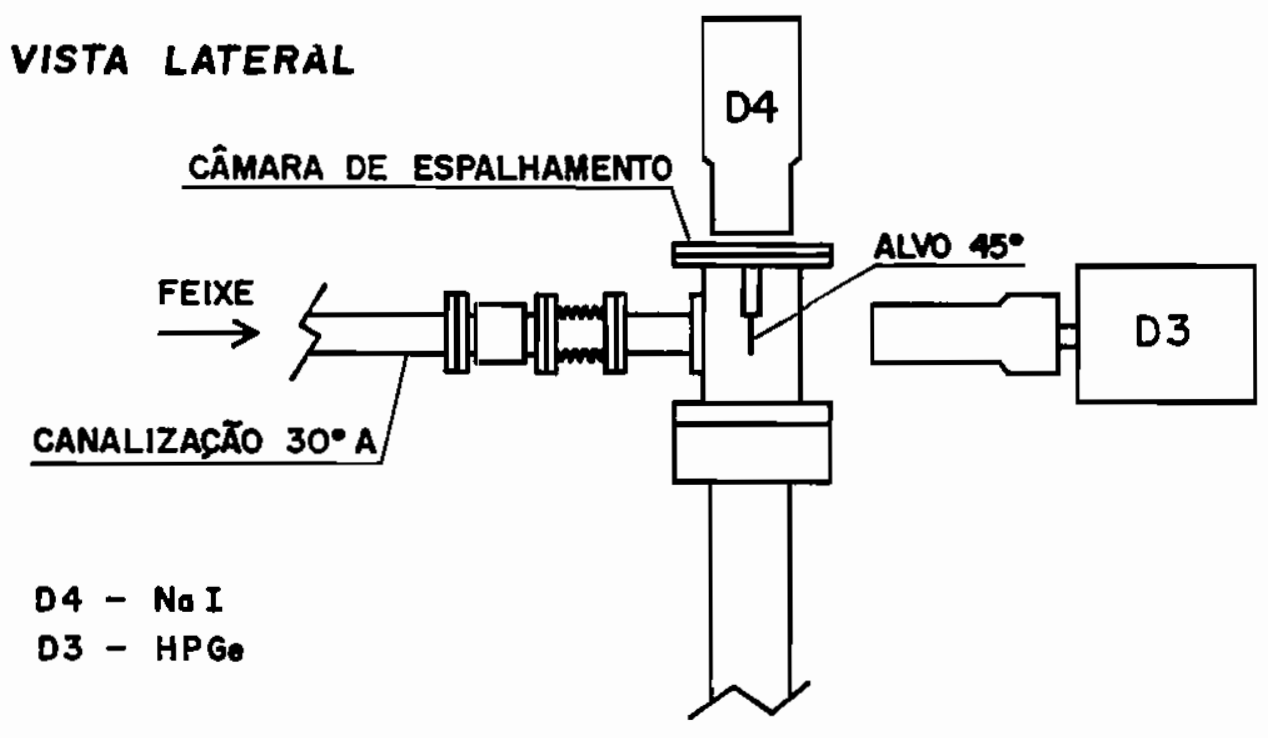

Figura 3.5: Montagem experimental dos detetores para medidas de distribuição angular, função de excitação (vista lateral).

detetores, sendo 4 de germânio de $\approx 2.5 \mathrm{keV}$ de resoiução distantes de aproximadamente $6 \mathrm{~cm}$ do alvo, e um detetor de $\mathrm{NaI}(\mathrm{Tl})$ (4"x4") disposto em cima da câmara a uma distância de aproximadamente $5 \mathrm{~cm}$ do alvo, figuras 3.6 e 3.7 .

\subsubsection{Eletrônica e Aquisição de Dados}

Para o controle, coleta e armazenagem dos dados obtidos nas várias medidas realizadas para o estudo da estrutura nuclear de ${ }^{138} \mathrm{Pr}$, utilizou-se o sistema de aquisição de dados do Laboratório Pelletron SPM-CAMAC [31], que utiliza a norma de interface de instrumentos CAMAC (Computer Automated Measurement and Control) [32]. Os módulos CAMAC são controlados pelo "Crate Controler" (CC) que é ligado ao computador Vax 11/780 através da interface "Brranch Driver" (Jorway 411). A forma de aquisiçāo é determinada 


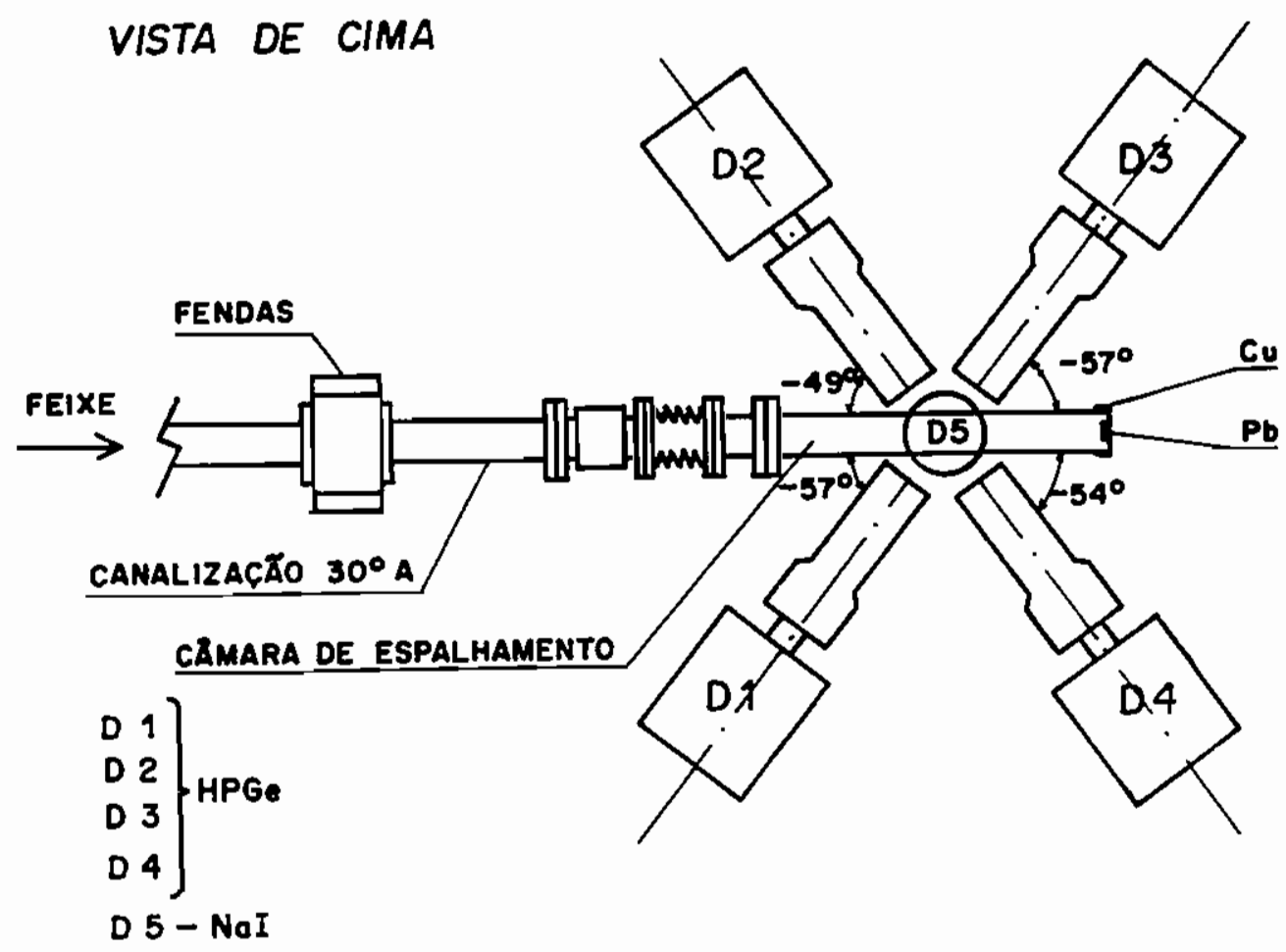

Figura 3.6: Montagem experimental dos detetores para medidas de coincidência $\gamma-\gamma-t$ (63 MeV) (vista de cima).

por um módulo controlador auxiliar chamado "EVENT HANDLER" (EH) que possui um microprocessador programável pelo experimentador.

No tratamento dos pulsos dos detetores de raios $\gamma$ de todas as medidas descritas acima utilizou-se uma eletrônica convencional de coincidência $\gamma-\gamma-t$ conforme figura 3.8. Para cada experiência em específico alterou-se o número de detetores de Ge bem como acrescentou-se em algumas delas um detetor de $\mathrm{NaI}(\mathrm{Tl})$ de 4 " $x 4$ " que servia como um marcador de tempo, sendo a coincidência feita através deste detetor com pelo menos um detetor de Ge.

O sinal linear (energia) de cada detetor de raios $\gamma$ é enviado a um Amplificador Linear (LA) que, associado a um Formador Linear de Pulsos (LGS), gera um pulso de altura proporcional à energia do gama, que será analisado pelo Conversor Analógico Digital (ADC). 


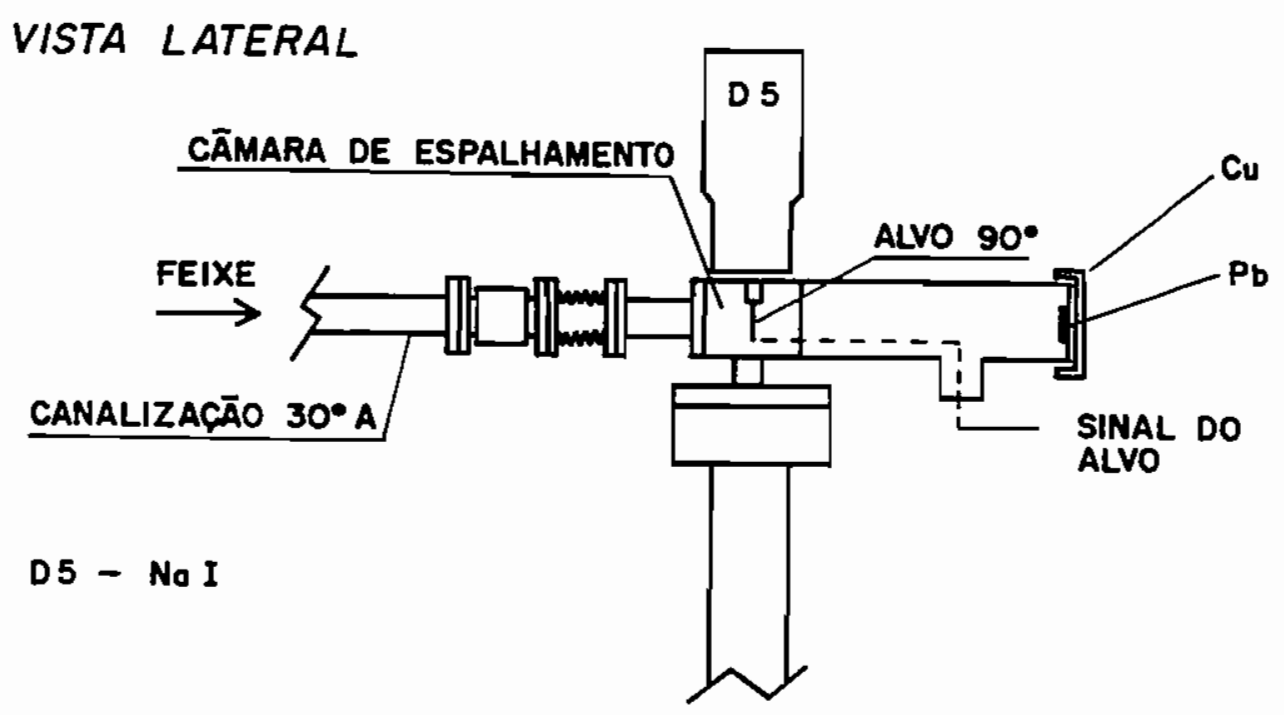

Figura 3.7: Montagem experimental dos detetores para medidas de coincidência $\gamma-\gamma-t$ $(63 \mathrm{MeV})$ (vista de lateral).

Os pulsos dos detetores $\gamma$ sāo também amplificados e diferenciados por um "Timing Filter Amplifier" (TFA) e introduzidos em um Discriminador de Frações Constantes (CFD), que gera pulsos rápidos que correspondem ao instante da detecção do raio $\gamma$. Estes pulsos são devidamente atrasados para que os diferentes detetores, que estão correlacionados, estejam coincidentes em tempo.

Estes pulsos rápidos provenientes do CFD dos detetores de raios $\gamma$ são introduzidos numa caixa lógica, que é ajustada para realizar as operaçōes lógicas necessárias de modo a fornecer um pulso de saída que indica a coincidência de no mínimo um detetor de Ge e $\mathrm{NaI}(\mathrm{Tl})$ (evento). A seguir, o pulso de saída da caixa lógica é enviado a uma entrada "start" do Conversor de Tempo Digital (TDC) e o mesmo pulso atrasado por um GDG (Gerador de Atrasos) avisa o sistema SPM-CAMAC da ocorrência de um evento. Um 


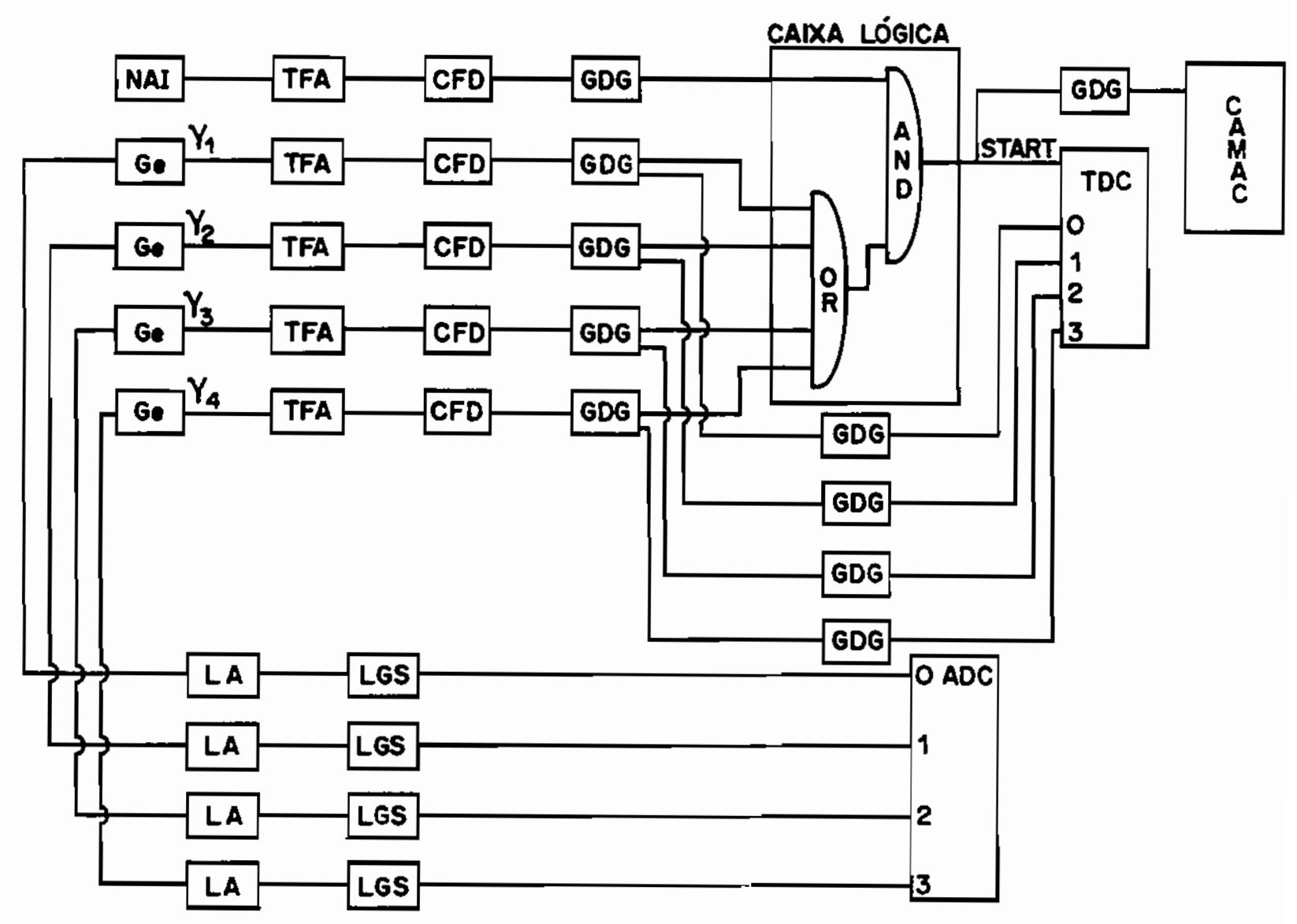

Figura 3.8: Exemplo do desenho esquemático do circuito eletrônico utilizado para medidas de coincidências $\gamma-\gamma-t(63 \mathrm{MeV})$.

pulso do CFD (detetor 1, 2, 3 ou 4) é introduzido em um GDG respectivo e depois na entrada "stop" do TDC, e a cada par de sinais de "start" e "stop" temos o intervalo de tempo entre os eventos. Este pulso final é o resultado da coincidência de três gamas, dois deles nos detetores de $\mathrm{Ge}$ e um no $\mathrm{NaI}(\mathrm{Tl})$. Esta foi a condição mínima para o evento de coincidência ser registrado. 
Ao receber um pulso externo, o EH reconhece a ocorrência de um evento, e não havendo nenhum impedimento (por exemplo uma condição de "wait" ou "stop"), executa o seu programa. No nosso caso ele zerava e fazia a leitura dos ADCs e TDCs, escrevia os parâmetros lidos em outro módulo CAMAC, um "buffer" de memória FIFO ("First In First Out"), que é na realidade a memória de dados do EH. Este "buffer" é programado para enviar um sinal de atenção "Look at Me" (LAM) ao CC quando tiver completado $3 / 4$ de sua capacidade de memória. O sistema de aquisição está preparado para receber uma interrupção associada a este LAM, quando entāo esvazia a memória FIFO e apaga o sinal de LAM.

O sistema de aquisição de dados do laboratório Pelletron possui vários processos concorrentes entre si, e estes são comandados pelo processo GERENTE que controla de um modo geral a aquisição, como por exemplo carregar os subprocessos (IO, SCAN4 e SVFШA), estabelecer as áreas de comunicação entre eles, bem como receber e distribuir os comandos para os vários subprocessos.

O subprocesso CAMAC-IO é o responsável pela comunicação do computador com o CAMAC; ele inicializa o sistema, reserva as áreas de memória, carrega o programa do EH, executa as operaçōes CAMAC para ler e zerar o FIFO, e controla o início e o final da aquisiçāo de dados. O subprocesso CAMAC-SCAN4 histograma os eventos escolhidos pelo experimentador para monitoração da aquisição, por exemplo espectros de energia ou tempo de um determinado detetor. Finalmente, o subprocesso CAMAC-SVFШA salva os eventos em arquivos em disco, agrupados em blocos de 8192 bytes, que posteriormente são gravados em fitas magnéticas de alta densidade para serem analisados "off-line". 


\subsection{Redução dos Dados Experimentais}

\subsubsection{Função de Excitação e Distribuição Angular}

As medidas de função de excitação e distribuição angular foram realizadas simultaneamente nas energias de $E_{F}=55,59$ e $63 \mathrm{MeV}$ com os três detetores de Ge fixos em $0^{\circ}, 65^{\circ}$ e $90^{\circ}$ e um $\mathrm{NaI}(\mathrm{TI})$ em cima da câmara de espalhamento (figuras 3.4 e 3.5). Os dados foram obtidos através da coincidência do detetor de $\mathrm{NaI}(\mathrm{Tl})$ com qualquer um dos detetores de germânio. $\mathrm{Na}$ análise destes dados utilizou-se o pacote de códigos computacionais VAXPAK [33] para ajuste das áreas dos picos e seus centróides (gaussianas com caudas exponenciais).

Para as medidas dos espectros de eficiência dos detetores utilizou-se a fonte de ${ }^{152} \mathrm{Eu}$ disposta na posição do alvo. As curvas de eficiência correspondentes a cada detetor foram obtidas calculando-se a eficiência relativa que é a razão entre a área ajustada, nos espectros de eficiência, e a intensidade relativa de cada gama [34].

Foram obtidos espectros de eficiência "singles" e de coincidência relativa entre os detetores, onde o primeiro é obtido sem passar pelo circuito de coincidência, ou seja, retira-se os sinais diretamente dos amplificadores lineares (LA) (figura 3.8); já no segundo caso os pulsos passam pelo circuito de coincidência, levando-se em conta, deste modo, o efeito do CFD (Constant Fraction Discriminator) sobre os gamas de baixa energia durante o processo de aquisiçāo de dados. Este fato é considerado porque os dados foram adquiridos considerando-se a coincidência do detetor de $\mathrm{NaI}(\mathrm{Tl})$ com pelo menos um detetore de Ge. Adotou-se para um determinado detetor a eficiência igual a um e normalizou-se os outros 2 para este detetor, já que os detetores utilizados sāo bastante semelhantes. A função ajustada que mostra a relação de eficiência destes dois detetores é apresentada na figura 3.9. A figura 3.10 mostra uma curva típica de eficiência "singles" de um dos detetores utilizado na experiência de função de excitaçāo e distribuição angular. 


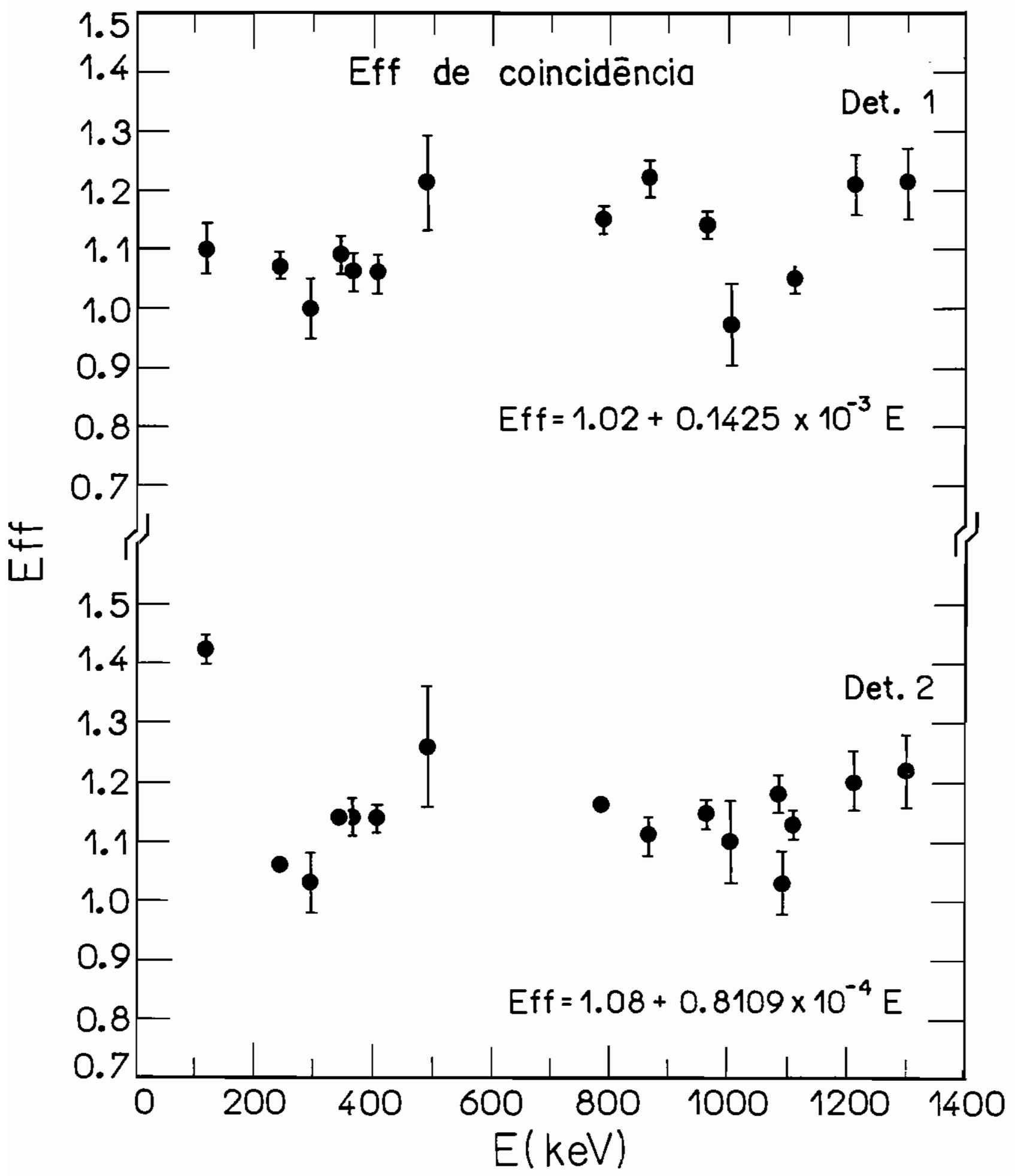

Figura 3.9: Eficiència de coincidência relativa entre os detetores utilizados na experiência de funçāo de excitação e distribuiçāo angular. 


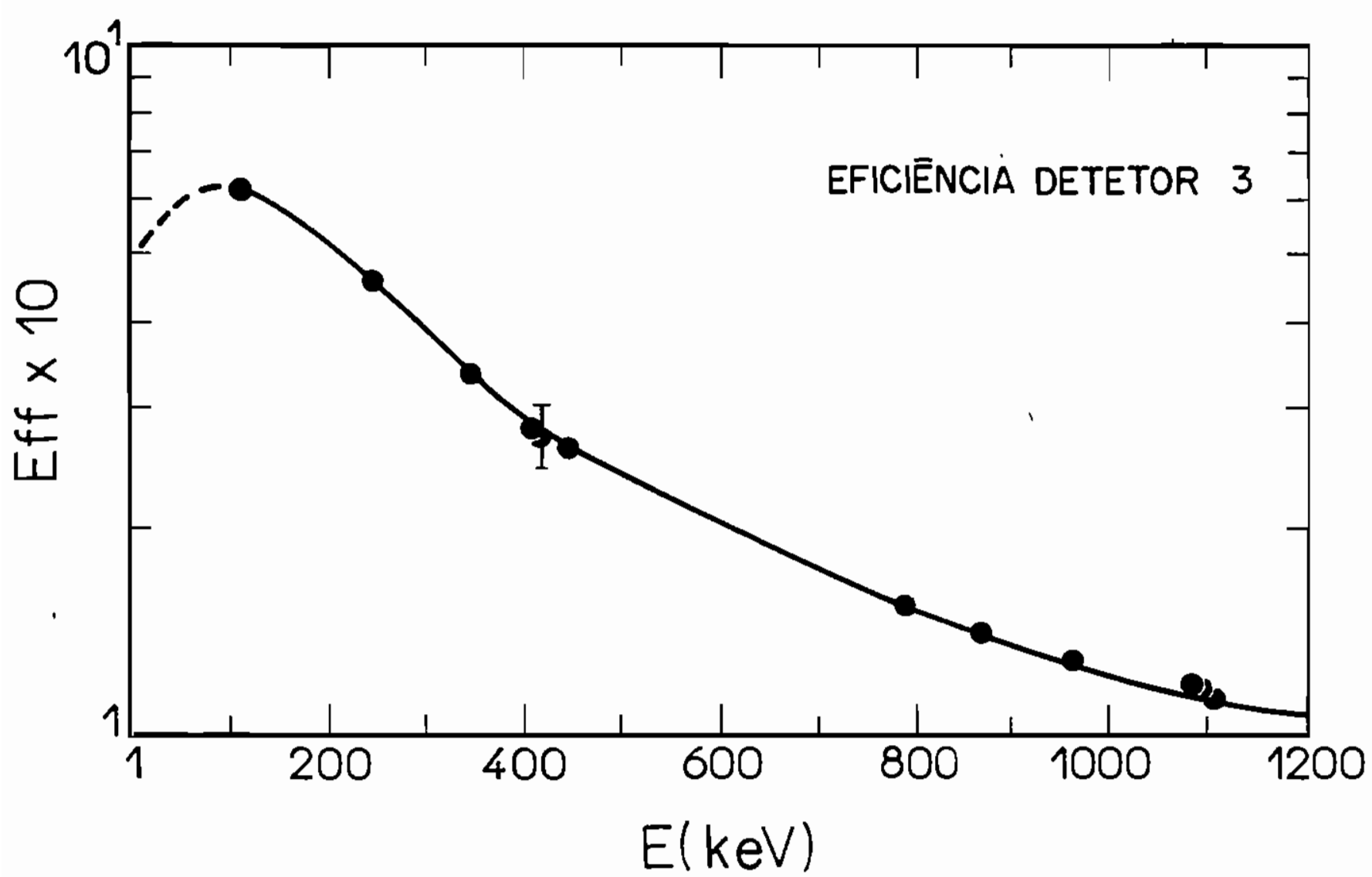

Figura 3.10: Curva típica de eficiência "singles" do detetor 3, utilizado na experiência de função de excitação e distribuição angular. Os pontos já incỉuem os erros nas medidas.

\section{a) Função de Excitação:}

Os dados de função de excitação foram tomados em $65^{\circ}$ a fim de minimizar efeitos de diferentes polaridades. As curvas de função de excitação foram plotadas normalizando os gamas à energia mais alta de $63 \mathrm{MeV}$ (figura 3.11), onde as transiçōes correspondentes aos canais de saída mais intensos da reação, tais como ${ }^{138} \operatorname{Pr},{ }^{139} \operatorname{Pr}$ e ${ }^{137} \mathrm{Pr}$, tendem a se acurnular em torno de curvas semelhantes, mostrando razoável acordo com as previsōes do código PACE2S (figura 3.1). Na figura 3.12 estes dados sāo normaijzados pela intensidade 
da transição de $136 \mathrm{keV}$, permitindo através desta normalização distinguir as transiçōes de spins mais altos $(402,396,187,252 \mathrm{keV}$, etc. $)$ das de mais baixos $(515,199$ e $694 \mathrm{kev})$. Estes dois critérios de normalização foram úteis na identificação e no posicionamento dos gamas no esquema de níveis.

\section{b) Distribuição angular:}

As estimativas dos valores das intensidades das distribuições angulares foram calculadas através da relação $\mathrm{I}(\theta)=\mathrm{A} / \mathrm{C} \cdot E_{f f}$, onde $\mathrm{A}$ é a área dos picos de raios gama (espectros de energia de $63 \mathrm{MeV}$ ), $\mathrm{C}$ é uma constante de normalização dos três detetores e $E_{f f}$ é a eficiência relativa entre os detetores.

A função que descreve a dependência angular das coincidências $\gamma-\gamma$ é dada por:

$$
W(\theta)=a_{0}+a_{2} P_{2}(\theta)+a_{4} P_{4}(\theta)
$$

onde $P_{2}$ e $P_{4}$ são os polinômios de Legendre pares que aparecem devido à conservação da paridade na interação eletromagnética. Os coeficientes $a_{0}, a_{2}$ e $a_{4}$ sāo os parâmetros ajustados e $\theta$ é o ângulo do detetor em relação ao feixe.

As razões $a_{2} / a_{0}$ e $a_{4} / a_{0}$, calculadas para as várias transiçōes gama, sāo apresentadas nas tabelas 3.1 e 3.2. Dos resultados das distribuições angulares nota-se que a maioria das transições observadas no núcleo de ${ }^{138} \operatorname{Pr}$ possuem multipolaridade dipolar $(136,199,396$, $402,515,545 \mathrm{keV}$, etc.) e que somente alguns gamas possuem multipolaridade quadrupolar. Algumas curvas típicas de distribuição angular de gamas pertencentes ao núcleo de ${ }^{138} \mathrm{Pr}$ são apresentadas na figura 3.13 . 


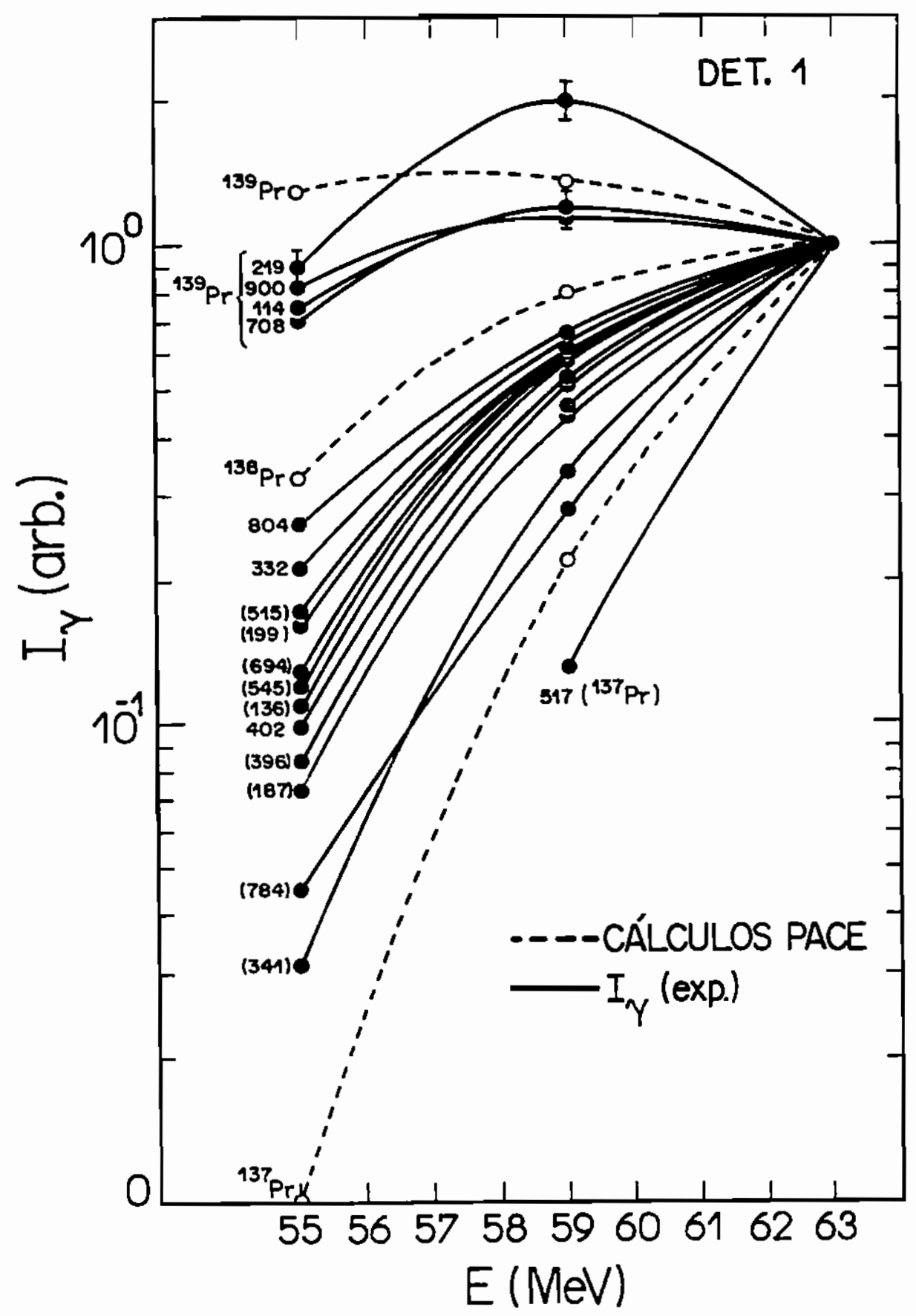

Figura 3.11: Funçāo de excitação das transiçōes gama da reação ${ }^{128} \mathrm{Te}\left({ }^{14} \mathrm{~N}, \mathrm{xn} \gamma\right)$. As energias das transiçōes são indicadas em keV. As curvas tracejadas correspondem à seç̧ão de choque dos resíauos de evaporação calculados através ảo código PACE2S. 


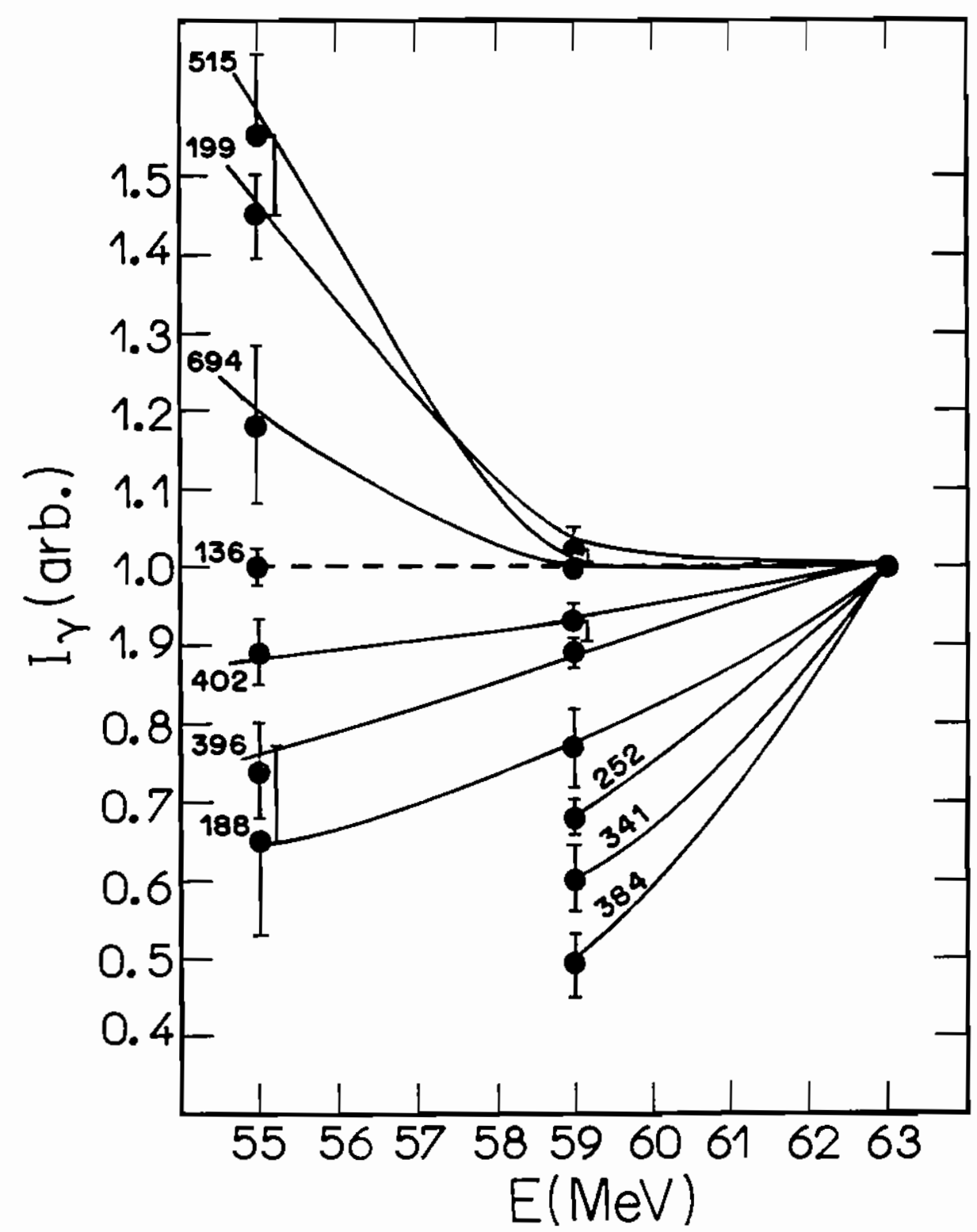

Figura 3.12: Funçāo de Excitaçāo das transições gama da reação ${ }^{128} \mathrm{Te}\left({ }^{14} \mathrm{~N}, 4 \mathrm{n} \gamma\right){ }^{138} \mathrm{Pr}$ normalizados pela transiçāo de $136 \mathrm{keV}$. Os gamas de 515, 199 e $694 \mathrm{keV}$ correspondem a baixo spin, enquanto os gamas de 136, 402, 396, 187, 252, 341 e $384 \mathrm{keV}$ são de alto spin. 


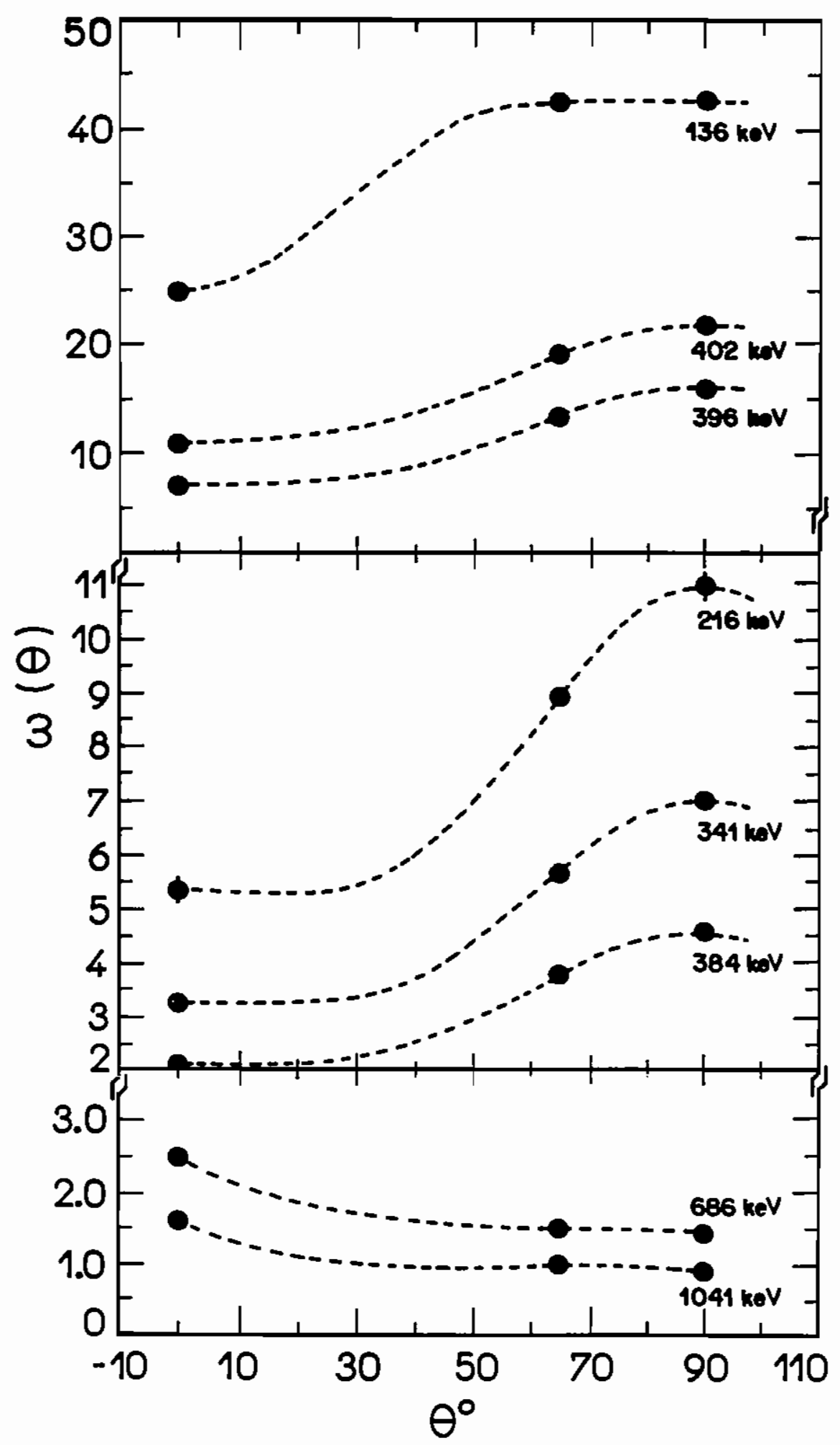

Figura 3.13: Curvas típicas de distribuição angular de gamas relevantes do núcleo de ${ }^{138} \mathrm{Pr}$. 


\subsubsection{Coincidência $\gamma-\gamma-t$}

Para a identificação dos gamas e construção do esquema de níveis do núcleo ímparimpar de ${ }^{138} \mathrm{Pr}$, foram realizadas além das medidas de funçāo de excitação e distribuiçāo angular, três medidas de coincidência $\gamma-\gamma-t$.

As primeiras medidas foram feitas com $E_{F}=60 \mathrm{MeV}$, onde se obtem a menor contribuição do segundo canal aberto na reaçāo $\left({ }^{137} \mathrm{Pr}\right)$; contudo apenas gamas com spin relativamente baixo foram observados. Nesta experiência, no entanto, observou-se ainda uma grande contaminaçāo de ${ }^{16} \mathrm{O}$.

A segunda experiência de coincidência $\gamma-\gamma-t$ foi realizada juntamente com as medidas de funçāo de excitação e distribuição angular aproveitando-se a disposiçāo dos 3 detetores de Ge e do detetor de $\mathrm{NaI}(\mathrm{Tl})$. Os dados obtidos em cada "run" de energia diferente (55, 59 e $63 \mathrm{Mev}$ ) foram somados para aumentar a estatística destas medidas; todavia estes dados obtidos nāo foram suficientes para sanar as dúvidas existentes na construçāo do esquema de níveis.

$\mathrm{Na}$ terceira e última experiência de coincidência $\gamma-\gamma-t$ com o feixe de ${ }^{14} \mathrm{~N}$ de energia incidente de $63 \mathrm{MeV}$ e com a utilização de 4 detetores de Ge, foi possível obter o triplo da estatística da primeira experiência. Como a população dos estados de spins altos é função da energia do feixe, procurou-se trabalhar, nesta experiência, com a energia máxima do acelerador (63 Mev para o ${ }^{14} \mathrm{~N}$ ), apesar da contaminaçāo do ${ }^{13 \tau} \operatorname{Pr}$ (segundo canal aberto na reação) que é muito bem conhecido na literatura. De um modo geral, com esta última experiência foi possível resolver as dúvidas existentes e construir o esquema de níveis final do ${ }^{138} \mathrm{Pr}$.

A aquisição dos dados de todas estas experiências de coincidência $\gamma-\gamma-t$ da reação ${ }^{128} \mathrm{Te}\left({ }^{14} \mathrm{~N}, 4 \mathrm{n} \gamma\right){ }^{138} \mathrm{Pr}$ foi feita em modo evento-por-evento e os dados foram guardados em forma bruta em fitas magnéticas para posterior análise. O processamento destes dacos em modo fila foi feito através do programa SCAN4 [33], cuja função é ordenar ("sorting") os eventos em histogramas uni- ou bidimensionais. As instruçōes para construção dos histogramas são escritas em linguagem CHIL, que compiladas produzem a lista de instruçōes (MIL) processadas pelo SCAN. 
No processamento dos dados ajustou-se o ganho dos diferentes detetores para, deste modo, somar os dados e melhorar a estatística. Os dados de cada experiência foram histogramados em matrizes biparamétricas: a) de eventos reais + casuais (total), b) de eventos casuais e c) uma matriz gama $x$ tempo para verificação de possíveis estados isoméricos. A projeção unidimensional da matriz biparamétrica obtida na última experiência de coincidência $\gamma-\gamma-t(63 \mathrm{Mev})$ é mostrada na figura 3.14 .

A escolha dos primeiros gamas candidatos de ${ }^{138} \mathrm{Pr}$ foi feita através da análise dos espectros de projeção total da matriz simetrizada e dos espectros simples da primeira experiência de coincidência $\gamma-\gamma-t(60 \mathrm{MeV})$. Eliminou-se destes espectros os gamas contaminantes provenientes da fusão do feixe com elementos contaminantes presentes no alvo (C e 0 ), e também outros gamas esperados na reação como: decaimento de ${ }^{138} \mathrm{Pr}$ para o ${ }^{138} \mathrm{Ce}$, gamas dos outros canais abertos na reação $\left({ }^{137} \mathrm{Pr},{ }^{139} \mathrm{Pr}\right.$ e $\left.{ }^{138} \mathrm{Ce}\right)$, bem como seus respectivos decaimentos, gamas de excitação coulombiana do núcleo alvo ${ }^{128} \mathrm{Te}$ e do "backing" de Au; aniquilação $\beta^{-} \beta^{+}(511 \mathrm{keV})$ e os gamas de radiação de fundo (espectro simples).

Analisou-se também a matriz de coincidência $\gamma-t$ para a verificação de um possível estado isomérico. As janelas nas energias dos gamas geram espectros de tempo, onde o pico observado corresponde aos eventos de coincidência verdadeira e a rampa (eventos atrasados) indica o decaimento do estado isomérico, e a linha de base ou fundo, representa os eventos casuais. A figura 3.15 mostra a projeção total desta matriz $(\gamma-t)$. A análise desta matriz não evidenciou nenhuma transição gama que possua meia vida longa, isto é, no intervalo de $20 n s$ até $1 \mu$ s. Acredita-se, no entanto, que possam haver no núcleo de ${ }^{138} \mathrm{Pr}$ estados com meia vida menor que $20 \mathrm{~ns}$ ou até mesmo maiores que $1 \mu \mathrm{s}$, porém há necessidade de se utilizar outras técnicas para medir estes valores.

A análise dos gamas coincidentes foi feita através do programa PANORAMIX [35] conforme descriçāo abaixo. 


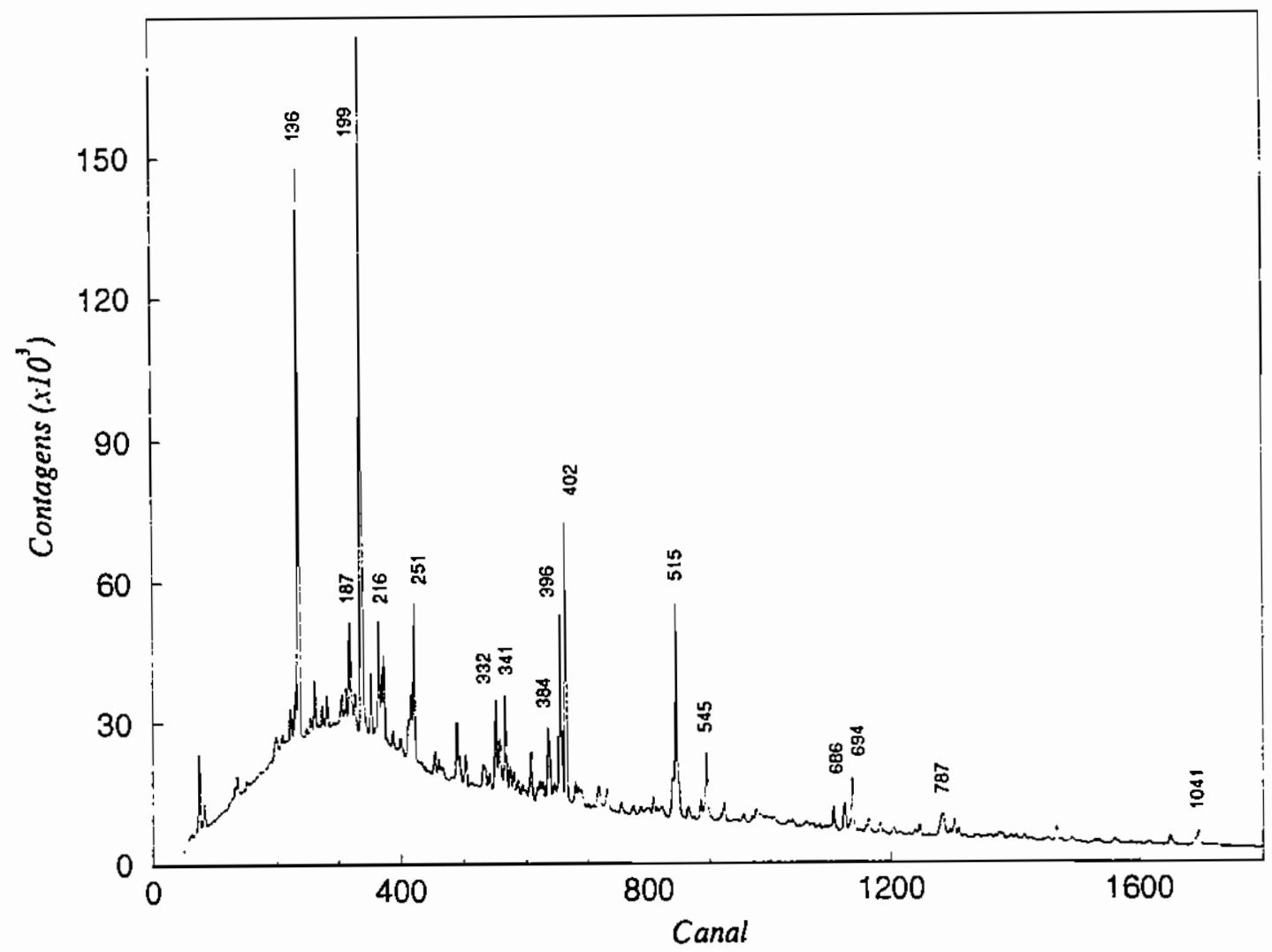

Figura 3.14: Espectro de projeção total da matriz simetrizada da experiência de coincidência $\gamma-\gamma-t(63 \mathrm{Mev})$ da reação $\left.{ }^{128} \mathrm{Te}\left({ }^{14} \mathrm{~N}, 4 \mathrm{n} \gamma\right)\right)^{138} \mathrm{Pr}$.

\subsubsection{Código Panoramix}

PANORAMIX é um programa iterativo de análise de matrizes bidimensionais de coincidéncias $\gamma-\gamma$ de um núcleo [35]. Os espectros de gamas coincidentes são obtidos através de janelas definidas a partir do espectro de projeção total, normalizados de acordo com a porcentagem da área do fotopico envolvido na construçāo da janela e com o respectivo fundo Compton subtraído. Como os espectros são em geral complexos, o código PANORAMIX permite estimar a contaminação dos picos a serem janelados e então pode-se efetuar uma descontaminação da janela, subtraindo do pico em estudo um outro pico próximo que pertence a um gama contaminante (caso de dubletos). Uim exemplo da descontaminação de um pico ajustado é mostrado na figura 3.16 . 


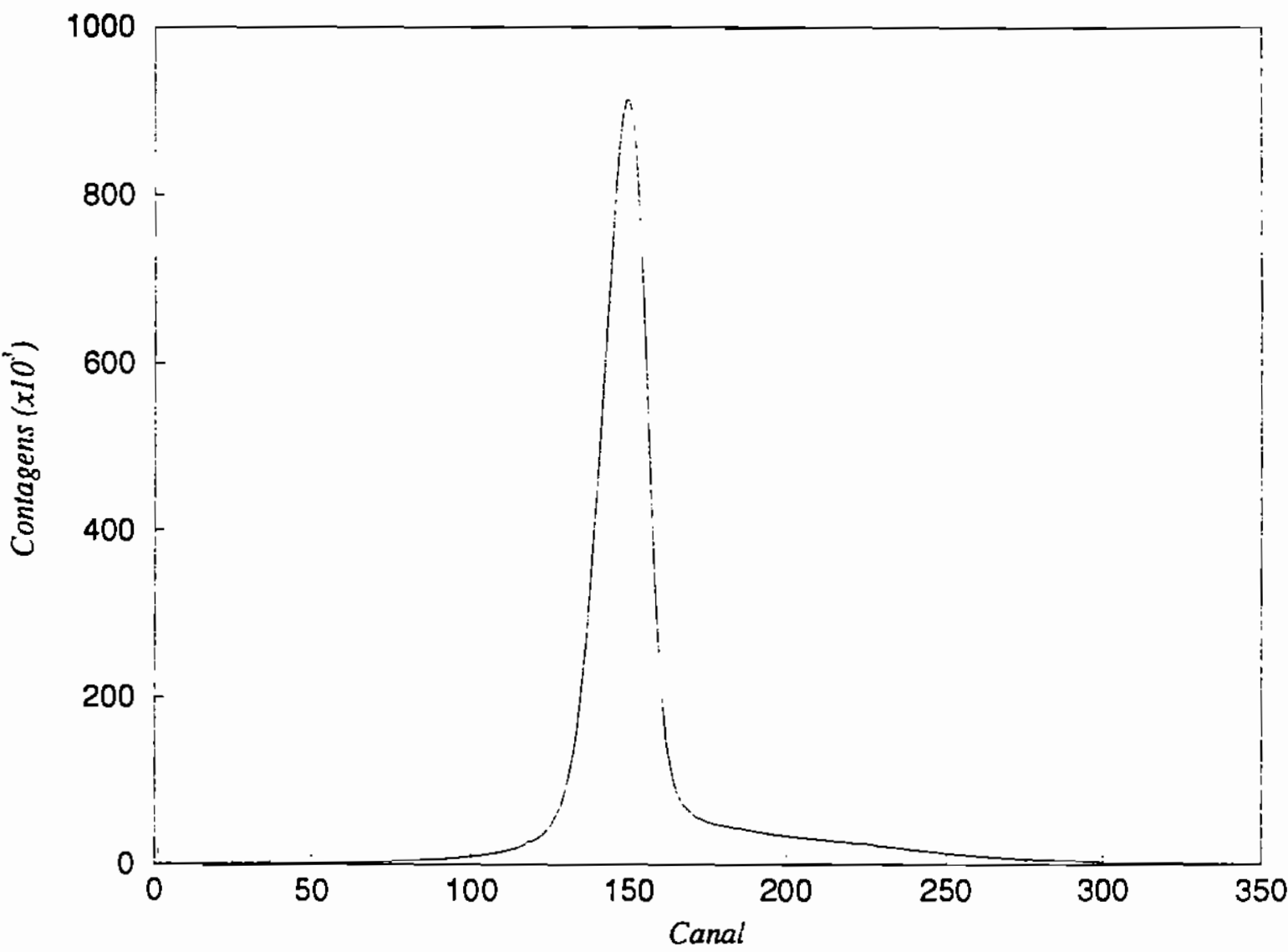

Figura 3.15: Espectro de projeção total da matriz $\gamma-t$ da medida de coincidéncia $\gamma-\gamma-t$ da reação ${ }^{128} \mathrm{Te}\left({ }^{14} \mathrm{~N}, 4 \mathrm{n} \gamma\right){ }^{138} \mathrm{Pr}$ na energia de $63 \mathrm{MeV}$.

A janela de energia $199 \mathrm{keV}$ mostra que este gama é comum aos três canais abertos na reação ${ }^{128} \mathrm{Te}+{ }^{14} \mathrm{~N}$. Existem coincidências fortes deste gama com as transiçōes de ${ }^{138} \mathrm{Pr}$, como também coincidências bem mais fracas com as de ${ }^{137} \mathrm{Pr}$ e ${ }^{139} \mathrm{Pr}$. Já o gama de $187 \mathrm{keV}$ pertence tanto ao núcleo de ${ }^{138} \mathrm{Pr}$ como de ${ }^{137} \mathrm{Pr}$.

Algumas janelas típicas obtidas com este código são apresentadas nas figuras 3.17 e 3.18. No núcleo de ${ }^{137} \mathrm{Pr}$, há duas transições de 230 e $331 \mathrm{keV}$, coincidentes entre si, que depopulam um estado isomérico de $2.7 \mu \mathrm{s}$. Devido a esta meia vida tão longa a contribuição do canal ${ }^{137}$ Pr na janela de $332 \mathrm{keV}$ foi praticamente eliminada pelo filtro de multiplicidade, como se pode observar na figura 3.18 a ausência do gama de $230 \mathrm{keV}$. 


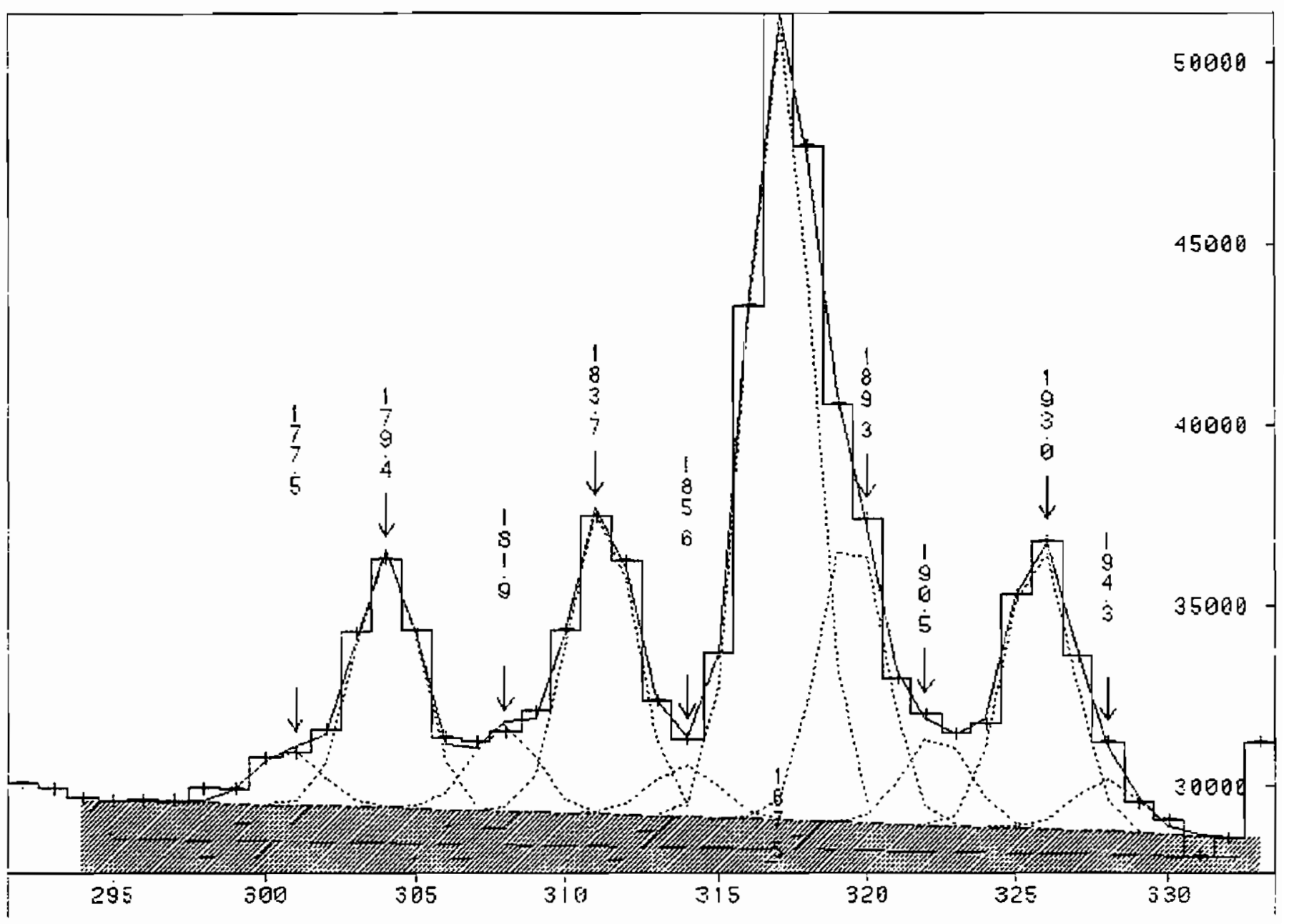

Figura 3.16: Exemplo de descontarninaçāo de um gama no espectro de projeção total. 
Após a construção de todas as janelas pertinentes ao núcleo de ${ }^{138} \mathrm{Pr}$ construiu-se uma tabela de áreas dos gamas coincidentes em cada janela. $O$ critério de aceitação das áreas (A) desta tabela, para a indicação da coincidência $\gamma-\gamma$, foi feito de acordo com o desvio padrão $(\sigma)$ do seguinte modo:

$$
\begin{aligned}
A>3 \sigma & \rightarrow \text { É quase certa a coincidência } \\
A<2 \sigma & \rightarrow \text { Não existe a coincidência } \\
2 \sigma<A & <3 \sigma \rightarrow \text { A questāo é indefinida. }
\end{aligned}
$$

As intensidades relativas de cada gama nas diferentes janelas foram obtidas através da razão entre a área (tabela de áreas de gamas em coincidência) em cada janela e da correspondente eficiência total. A eficiência total entre os detetores é dada pelo produto da eficiência do gama pela eficiência do "gate" de cada detetor, conforme a relação:

$$
\epsilon_{T}=\sum_{i \neq j} \epsilon_{i}(g) \cdot \epsilon_{j}(\gamma)
$$

onde $(\mathrm{g})$ se refere à janela e $(\gamma)$ à transição gama.

As intensidades dos gamas coincidentes no núcleo de ${ }^{138} \mathrm{Pr}$ corrigidas pela eficiência total dos 4 detetores utilizados, e normalizadas pelo gama de $199 \mathrm{keV}$, são apresentadas na tabela 3.1 e 3.2. Neste cálculo de intensidade relativa, levou-se em conta os coeficientes de conversāo interna (CCI), que afetam bastante os gamas de baixa energia, isto é, aproximadamente $50 \%$ para gamas da ordem de $100 \mathrm{keV}$ (M1) e $10 \%$ para gamas de $200 \mathrm{keV}$ (M1).

Com a análise dos espectros janelados de coincidência $\gamma-\gamma$ obtidos pelos código acima, bem como a análise da tabela de intensidades dos gamas foi possível a construção do esquema de níveis (apresentado no próximo capítulo). 

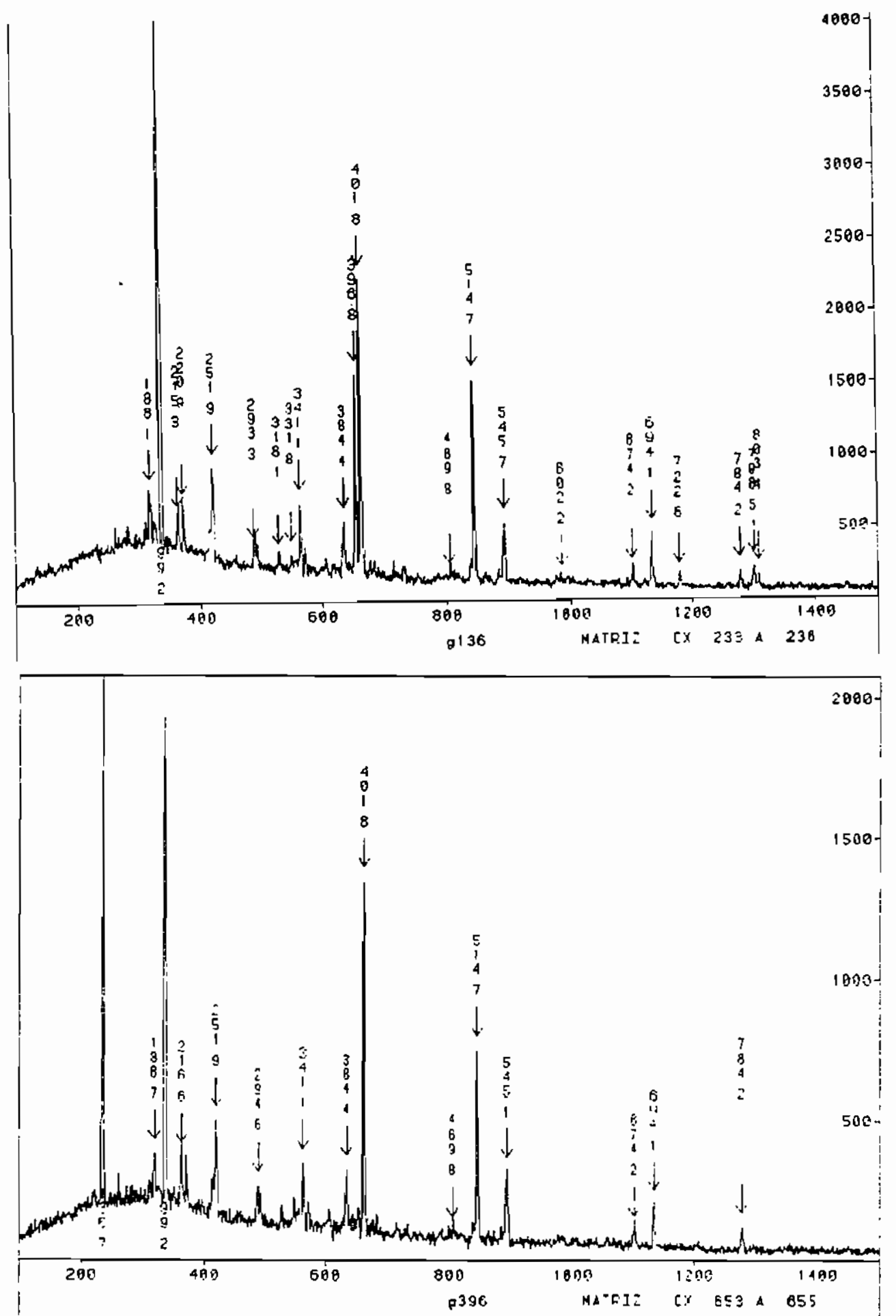

Figura 3.17: Espectros janelados da experiência de coincidência $\gamma-\gamma-t$ da reação $\left.{ }^{128} \mathrm{Te}\left({ }^{14} \mathrm{~N} .4 \mathrm{n} \gamma\right){ }^{138} \mathrm{Pr}, \mathrm{a}\right)$ janela na energia de $\left.136 \mathrm{keV} ; \mathrm{b}\right)$ janela na energia de $396 \mathrm{keV}$. 

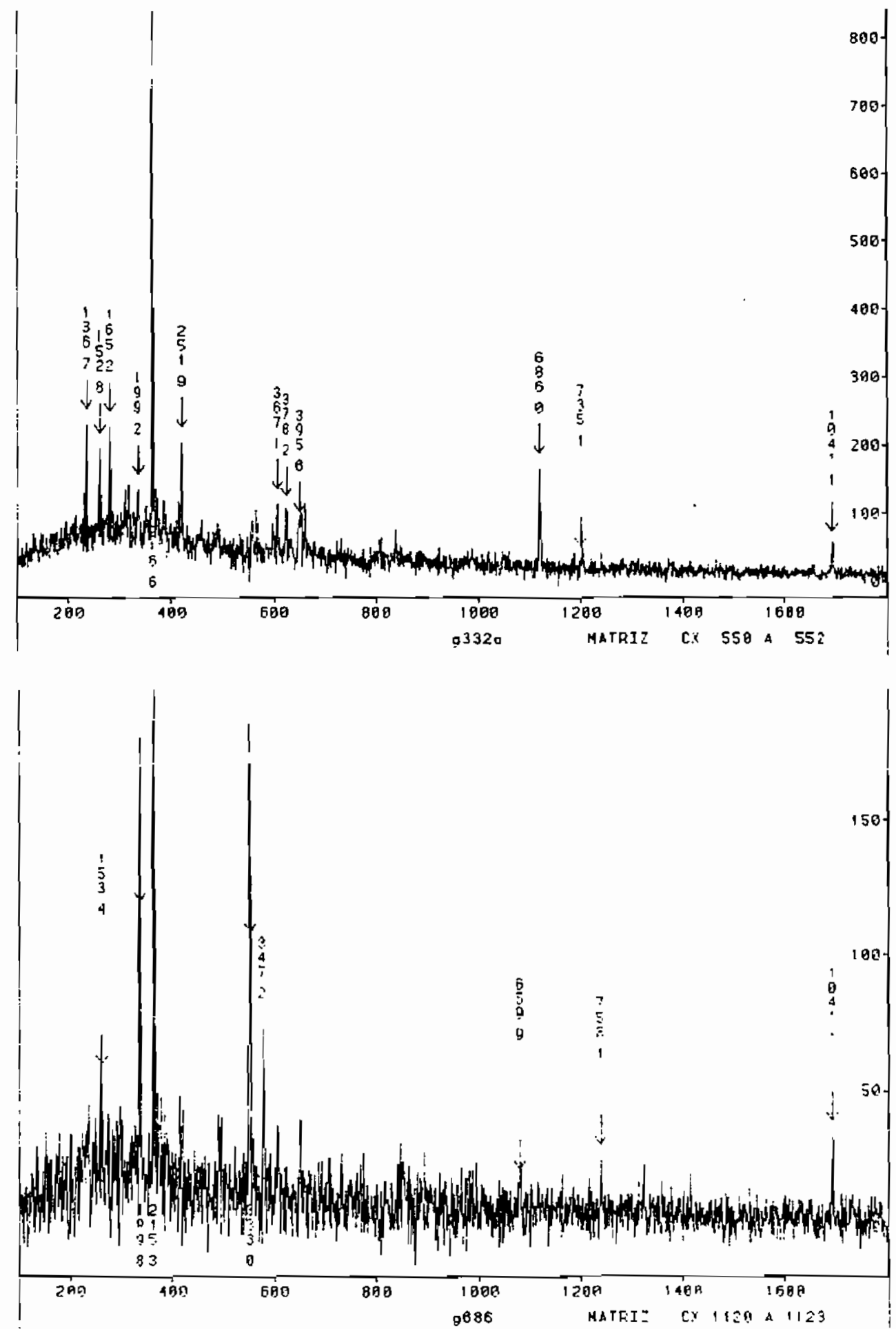

Figura 3.18: Espectros janelados da experiência de coincidência $\gamma-\gamma-t$ da reação ${ }^{128} \mathrm{Te}\left({ }^{14} \mathrm{~N}, 4 \mathrm{n} \gamma\right){ }^{136} \mathrm{Pr}_{\mathrm{r}}$, a)janela na energia de $332 \mathrm{keV}$; b) janela na energia de $686 \mathrm{keV}$. 
Tabela 3.1: Energias, Intensidades e Valores de $a_{2} / a_{0}$ e $a_{4} / a_{0}$ das distribuiçōes angulares para as transiçōes $\gamma$ da reação ${ }^{128} \mathrm{Te}\left({ }^{14} \mathrm{~N}, 4 \mathrm{n} \gamma\right){ }^{138} \mathrm{Pr}$.

\begin{tabular}{|c|c|c|c|c|c|}
\hline $\begin{array}{c}\text { Energia } \\
(\mathrm{keV})\end{array}$ & $\begin{array}{c}\text { Transiçāo } \\
J_{i} \rightarrow J_{f}\end{array}$ & $\begin{array}{r}\text { Intensidades } \\
\text { Relativas }\end{array}$ & $a_{2} / a_{0}$ & $a_{4} / a_{0}$ & Mult. Atrib. \\
\hline 136 & $9^{+} \rightarrow 8^{+}$ & $116.7(1.2)$ & $-0.23(1)$ & $-0.13(1)$ & M1 \\
\hline $153^{b}$ & - & $7.6(5)$ & - & - & - \\
\hline $166^{b}$ & $8^{+} \rightarrow 9^{-}$ & $5.3(5)$ & - & - & - \\
\hline $187^{a}$ & $12^{-} \rightarrow 11^{-}$ & $15.8(6)$ & - & - & - \\
\hline $189^{a}$ & - & $6.6(5)$ & - & - & - \\
\hline $199^{c}$ & - & 100.0 & $-0.37(1)$ & $0.06(1)$ & - \\
\hline 216 & - & $22.0(8)$ & $-0.54(4)$ & $0.19(4)$ & - \\
\hline 222 & $13^{-} \rightarrow 13^{-}$ & $16.9(7)$ & $-0.29(6)$ & $-0.18(8)$ & - \\
\hline 252 & $13^{-} \rightarrow 12^{-}$ & $35.0(8)$ & $-0.37(3)$ & $0.07(4)$ & M1 \\
\hline 294 & $14^{-} \rightarrow 13^{-}$ & $17.2(7)$ & $-0.28(6)$ & $-0.14(7)$ & - \\
\hline 297 & - & $7.3(5)$ & $-0.49(12)$ & $-0.24(14)$ & - \\
\hline 319 & - & $<5$ & $-0.19(9)$ & $-0.09(12)$ & - \\
\hline $332^{c}$ & - & $19.2(9)$ & $-0.25(6)$ & $0.12(3)$ & - \\
\hline 341 & $14^{-} \rightarrow 13^{-}$ & $35.4(1.0)$ & $-0.57(3)$ & $0.19(3)$ & - \\
\hline 346 & $13^{+} \rightarrow 12^{+}$ & $9.2(7)$ & $-0.63(13)$ & $0.05(18)$ & - \\
\hline 349 & - & $7.8(7)$ & $0.17(4)$ & $0.49(6)$ & - \\
\hline 367 & - & $15.5(7)$ & $-0.64(9)$ & $0.17(10)$ & - \\
\hline 375 & - & $5.5(7)$ & $-0.15(10)$ & $0.02(12)$ & - \\
\hline $384^{a}$ & $15^{-} \rightarrow 14^{-}$ & $29.8(9)$ & $-0.54(7)$ & $0.15(8)$ & M1 \\
\hline $386^{a}$ & - & $11.7(7)$ & $-0.39(18)$ & $-0.10(22)$ & - \\
\hline 390 & - & $7.6(7)$ & $-0.67(11)$ & $0.48(13)$ & - \\
\hline 396 & $11^{+} \rightarrow 10^{+}$ & $76.9(1.5)$ & $-0.57(2)$ & $0.17(2)$ & M1 \\
\hline 402 & $10^{+} \rightarrow 9^{+}$ & $102.1(1.8)$ & $-0.46(2)$ & $0.10(2)$ & M1 \\
\hline $411^{b}$ & $15^{-} \rightarrow 14^{-}$ & $11.4(9)$ & - & - & - \\
\hline $415^{b}$ & - & $9.2(7)$ & - & - & - \\
\hline $492^{b}$ & $13^{-} \rightarrow 12^{+}$ & $13.5(9)$ & - & - & - \\
\hline
\end{tabular}


Tabela 3.2: Continuaçāo

\begin{tabular}{|c|c|r|r|r|c|}
\hline $\begin{array}{c}\text { Energia } \\
(\mathrm{keV})\end{array}$ & $\begin{array}{c}\text { Transição } \\
J_{i} \rightarrow J_{f}\end{array}$ & $\begin{array}{r}\text { Intensidades } \\
\text { Relativas }\end{array}$ & $a_{2} / a_{0}$ & $a_{4} / a_{0}$ & Mult. Atrib. \\
\hline 515 & - & $97.5(1.9)$ & $-0.36(2)$ & $0.23(3)$ & - \\
$526^{b}$ & $16^{-} \rightarrow 15^{-}$ & $7.3(9)$ & - & - & - \\
540 & $16^{-} \rightarrow 15^{-}$ & $13.5(9)$ & $-0.99(15)$ & $0.43(16)$ & - \\
545 & $12^{+} \rightarrow 11^{+}$ & $36.4(1.3)$ & $-0.68(3)$ & $0.40(4)$ & M1 \\
$560^{b}$ & - & $<5$ & - & - & - \\
$596^{d}$ & $11^{-} \rightarrow 11^{+}$ & - & - & - & - \\
$640^{b}$ & $14^{+} \rightarrow 13^{+}$ & - & - & - & - \\
675 & - & $20.1(1.1)$ & $-0.69(7)$ & $0.27(9)$ & - \\
686 & $11^{-} \rightarrow 9^{-}$ & $19.9(1.1)$ & $0.19(5)$ & $0.19(7)$ & E2 \\
694 & - & $34.6(1.3)$ & $-0.48(2)$ & $0.32(3)$ & - \\
$759^{b}$ & - & $6.7(9)$ & - & - & - \\
$784^{a}$ & $12^{-} \rightarrow 11^{+}$ & $20.2(1.1)$ & - & - & - \\
$798^{b}$ & $11^{+} \rightarrow 9^{+}$ & $16.9(1.1)$ & - & - & - \\
804 & - & $11.7(1.1)$ & $-0.27(13)$ & $-0.16(17)$ & - \\
$993^{b}$ & $11^{-} \rightarrow 10^{+}$ & $4.6(9)$ & - & - & - \\
1041 & $13^{-} \rightarrow 11^{-}$ & $18.7(1.3)$ & $0.19(5)$ & $0.10(7)$ & $\mathrm{E} 2$ \\
\hline
\end{tabular}

$a=$ dubleto $\quad c=$ gama pertencente ao ${ }^{137,138} \mathrm{Pr}$

$b=$ baixa intensidade $\quad d=\gamma$ pertencente a Ge-n. 


\section{Capítulo 4}

\section{Resultados Experimentais e}

\section{Teóricos}

\subsection{Esquema de Niveis do ${ }^{138} \mathrm{Pr}$}

O esquema de níveis proposto neste trabalho é apresentado na figura 4.1 e incorpora quase todas as transições observadas nos espectros de coincidências. A disposição das transições $\gamma$ neste esquema de decaimento foi baseada nas relaçōes de coincidência $\gamma-\gamma$, nas intensidades relativas nas janelas e nas multipolaridades medidas.

O esquema de níveis é constituído basicamente de três bandas principais. A banda designada por 1 é a mais intensa e consiste de fortes transicões dipolares e um único "cross-over" fraco de $798 \mathrm{keV}$. Observa-se também um pequeno "staggering" característico desta regiāo de massa. A banda 2 foi subdividida em dois ramos $2 a$ e $2 b$, para facilitar a discussão posterior. Esta banda possui transiçōes dipolares e nenhum "cross-over". $\mathrm{Na}$ terceira banda as transições 686 e 1041 possuem natureza quadrupolar, enquanto que 216 e $332 \mathrm{keV}$ são dipolares. Para as demais transiçōes desta banda (759, 367 e $153 \mathrm{keV})$, no entanto, não foi possível determinar as multipolaridades devido à baixa estatística e contaminaçāo destes gamas.

Além destas seqüências principais foram observados 3 conjuntos de raios gama: 804, 


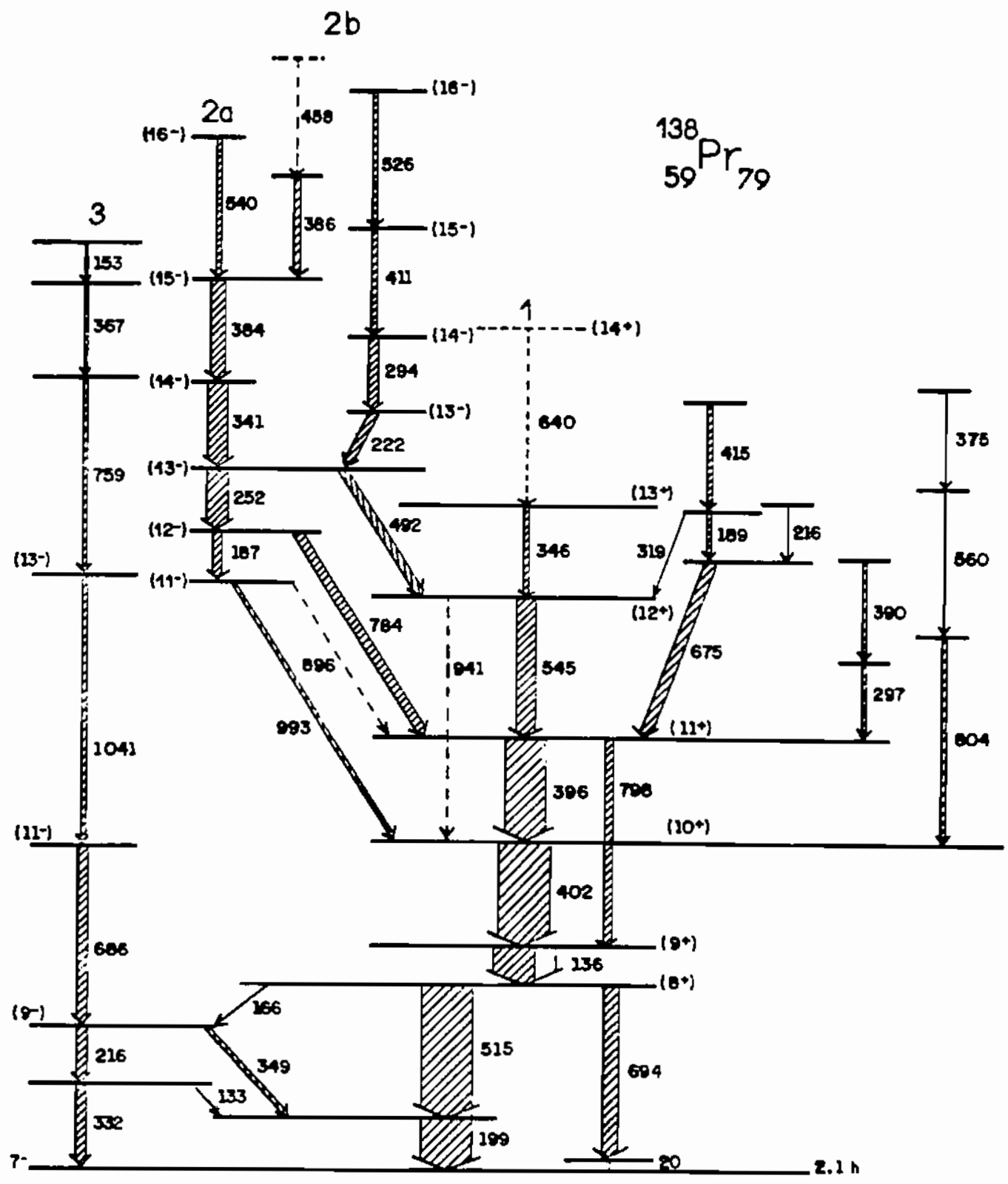

Figura 4.1: Esquema de níveis proposto para o núcleo de ${ }^{138} \mathrm{Pr}$, onde as energias das transiçōes são dadas em $\mathrm{keV}$. As atribuições de spins e paridades são discutidas na seção A.1.1 
560 e $375 \mathrm{keV} ; 297$ e $390 \mathrm{keV} ; 675,189,216$ e $415 \mathrm{keV}$ sendo que todos estes gamas fazem conexão com a banda 1 do ${ }^{138} \mathrm{Pr}$.

A análise cuidadosa dos espectros janelados de coincidência $\gamma-\gamma-t$ feitos sobre o espectro de projeção total possibilitou:

1) diferenciar os gamas distintos de 187 e $189 \mathrm{keV}$, que são dubletos na projeção total e não são coincidentes entre si. O gama de $187 \mathrm{keV}$ pertencente à banda 2a (segunda banda) é posicionado abaixo da transição $252 \mathrm{keV}$ e faz conexão com a banda principal (banda 1) através da transição de $993 \mathrm{keV}$, enquanto que o gama de $189 \mathrm{keV}$ mostra uma coincidência razoavelmente forte com 675,396 e os níveis abaixo deste.

2) Fazer a conexão entre a banda 2 (2a e 2b) com a banda principal (banda 1) através dos gamas de 492, 784, 993 e $596 \mathrm{keV}$ que sāo bastante fracos.

3) Concluir que há evidências de que acima do gama de $346 \mathrm{keV}$ existe uma transição de $640 \mathrm{keV}$ de baixíssima intensidade. Este gama foi observado somente nas janelas de $346 \mathrm{e}$ $136 \mathrm{keV}$.

4) Conseguir diferenciar os gamas de 384 e $386 \mathrm{keV}$ que estão em coincidência entre si e aparecem como dubleto na projeção total. Estes dois gamas fazem parte da banda 2 de ${ }^{138} \operatorname{Pr}$.

5) Observar que o gama de $694 \mathrm{keV}$ está em coincidência forte com a banda yrast (gamas de $136,402,396$ e $545 \mathrm{keV}$ ) e não está com os gamas de 515 e $199 \mathrm{keV}$. De acordo com o balanço de intensidade, é coerente supor que o gama de $694 \mathrm{keV}$ depopule o estado alimentado pelo gama de $136 \mathrm{keV}$, juntamente com o gama de $515 \mathrm{keV}$. Isto implica, ainda, na existência de um gama de $\approx 20 \mathrm{keV}$ em coincidência com $694 \mathrm{keV}$. No entanto, este gama de $20 \mathrm{keV}$ nāo foi observado por ser quase que completamente convertido devido à sua baixa energia, e por ser absorvido pelas paredes da câmara de espalhamento.

O gama de $187 \mathrm{keV}$ (banda 2) está em coincidência com $396 \mathrm{keV}$ (banda 1) e a conexāo entre estas bandas pode ser através do gama de $596 \mathrm{keV}$. No entanto, o gama de $596 \mathrm{keV}$ está situado, no espectro de projeção total, sobre uma estrutura proveniente do espalhamento de nêutrons nos ${ }^{74} \mathrm{Ge}$ (primeiro estado excitado de $596 \mathrm{keV}$ de $12 p s$ ) e ${ }^{76} \mathrm{Ge}$ (593 keV de 19ps) impossibilitando assim a construçāo de uma janela nesta energia. 
Para esclarecer dúvidas sobre a coincidência de $216 \mathrm{keV}$ com $675 \mathrm{keV}$ e não com $545 \mathrm{keV}$, e principalmente devido às relações de intensidades de população e depopulação de alguns níveis sugere-se o posicionamento duplo de $216 \mathrm{keV}$.

Existem ainda gamas como: 248, 271, 711, 723, 737, 764, 945 e $1014 \mathrm{keV}$ onde não foi possível posicioná-los consistentemente dentro do esquema de níveis. Como não foi observado o decaimento do estado populado pelo raio gama de 199 e $332 \mathrm{keV}$, é razoável supor que esse estado é o estado isomérico $I^{\pi}=7^{-}$de $T_{1 / 2}=2.1 h$, conhecido da literatura [34].

\subsubsection{Configurações e Spins das Bandas}

Através dos diagramas de Nilsson para prótons e nêutrons [27] apresentados na figura 4.2, nota-se que para os núcleos com $N<82$ e $Z>50$ a subcamada intrusa $h_{11 / 2}$ é a mais importante. O nível de Fermi, para esta região de massa situa o próton em torno de projeçōes pequenas $\left(\Omega_{p}=1 / 2\right)$ enquanto que o nêutron está em torno de $\Omega$ alto $\left(\Omega_{n}=11 / 2\right)$. Para o núcleo de ${ }^{138}$ Pr o valor de deformação $\beta \approx 0.14$ positivo foi calculado conforme descrito na seção 2.1.1. As possíveis configuraçōes de Nilsson próximas ao nível de Fermi, tanto para a deformação positiva como para a negativa, são apresentadas na tabela 4.1:

Tabela 4.1: Possíveis níveis de Nilsson para o núcleo de ${ }^{138} \operatorname{Pr}$ para as deformaçōes $\beta= \pm 0.14$

\begin{tabular}{|c|c|c|}
\hline$\beta$ & Prótons & Nêutrons \\
\hline$>0$ & {$[550] 1 / 2\left(h_{11 / 2}\right)$} & {$[505] 11 / 2\left(h_{11 / 2}\right)$} \\
& {$[413] 5 / 2\left(g_{7 / 2}\right)$} & {$[402] 3 / 2\left(d_{3 / 2}\right)$} \\
\hline$<0$ & {$[505] 11 / 2\left(h_{11 / 2}\right)$} & {$[501] 1 / 2\left(h_{11 / 2}\right)$} \\
& {$[411] 1 / 2\left(g_{7 / 2}\right)$} & \\
\hline
\end{tabular}

Analisando os esquemas de níveis dos núcleos ímpares da regiāo observa-se que os vi- 
zinhos ímpares em prótons, ${ }^{137} \operatorname{Pr}[8]$ e ${ }^{139} \operatorname{Pr}$ [36], mostram bandas yrast com transiçōes E2 "stretched" baseadas na configuração $\pi h_{11 / 2}$ desacoplado ( $\Omega$ baixo). Já para os núcleos ímpares em nêutrons, a situação é um pouco mais complicada e depende do número de nêutrons. Como o nêutron é fortemente acoplado, $\Omega=11 / 2$ ou $9 / 2$, é de se esperar que estes núcleos possuam transições M1 fortes e $\mathrm{E} 2$ fracas. De fato, os núcleos com $N \leq 77$, como por exemplo ${ }^{135} \mathrm{Ce}[37],{ }^{137} \mathrm{Nd}[38]$ e ${ }^{139} \mathrm{Sm}$ [39], apresentam bandas acopladas $(\Delta I=1)$ de paridade negativa construídas sobre o estado $11 / 2^{-}$com características fortemente perturbadas (figura 4.3a). No entanto, em $N=79$ como ${ }^{137} \mathrm{Ce}[10,11],{ }^{139} \mathrm{Nd}[12]$ e ${ }^{141} \mathrm{Sm}$ [13], foram observadas bandas desacopladas com seqūências $\Delta I=2$ de configuração $h_{11 / 2}$ e cabeça de banda 11/2- (figura 4.3b), típicas de deformaçōes negativas, isto é, forma oblata (vide diagrama de Nilsson para $\beta<0$ ). Estes dados experimentais acima mostram que $N=78$ é uma região de transição onde há uma mudança de forma de prolata $\left(\beta=0.22, \gamma>-30^{\circ}\right)$ para oblata $\left(\beta=0.15, \gamma<-30^{\circ}\right)$. Conseqüentemente pode-se esperar que no caso do núcleo duplamente ímpar de ${ }^{138} \mathrm{Pr}$ a banda yrast tenha configuração $\pi h_{11 / 2} \otimes \nu h_{11 / 2}$, podendo ser tanto prolata como oblata devido à simetria da subcamada intrusa $h_{11 / 2}$ (vide figura 4.2).

A análise dos núcleos ímpar-ímpar de $\mathrm{Pr}$ mostra que os isótopos ${ }^{130} \mathrm{Pr}[40],{ }^{132} \mathrm{Pr}$ [41], ${ }^{134} \operatorname{Pr}[42,43]$ e ${ }^{136} \operatorname{Pr}[3]$ apresentam banda yrast de configuração $\pi h_{11 / 2} \otimes \nu h_{11 / 2}$ caracterizadas por um pequeno "staggering", transiçōes M1 fortes e "cross-overs" E2 fracos típicas de bandas semi-desacopladas. A comparação da banda 1 do esquema de níveis do ${ }^{138} \mathrm{Pr}$ (figura 4.1) com bandas $\pi h_{11 / 2} \otimes \nu h_{11 / 2}$ destes isótopos (figura 4.4) mostra que esta se enquadra muito bem à sistemática da região. Pode-se ainda observar nesta figura que, quando o número de nêutrons aumenta, o espaçamento entre os níveis aumenta (diminuiçāo do "staggering") e isto revela uma diminuição do momento de inércia à medida que nos aproximamos da camada fechada $N=82$, indicando também um correspondente decréscimo da deformação $\beta$, como é de se esperar para esta região de massa. Deste modo, sugerimos a configuraçāo $\left(\pi h_{11 / 2} \otimes \nu h_{11 / 2}\right)$ para a banda 1 do ${ }^{138} \operatorname{Pr}$.

Devido ao posicionamento do nível de Fermi no diagrama de Nilsson $\left(\Omega_{n}\right.$ alto, $\Omega_{p}$ baixo $)$ o spin da cabeça de banda (banda 1) foi obtido através do acoplamento perpendicular entre 
a)

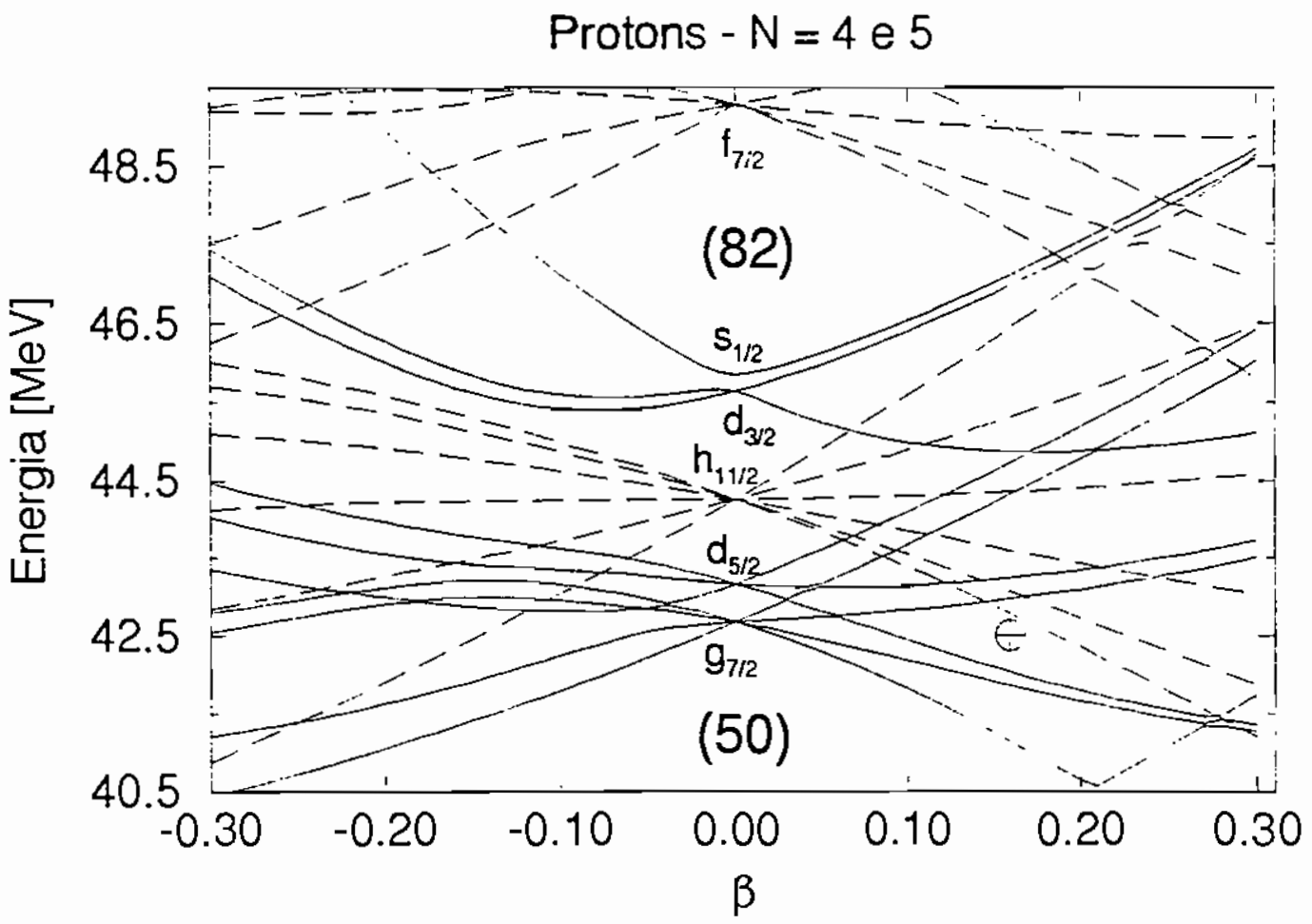

b)

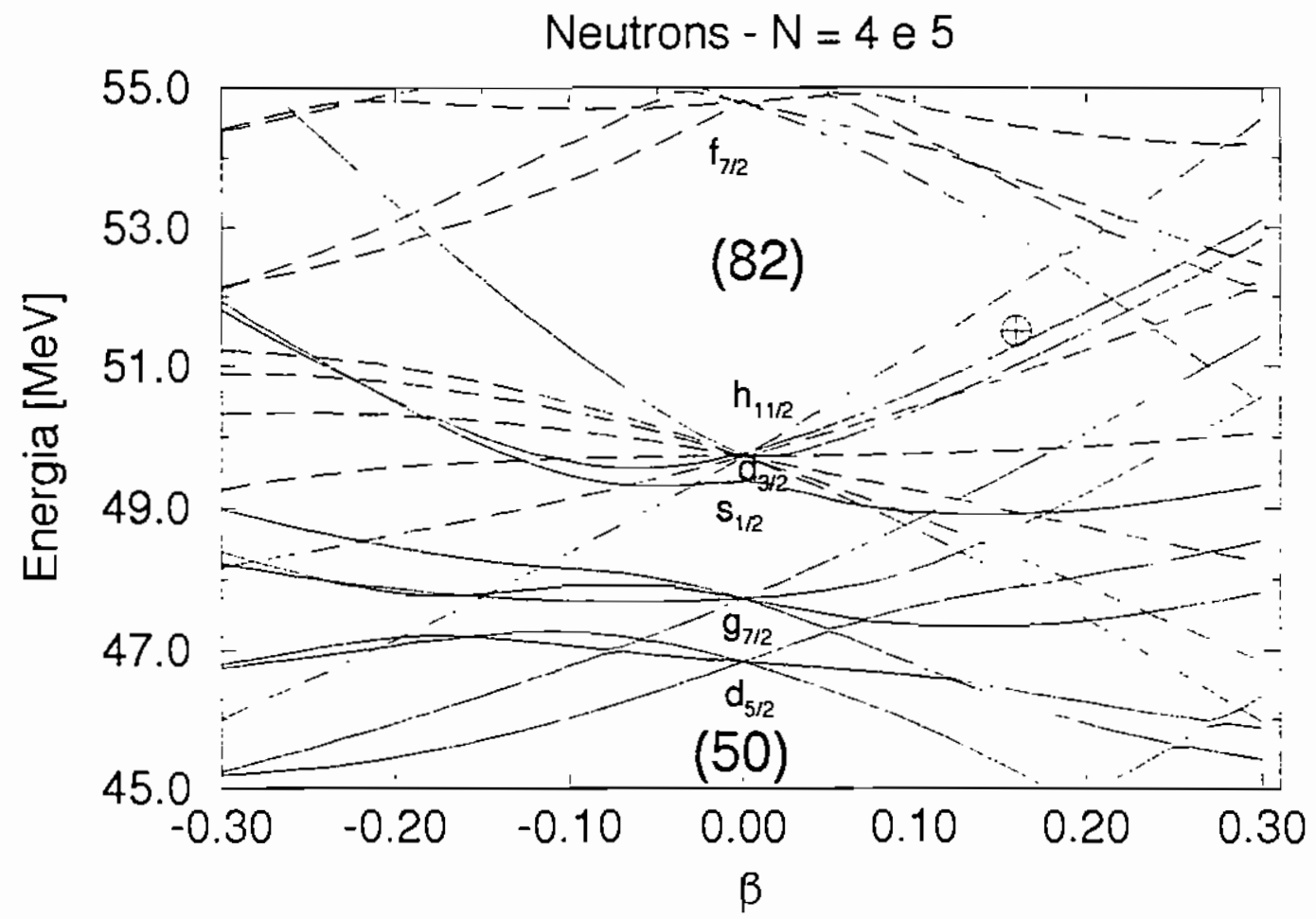

Figura 4.2: Níveis de Nilsson para as camadas $\mathrm{N}=4$ e 5 para a) prótons e b) nêutrons. 
a)
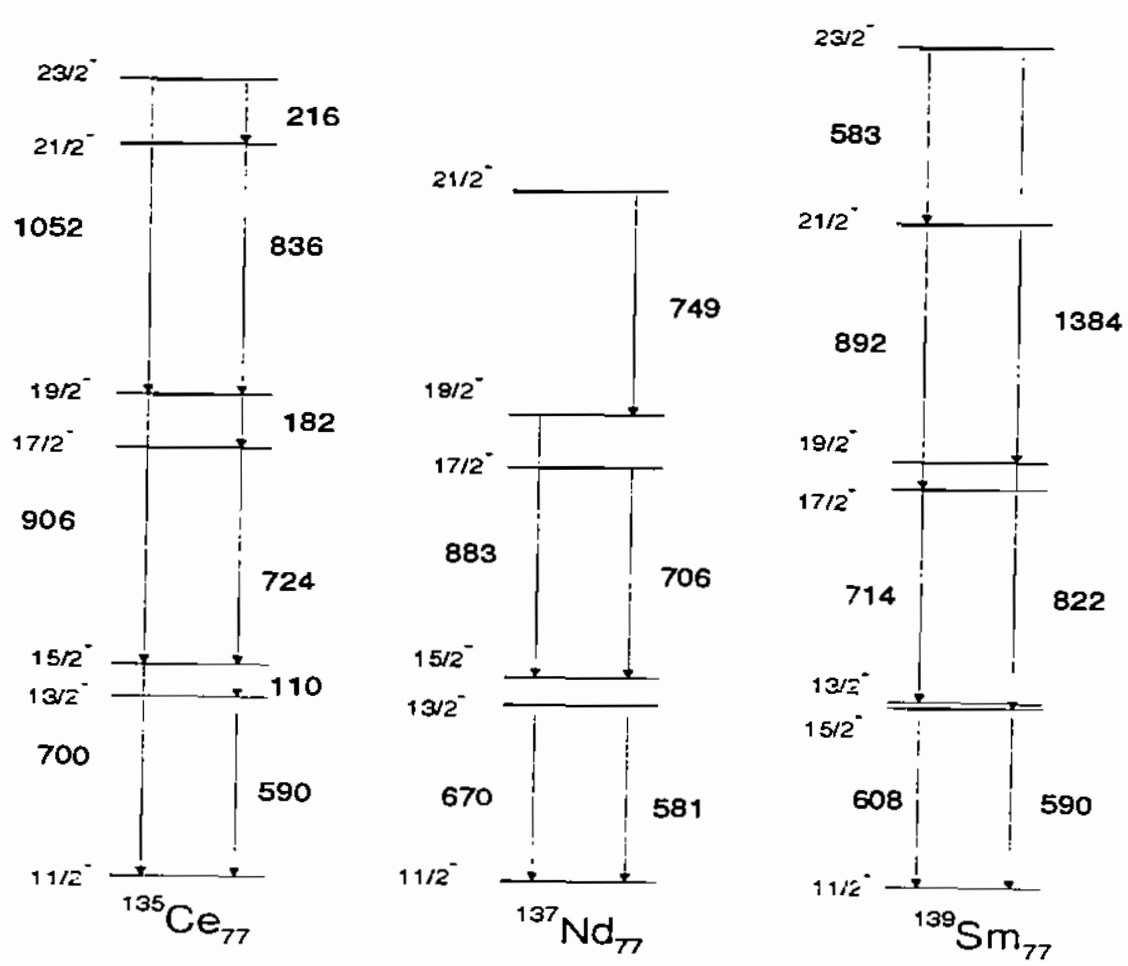

b)
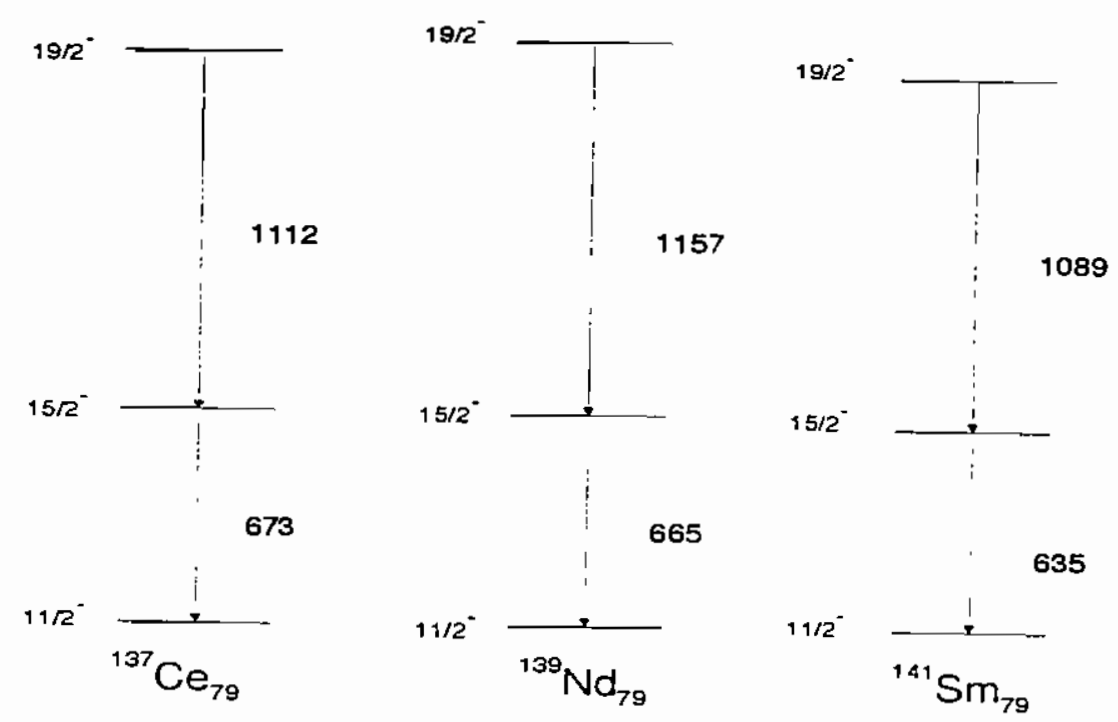

Figura 4.3: Sistemática de bandas $h_{11 / 2}$ acopladas e desacopladas dos isótonos ímpares em nêutrons a) $N=77:{ }^{135} \mathrm{Ce}[37],{ }^{137} \mathrm{Nd}[38],{ }^{139} \mathrm{Sm}[39]$ e b) $\mathrm{N}=79:{ }^{137} \mathrm{Ce}[10,11]$, ${ }^{139} \mathrm{Nd}[12],{ }^{1-41} \mathrm{Sm}[13$ :. 


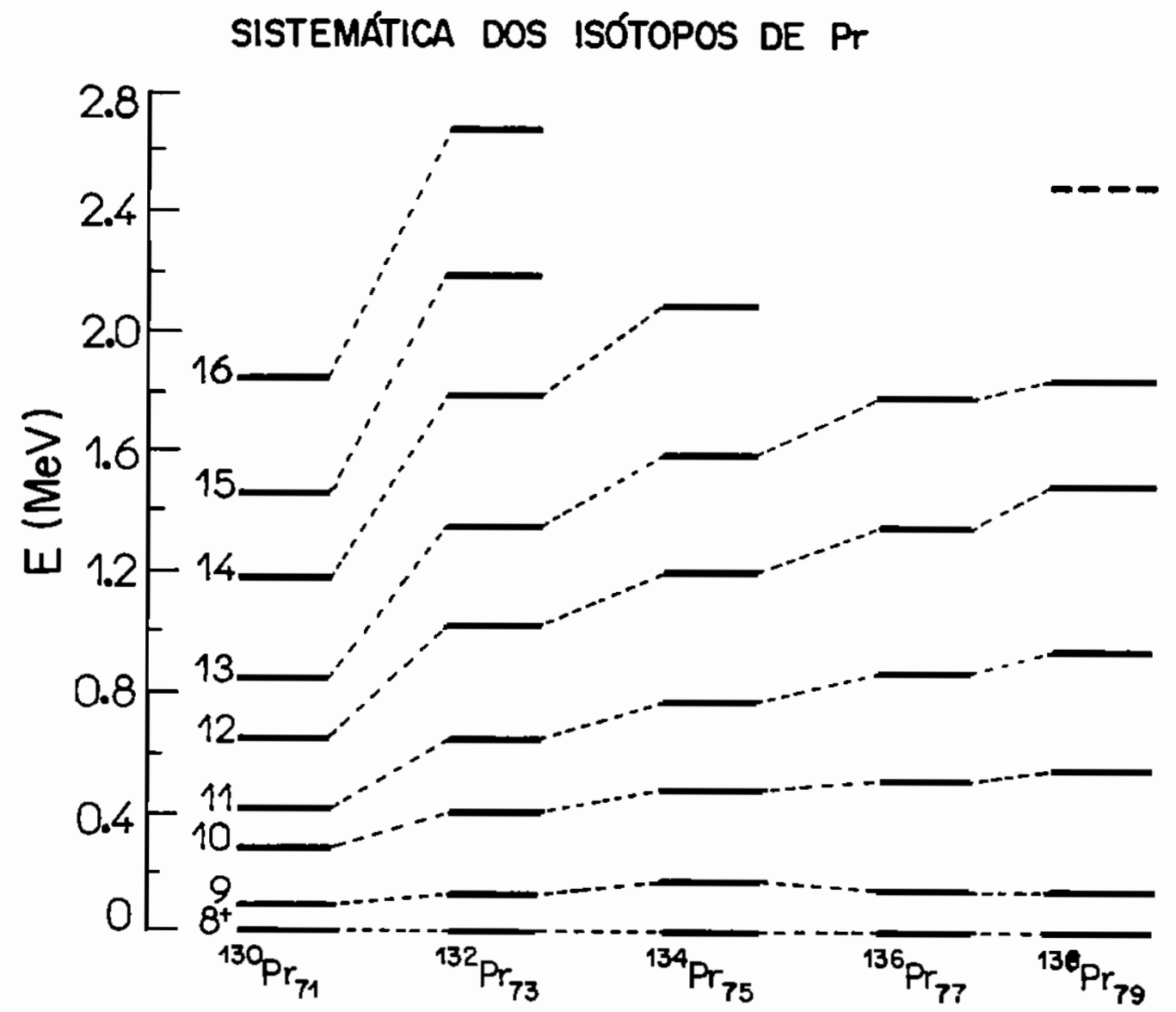

Figura 4.4: Sistemática de bandas $\pi h_{11 / 2} \otimes \nu h_{11 / 2}$ dos isótopos ímpar-ímpar de ${ }^{130} \mathrm{Pr}$ $[40],{ }^{132} \operatorname{Pr}[41],{ }^{134} \operatorname{Pr}[42,43],{ }^{136} \operatorname{Pr}[3]$ e ${ }^{138} \operatorname{Pr}$. As energias são normalizadas de tal modo que o nível $8^{+}$seja zero.

o nêutron e o próton, e é dado pela relação:

$$
I+1 / 2=\sqrt{\left(j_{p}+1 / 2\right)^{2}+\left(j_{n}+1 / 2\right)^{2}}
$$

resultando em $I=8^{+}$para $j_{p}=j_{n}=11 / 2$ (banda 1 ), de acordo também com a sistemática da região (figura 4.4).

Bandas similares à banda 2 a foram observadas nos núcleos vizinhos de ${ }^{134} \mathrm{Pr}_{\mathrm{r}}[42,43] \mathrm{e}$ seu isótono ${ }^{136} \mathrm{Pm}$ [42] além de um outro vizinho ímpar-ímpar ${ }^{138} \mathrm{Pm}$ [44] (figura 4.5). No caso do núcleo de ${ }^{136} \mathrm{Pm}$ esta banda tem configuraçāo $\pi[413] 5 / 2 \otimes \nu h_{11 / 2}$ que se cruza 
com uma banda de 4 quasi-partículas de configuração $\pi[413] 5 / 2 \otimes \nu h_{11 / 2} \otimes\left(\pi h_{11 / 2}\right)^{2}$, com um "backbend" na freqüência de $\hbar \omega \approx 0.28 \mathrm{MeV}$ (figura 4.6). No ${ }^{134} \mathrm{Pr}$, como no presente núcleo, ${ }^{138} \mathrm{Pr}$, foram observados somente os niveis acima do "backbend", e de acordo com a tabela 4.1, uma configuração possível para o núcleo de ${ }^{138} \operatorname{Pr}$ é $\pi[413] 5 / 2 \otimes \nu h_{11 / 2}$, o que concorda com os núcleos vizinhos dados acima. Portanto, estamos tentativamente atribuindo a mesma configuração de 4 quasi-partículas para a banda $2 a$ do ${ }^{138} \mathrm{Pr}$.

Atribuições de paridade e de spins para banda 2a foram baseadas na configuração $\pi[413] 5 / 2 \otimes \nu h_{11 / 2} \otimes\left(\pi h_{11 / 2}\right)^{2}$ e na alimentação desta banda para a banda yrast $\left(\pi h_{11 / 2} \otimes \nu h_{11 / 2}\right)$ do núcleo de ${ }^{138} \mathrm{Pr}$, onde assumiu-se que as transições de 492,784 e 993, que ligam a banda 2a com a banda yrast, sāo de natureza dipolar E1. Infelizmente, a multipolaridade dos gamas de 492 e $993 \mathrm{keV}$ nāo pode ser extraída dos dados de distribuição angular, pois ambos possuem baixa intensidade. Já o gama de $784 \mathrm{keV}$ faz parte de um tripleto (784, 787 e $788 \mathrm{keV}$ ) onde o gama em questão é o mais fraco deles, e a distribuição angular obtida não é confiável. No entanto, se o gama de $784 \mathrm{keV}$ fosse quadrupolar teria que ser M2, devido à mudança de paridade entre a banda 1 e 2 , com uma meia vida da ordem de $10^{-8}$ ou $10^{-9} \mathrm{seg}$, portanto um estado isomérico. $\mathrm{O}$ mesmo ocorreria para as transiçōes de 993 e $492 \mathrm{keV}$, onde a última deveria possuir meia vida de $\approx 10^{-7} \mathrm{seg}$. Isto levaria a um conjunto de três estados isoméricos consecutivos, porém isto é pouco provável. Sendo assim, acreditamos que as transições evidenciadas acima são dipolares, $\mathrm{E} 1$, com meia vida de $\approx 10^{-15} \mathrm{seg}$.

A estrutura chamada de $2 b$ não é muito clara e nos núcleos vizinhos nenhuma estrutura similar foi observada; no entanto, uma possível interpretação será discutida na seção 4.2.

Uma comparação da banda 3 de ${ }^{138} \mathrm{Pr}$ com os vizinhos ímpares em nêutrons ${ }^{137} \mathrm{Ce}$ $[10,11],{ }^{139} \mathrm{Nd}[12]$ e ${ }^{141} \mathrm{Sm}[13]$ que possuem bandas com transiçōes E2 "stretched" forte de configuração $\nu h_{11 / 2}$ e cabeça de banda 11/2- é mostrada na figura 4.7a. As energias das transições destas bandas são quase que idênticas às energias de 686 e $1041 \mathrm{keV}$ da banda 3 de ${ }^{138} \mathrm{Pr}$. Também na figura $4.7 \mathrm{~b}$ comparamos esta mesma banda com os vizinhos ímpares em prótons, isto é, ${ }^{139} \mathrm{Pr}$ e ${ }^{141} \mathrm{Pm}[36]$, onde se observa novamente gamas E2 "stretched" fortes e os autores sugerem uma estrutura oblata ou triaxial, indicando que o próton 


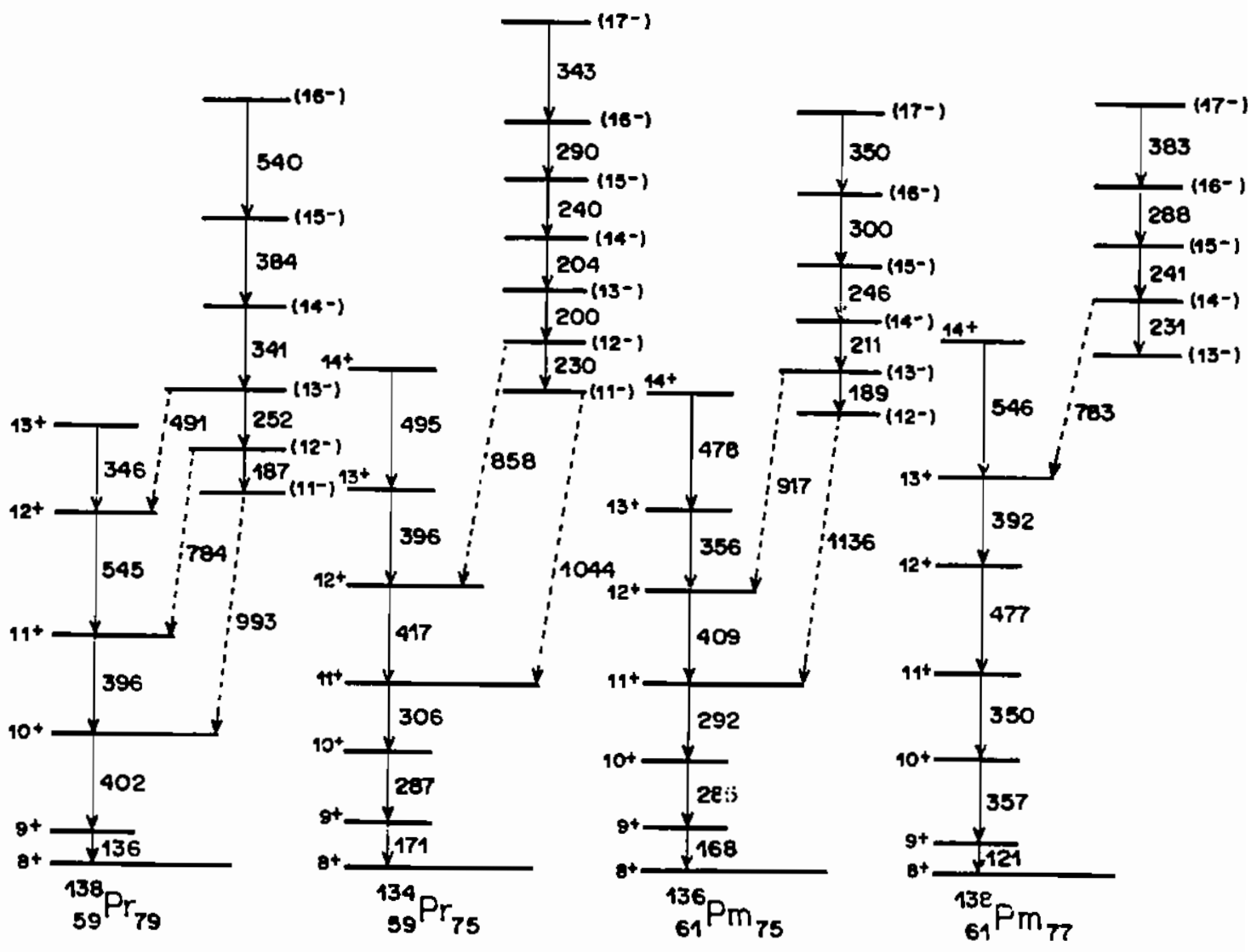

Figura 4.5: Bandas $\pi[413] 5 / 2 \otimes \nu h_{11 / 2} \otimes\left(\pi h_{11 / 2}\right)^{2}$ de ${ }^{134} \operatorname{Pr}[42,43],{ }^{136} \mathrm{Pm}[42] \mathrm{e}{ }^{138} \mathrm{Pm}$ [44] em comparação com a banda $2 a$ de ${ }^{138} \mathrm{Pr}$. 


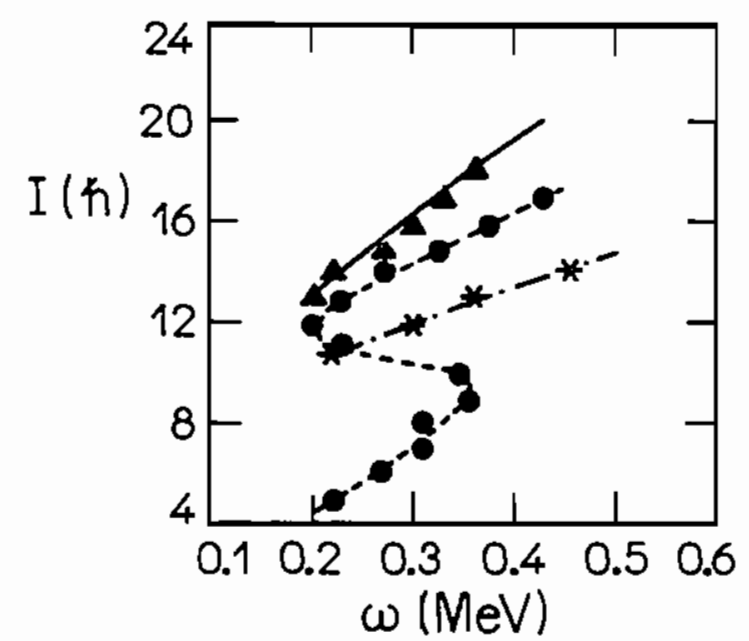

Figura 4.6: Curvas de $\omega \times \mathrm{I}$, para a banda $\pi[413] 5 / 2 \otimes \nu h_{11 / 2}$ dos núcleos de ${ }^{138} \operatorname{Pr}_{\mathrm{r}}$ (asterisco), ${ }^{136} \mathrm{Pm}$ (círculo) e ${ }^{134} \mathrm{Pr}$ (triângulo). "Backbending"ocorre $\hbar \omega \approx 0.28 \mathrm{MeV}$.

é desacoplado. O orbital mais próximo do nível de Fermi de acordo com a tabela 4.1 é [411]1/2 $1{ }^{\dagger}$ na deformação oblata. Deste modo, sugere-se a configuraçāo $\pi[411] 1 / 2 \otimes \nu h_{11 / 2}$ oblata para a banda 3 de ${ }^{138} \mathrm{Pr}$, com cabeça de bandia dada por:

$$
J^{\pi}=j_{p}+j_{n}=11 / 2+7 / 2=9^{-}
$$

\subsection{Cálculos de CSM}

Seguindo o procedimento descrito na seção 2.1.1 para transformar os dados experimentais do sistema laboratório para o sistema intrinseco do núcleo, inicialmente calcula-se o valor da projeçāo do momento angular $\langle K\rangle$ sobre o eixo de simetria, estimado segundo a regra de Gallagher-Moszkowski [19;. No caso da banda 1 de configuração $\pi[550] 1 / 2 \otimes \nu: 505] 11 / 2$ temos $\langle K\rangle=6$, e para a banda 2 com $\pi[413 j 5 / 2 \otimes \nu[505] 11 / 2$ temos $\langle K\rangle=3$ ou 8 .

A comparaçāo das razōes das probabilidades reāuzidas de transição B(M1)/B(E2) dos 
a)
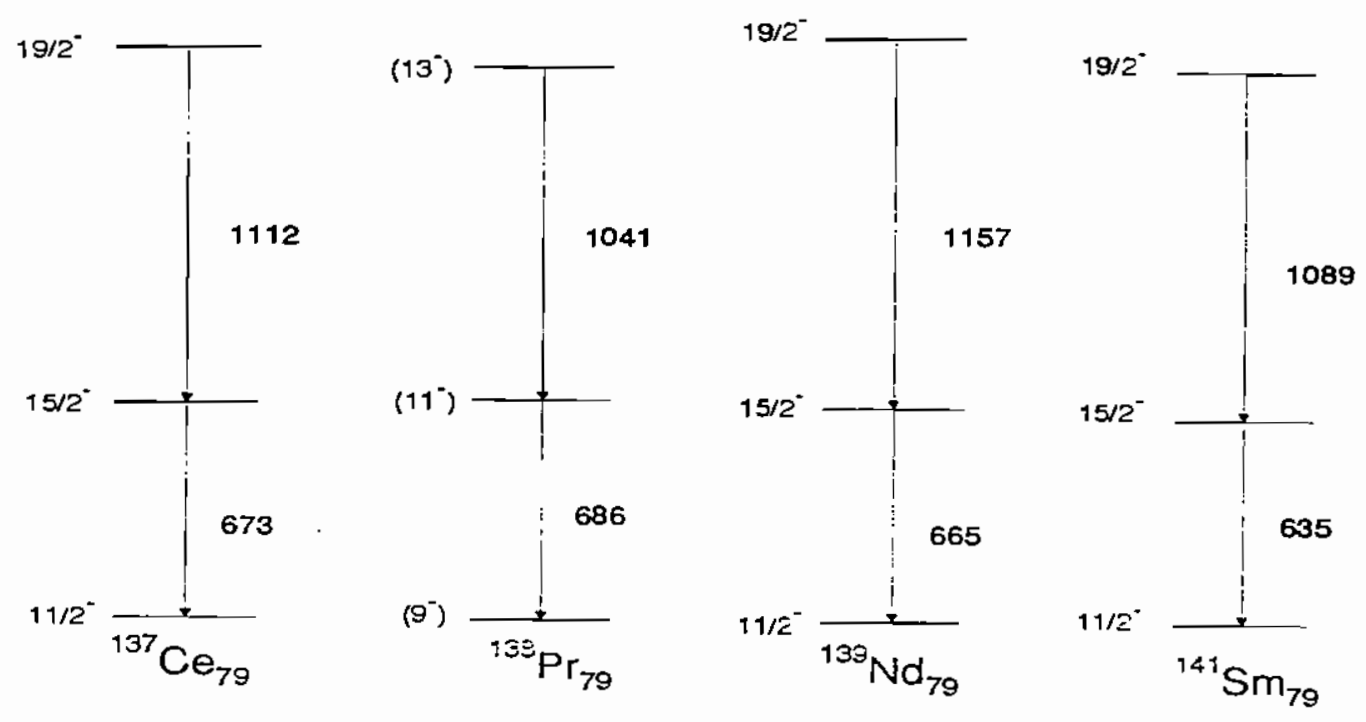

b)

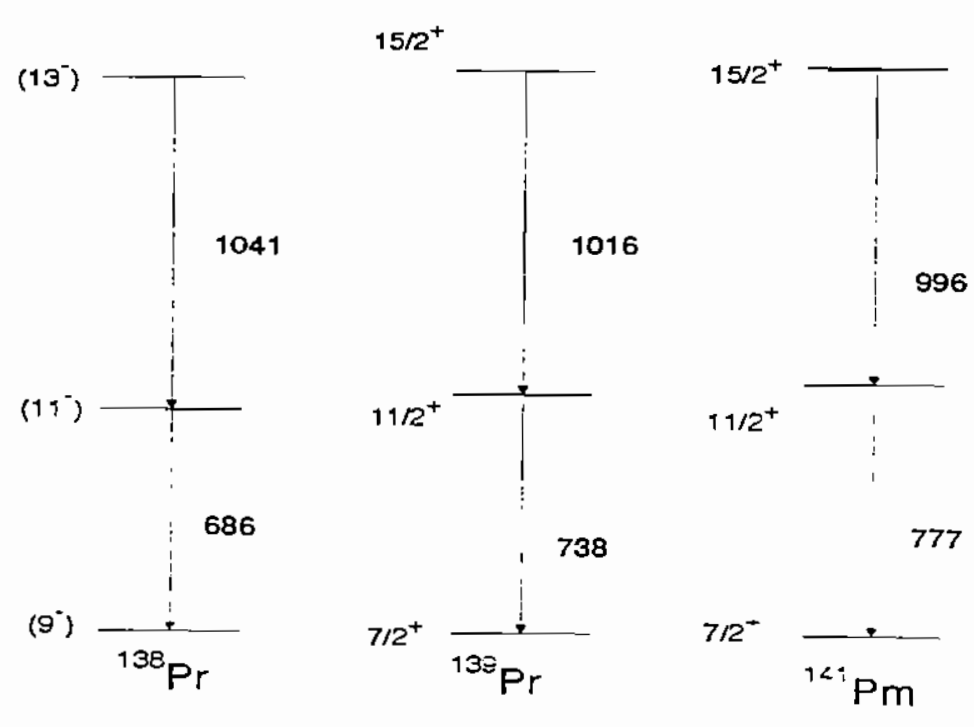

Figura 4.7: Esquema de niveis parciais a) dos isótonos vizinhos ímpares em nêutron ${ }^{137} \mathrm{Ce}$ $[10,11]$ e ${ }^{139} \mathrm{Nd}[12]$ e e ${ }^{141} \mathrm{Sm}[13]$; e b) dos vizinhos em prótons ${ }^{139} \mathrm{Pr}$ e ${ }^{141} \mathrm{Pm}[36]$, em comparação com a banda 3 do ${ }^{138} \mathrm{Pr}$. 
valores experimentais, com os valores teóricos do formalismo de Dönau e Frauendorf [45] permite estimar o valor da projeção $K$ de uma banda. No caso da banda 2, porém, nenhuma transição E2 foi observada e supondo-se que o limite superior para as intensidades destas transiçōes de interesse seja de $\approx 3$, obtém-se a razão $\mathrm{B}(\mathrm{M} 1) / \mathrm{B}(\mathrm{E} 2) \geq 15\left(\mu_{N} / e b\right)^{2}$ e $\geq 6\left(\mu_{N} / e b\right)^{2}$ para as bandas $2 \mathrm{a}$ e $2 \mathrm{~b}$ respectivamente. Os cálculos teóricos para a configuração da banda 2 usando o formalismo acima, mostraram que os valores das razōes são de $\approx 20\left(\mu_{N} / e b\right)^{2}$ para $K=3$ e $\approx 50\left(\mu_{N} / e b\right)^{2}$ para $K=8$, consistentes com os limites experimentais. Pela regra de Gallagher-Moszkowski atribuíu-se o valor de $K=3$ para a banda $2 \mathrm{a}$ e $K=8$ para a banda $2 b$.

Nos cálculos dos routhianos e alinhamentos experimentais os parâmetros de Harris foram extraídos ajustando-se a curva $I_{x}(\omega)=i_{x}(\omega)+\omega \mathcal{J}_{0}+\omega^{3} \mathcal{J}_{1}$ aos dados experimentais da banda yrast, isto é, aos valores abaixo do "backbend" onde $i_{x}$ é constante. Neste ajuste não se considerou a última transição $(640 \mathrm{keV})$ por ser duvidosa. Os valores encontrados, $\mathcal{J}_{0}=4.72 \hbar / \mathrm{MeV}, \mathcal{J}_{1}=28.87 \hbar^{4} / \mathrm{MeV}^{3}$ foram utilizados nos cálculos para todas as bandas deste núcleo. $O$ gráfico de $i_{x} \times \omega$ da figura 4.8a mostra que o alinhamento é aproximadamente constante, $i_{x}=6.07 \hbar$ para a banda 1 , e que para a banda 2 este valor está em torno de $11 \hbar$, no intervalo de freqüência observada (máxima de $0.45 \mathrm{MeV}$ ). Os valores dos routhianos experimentais sāo mostrados no gráfico de $e^{\prime} \mathrm{x} \omega$ para ambas as bandas (figura 4.8b), onde se observa que para a banda yrast (banda 1) as curvas com "signatures" $\alpha=-1$ e $\alpha=0$ possuem um "signature splitting" de $\Delta e^{\prime} \approx 50 \mathrm{keV}$ na freqüência de $\hbar \omega \approx 350 \mathrm{keV}$, tendendo a zero para valores menores, enquanto que para a banda 2 este valor está em torno de $\Delta e^{\prime} \approx 25 \mathrm{keV}$ mantendo -se constante.

Foram realizados também cálculos de routhianos teóricos de quasi-partícula única em camada de alto $j$, em função da rotação $\omega$ e da deformação $\gamma$. Para estes cálculos utilizouse um código denominado "Cranking", onde os parâmetros utilizados, como deformaçāo $\beta$, parâmetros de Nilsson, nível de Fermi, etc., foram estabelecidos conforme seção a 2.1 . Os resultados típicos dos routhianos de quasi-partículas em funçāo de $\omega$, para $\gamma=0$, do núcleo de ${ }^{138} \mathrm{Pr}$ estāo apresentados na figura 4.9. Nota-se através desta figura que o primeiro cruzamento para prótons (figura 4.9a) está em torno de $0.038 \hbar \omega_{0}=0.30 \mathrm{MeV}$, 
a)

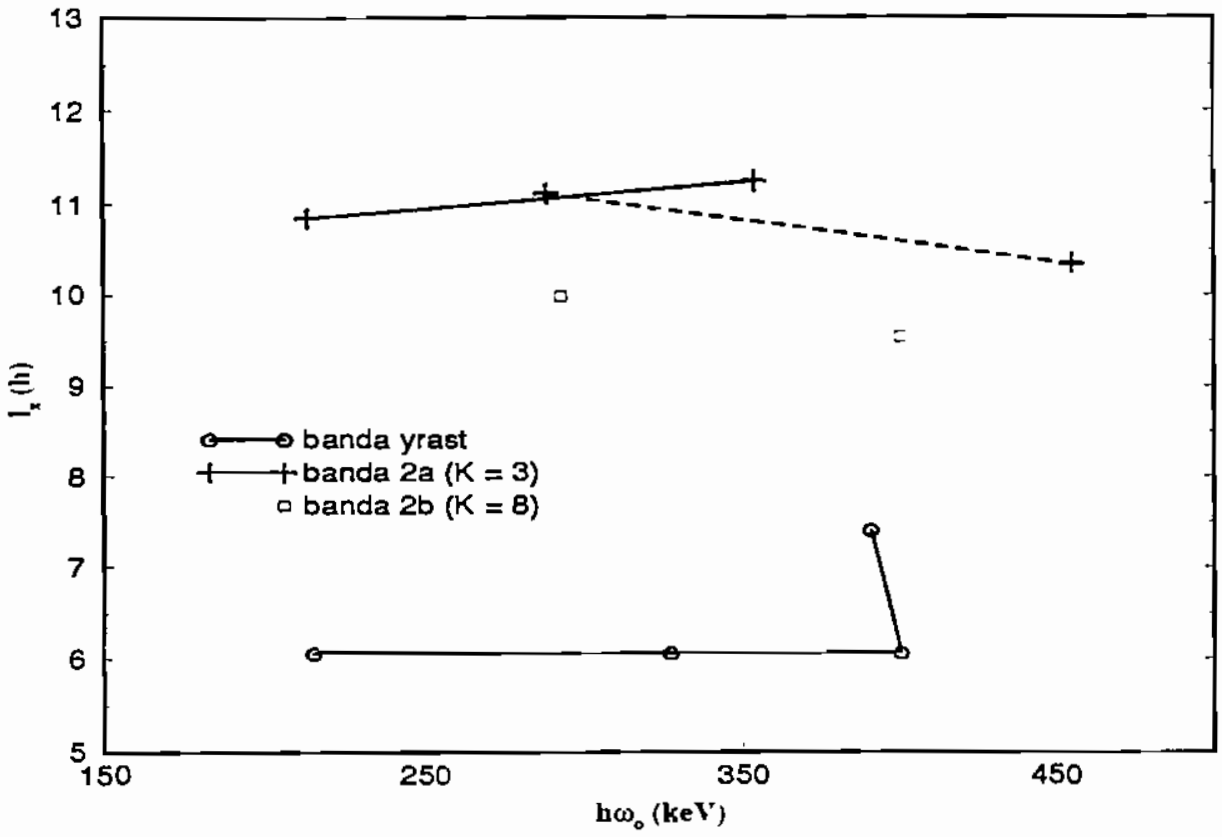

b)

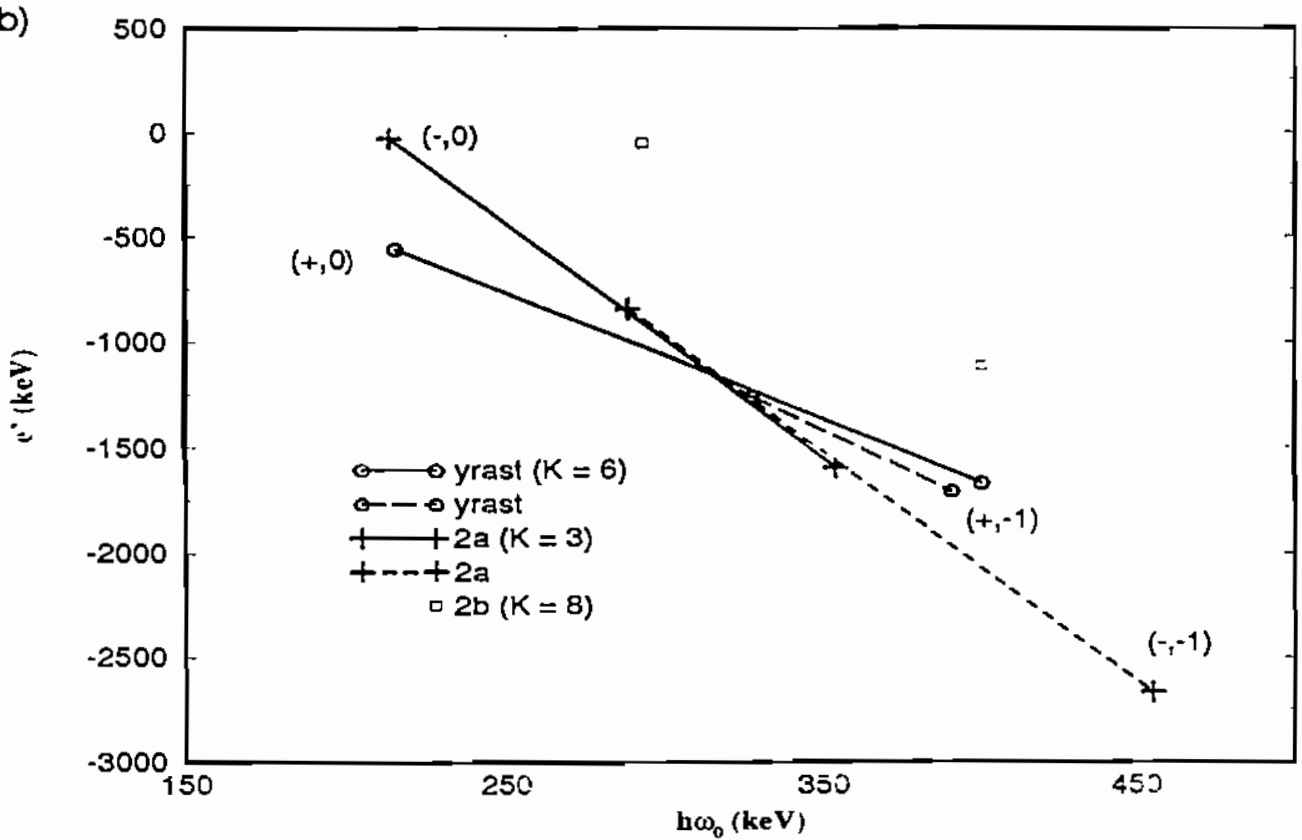

Figura 4.8: (a) aỉnhamentos e (b) routhianos experimentais em função da frequència de rotação, $\hbar \omega_{0}$, para a banda 1 e $2 \mathrm{a}$ de configuraçōes $\pi h_{11 / 2} \otimes \nu h_{11 / 2}(\mathrm{~K}=6)$ e $\pi\left[413_{\mathrm{j}} 5 / 2 \otimes \nu h_{11 / 2} \otimes\left(\pi h_{\mathrm{J} 1 / 2}\right)^{2}(\mathrm{~K}=3)\right.$, respectivamente, do núcleo de ${ }^{138} \mathrm{Pr}$. 
enquanto que o de nêutron (figura 4.9b) está em torno de $0.055 \hbar \omega_{0}=0.44 \mathrm{MeV}$ (ambos para a camada $h_{11 / 2}$ ). Este cruzamento para o próton corresponde ao spin $\approx 12^{+}$da banda yrast, e após este cruzamento o nível [413]5/2 se torna o mais provável, figura 4.9a. Assim atribuiu-se a configuração $\pi[413] 5 / 2 \otimes \nu h_{11 / 2} \otimes\left(\pi h_{11 / 2}\right)^{2}$ à banda 2 que se encontra um pouco acima do spin $12^{+}$e corresponde a excitaçōes de 4 quasi-partículas.

Nesta figura, a separação entre as "signatures" do próton no orbital $h_{11 / 2}$ é de $\approx 600 \mathrm{keV}$; sendo assim, é de se esperar que a banda yrast seja determinada apenas pelo acoplamento da "signature" favorecida do próton com ambas as "signatures" do nêutron, enquanto que a banda formada pela "signature" desfavorecida do próton nāo deverá ser observada experimentalmente por situar-se em energia muito elevada. Já os cálculos dos routhianos para a configuração $\pi$ [413]5/2 mostram que a separação entre as duas "signatures" neste caso, é menor que $100 \mathrm{keV}$, logo é de se esperar que experimentalmente possam ser observadas ambas as "signatures" do próton combinadas com ambas do nêutron, sugerindo duas bandas próximas. As bandas 2a e $2 \mathrm{~b}$ medidas experimentalmente parecem se enquadrar nesta descrição.

Os cálculos dos routhianos de partícula única, $e_{p}^{\prime}$ e $e_{n}^{\prime}$, em função da deformação $\gamma$ para $\hbar \omega=0.25 \mathrm{MeV}$ dos estados $h_{11 / 2}$ de energia mais baixa do ${ }^{138} \mathrm{Pr}$, figura 4.10 , mostram as tendências polarizadoras do próton e do nêutron típicas desta regiāo. $O$ próton ( $A$ e $B$ ), tende para valores de $\gamma>0^{\circ}$ enquanto o nêutron (a e b), para $\gamma \approx-60^{\circ}$. Já o próton do orbital $g_{7 / 2}$ (E e F) tende também para um mínimo muito mais raso $\left(\gamma=-60^{\circ}\right)$. A banda 3 observada experimentalmente pode então ser devido à combinação do nêutron $h_{11 / 2}$ com o próton neste novo orbital, [411]1/2, o que resultaria em uma banda oblata com um mínimo em torno de $\gamma \approx-60^{\circ}$.

O routhiano total ( $E_{\text {tol }}^{\prime}$ ) que considera a contribuição do caroço e os routhianos das quasi-partículas (próton e nêutron), foi calculado seguindo o procedimento da seção 2.1. Os resultados destes cálculos em função de $\gamma$, para a freqüência de $\omega=0.03 \hbar \omega_{0} \approx 0.25 \mathrm{MeV}$ e para os primeiros estados de $h_{11 / 2}$ (figura 4.11) mostram um mínimo em torno de $\gamma_{2}=10^{\circ}$ prolato para a banda $\pi h_{11 / 2} \otimes \nu h_{11 / 2}$, com o valor de "signature splitting" nulo, de acordo com o resultado experimental. 

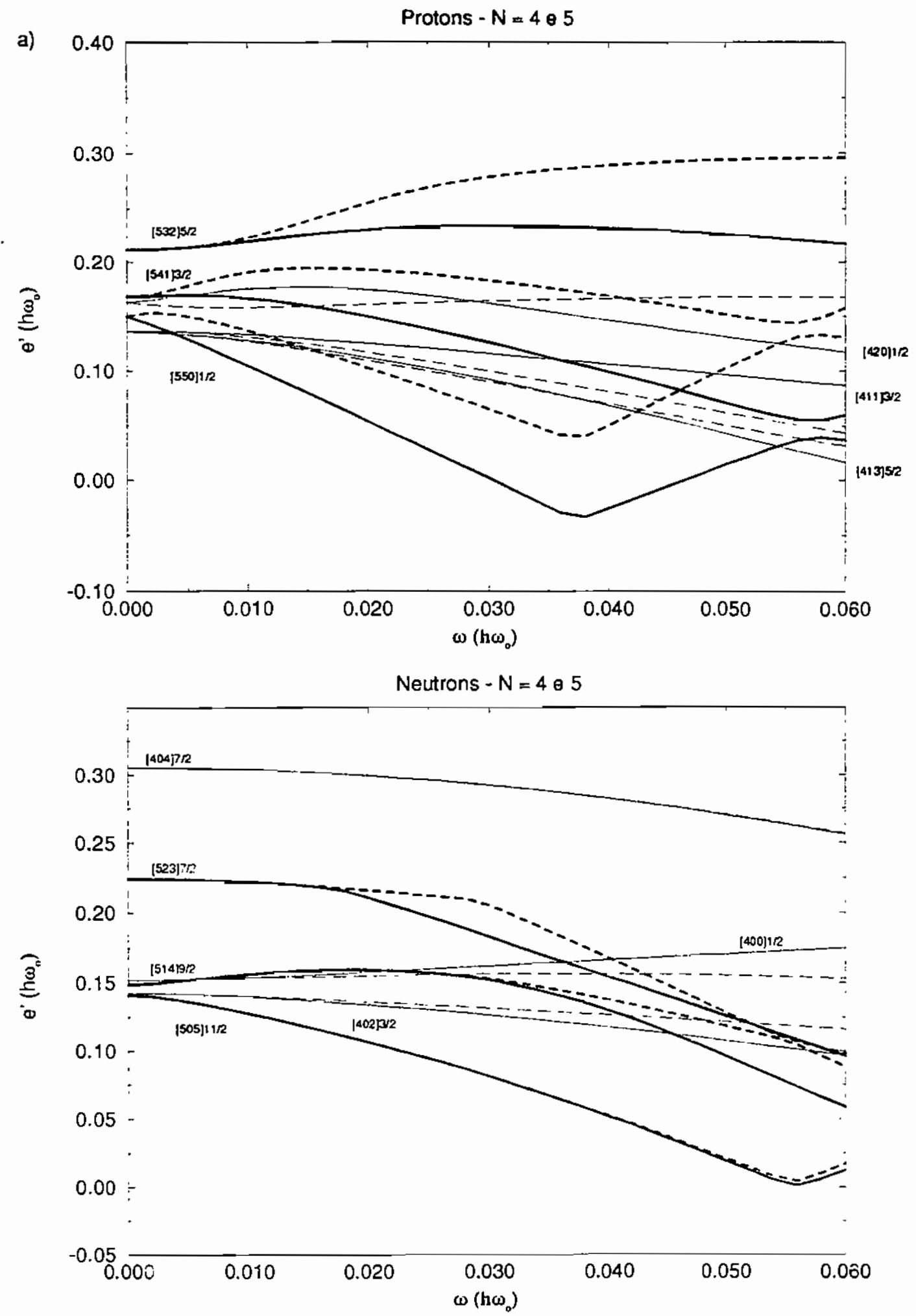

Figura 4.9: Níveis de energia de quasi-partículas calculados, e', em função da frequência de rotaçāo, $\hbar \omega$, (a) prótons e (b) nêutrons. Os parâmetros utilizados foram $\beta=0.14$, $\Delta \approx 1.0 \mathrm{MeV}, \lambda_{p}=5.62 \hbar \omega_{\mathrm{i},}, \lambda_{n}=6.10 \hbar \omega_{0}, \gamma=0^{\circ},\langle Z\rangle=59 \mathrm{e}\langle N\rangle=79$. 


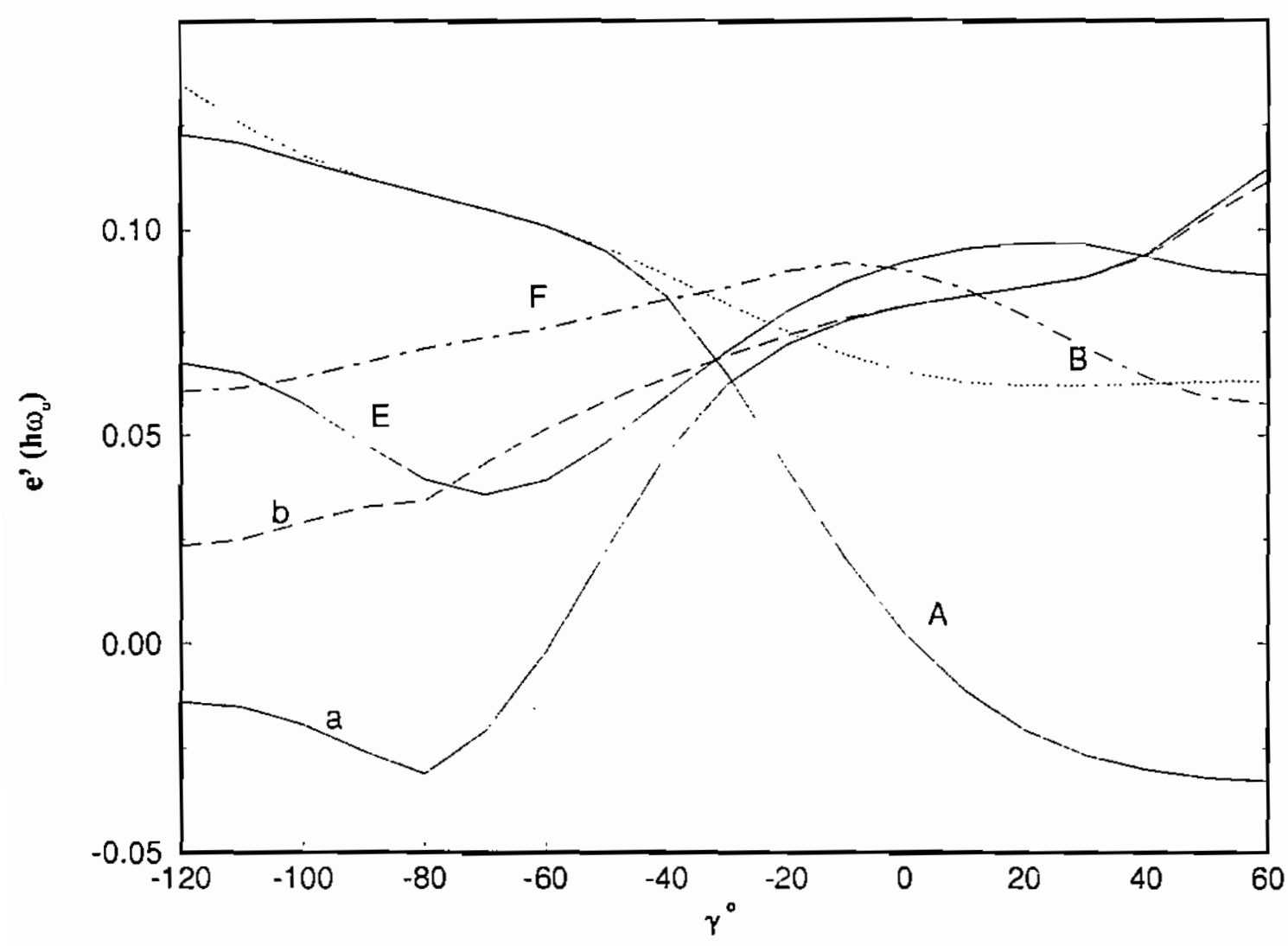

Figura 4.10: Valores dos routhianos dos primeiros estados de quasi-partículas calculados em função de $\gamma$ para prótons $(\mathrm{A}, \mathrm{B})$ e nêutrons $(\mathrm{a}, \mathrm{b})$ de configuração $h_{11 / 2}$ e prótons $(\mathrm{E}, \mathrm{F})$ de configuração $g_{7 / 2}$. Os parâmetros utiiizados nos cálculos foram $\beta=0.14, \Delta \approx 1$ $\mathrm{MeV}$ e $\hbar \omega=0.25 \mathrm{MeV}$. 
Cálculos de $E_{\text {tot }}^{\prime}$ para a banda $\pi[411] 1 / 2 \otimes \nu h_{11 / 2}$ (banda 3) mostram um mínimo em torno de $\gamma_{2} \approx-80^{\circ}$ oblata (figura 4.12). Já para a banda de 4 quasi-partículas observamos que o mínimo de $E_{\text {tot }}^{\prime}$ está em torno de $\gamma \approx 10^{\circ}$ e $\gamma \approx 5^{\circ}$ (figura 4.13) com uma deformação prolata para esta banda. Pode-se observar ainda que os mínimos das combinaçōes da "signature" favorecida e desfavorecida do próton com as do nêutron, isto é, as curvas [EaAB - EbAB], [FaAB - FbAB]) estão separadas apenas por $200 \mathrm{keV}$. Experimentalmente, isto é visto nas bandas $2 a$ e $2 b$, onde a separaçāo energética entre elas é desta ordem. Deste modo, a banda 2 a deve ser formada por [EaAB - EbAB] (com $K=3$ ) e a banda $2 \mathrm{~b}$ por $[\mathrm{FaAB}-\mathrm{FbAB}](\operatorname{com} K=8)$. Nos cálculos de $E_{\text {tot }}^{\prime}$ apresentados acima o ${ }^{136} \mathrm{Ce}(\mathrm{Z}=58, \mathrm{~N}=78)$, de forma oblata, foi tomado como caroço com $V_{p o}<0$.

Resumindo, podemos afirmar que o núcleo de ${ }^{138} \mathrm{Pr}$ é composto por 3 bandas (tabela 4.2), sendo que a banda semidesacoplada e a de 4 quasi-partícula mostram uma forma prolata, ligeiramente triaxial, enquanto que a banda 3, desacoplada, parece exibir forma oblata. Deste modo, os cálculos de CSM implicam na coexistência de forma em energias de excitaçōes bastante baixas.

Tabela 4.2: Resumo das configurações e valores de $\langle K\rangle$ para as bandas observadas no núcleo de ${ }^{138} \mathrm{Pr}$.

\begin{tabular}{|c|c|c|c|}
\hline Banda & $\langle K\rangle$ & configuração & forma \\
\hline 1 & 6 & $\pi h_{11 / 2} \otimes \nu h_{11 / 2}$ & prolata \\
\hline $2 \mathrm{a}$ & 3 & $\begin{array}{c}\pi[413] 5 / 2 \otimes \nu h_{11 / 2} \otimes\left(\pi h_{11 / 2}\right)^{2} \\
{[\mathrm{EaAB}-\mathrm{EbAB}]}\end{array}$ & prolata \\
\hline $2 \mathrm{~b}$ & 8 & $\begin{array}{c}\pi[413] 5 / 2 \otimes \nu h_{11 / 2} \otimes\left(\pi h_{11 / 2}\right)^{2} \\
{[\mathrm{FaAB}-\mathrm{FbAB}]}\end{array}$ & prolata \\
\hline 3 & 1 & $\pi[411] 1 / 2 \otimes \nu h_{11 / 2}$ & oblata \\
\hline
\end{tabular}




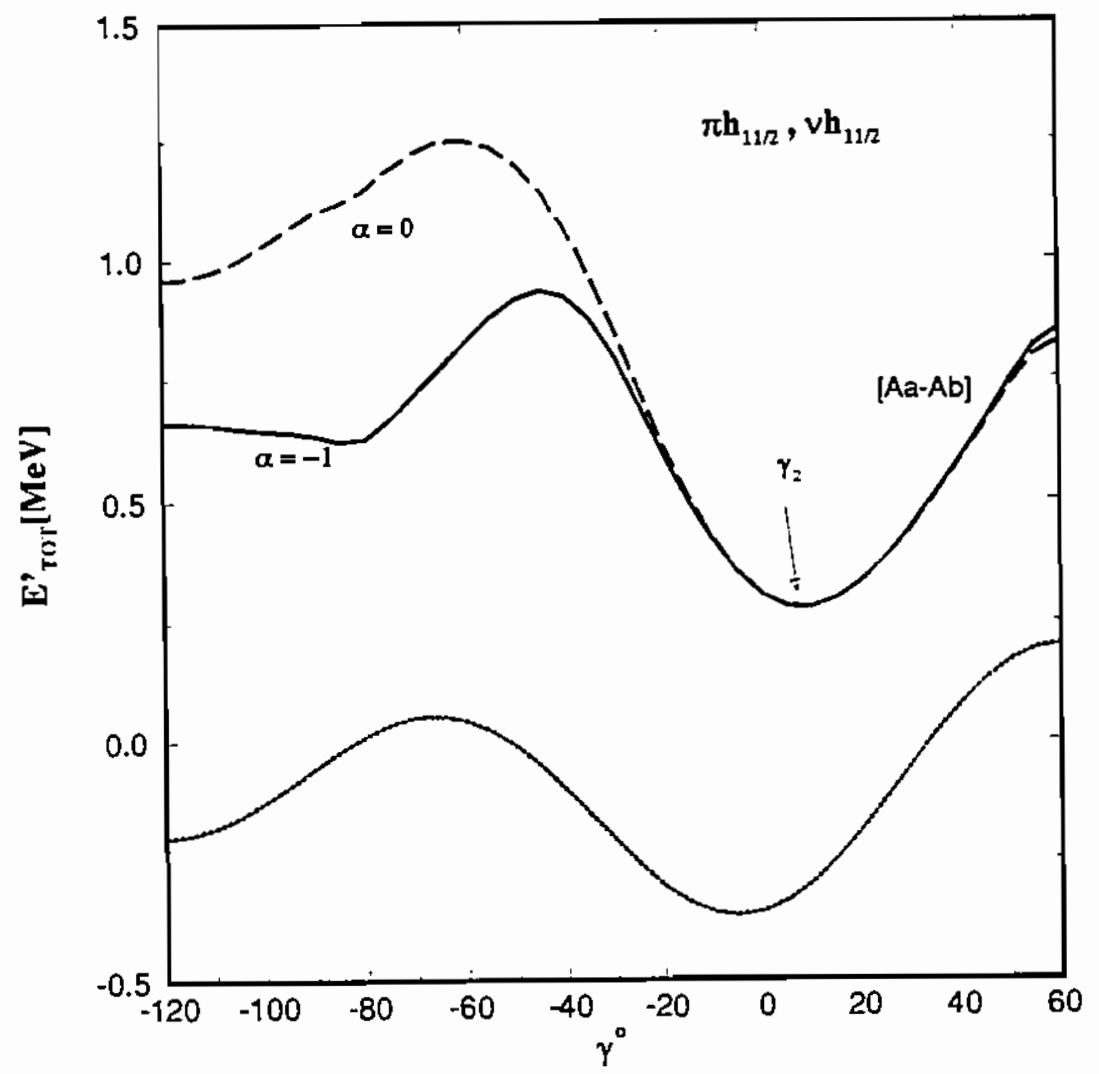

Figura 4.11: Routhiano total, $E_{\text {tot }}^{\prime}$, calculado em função da deformaçāo $\gamma$ para a configuraçāo $\pi h_{11 / 2} \otimes \nu h_{11 / 2}$ do ${ }^{138} \mathrm{Pr}$. Os cálculos foram feitos para a freqüência fixa $\hbar \omega=$ $0.03 \hbar \omega_{0}=0.25 \mathrm{MeV}$ e assumindo-se $\beta=0.14 ; \Delta \approx 1.0 \mathrm{MeV}$ e $V_{p o}=-0.4 \mathrm{MeV}$. A linha contínua é para $\alpha=-1$, a tracejada para $\alpha=0$ e a linha pontilhada se refere ao caroço. $\gamma_{2}$ é a deformação de equilibrio. 


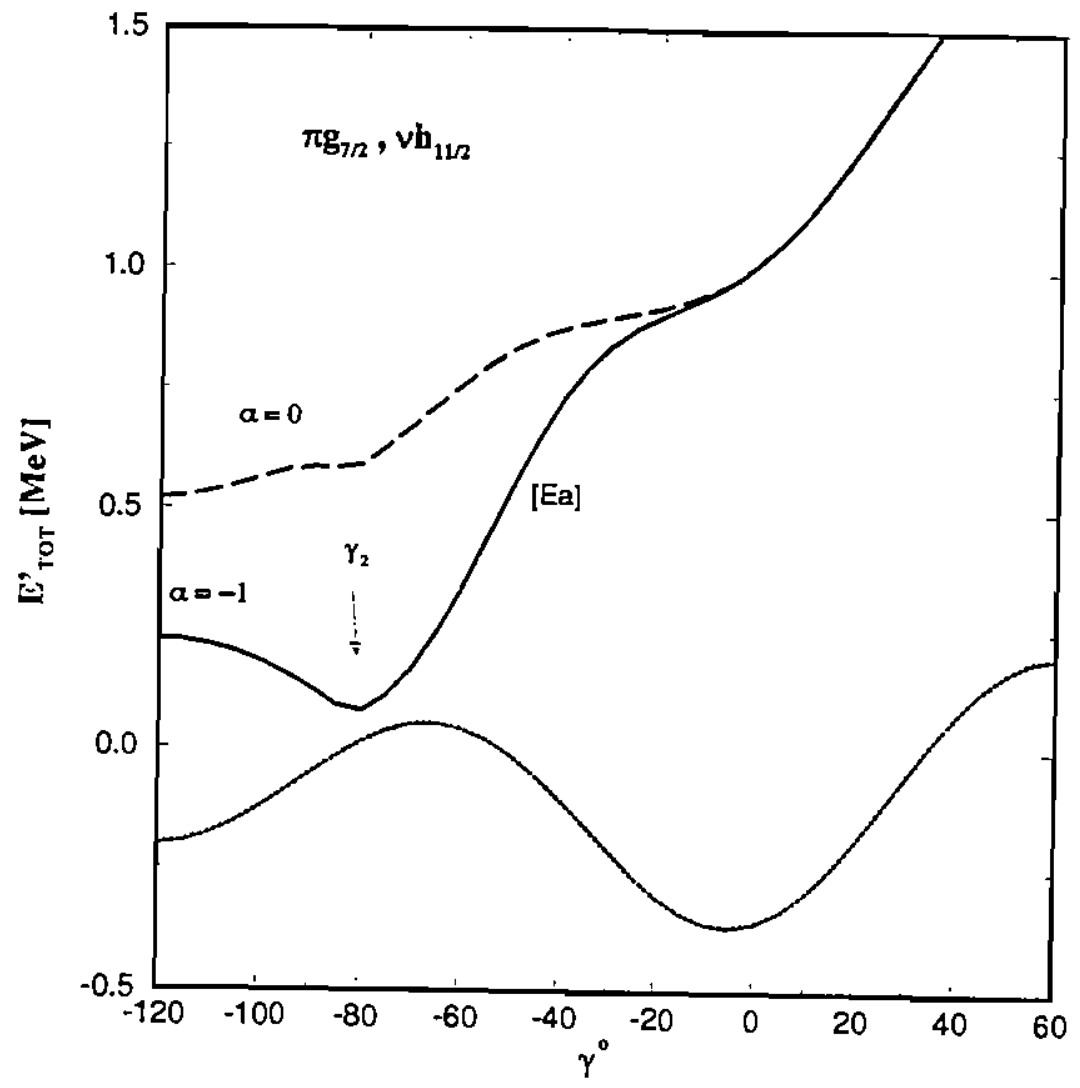

Figura 4.12: Routhiano total, $E_{\text {lot }}^{\prime}$, calculado em função da deformação $\gamma$ para a configuração $\pi[411] 1 / 2 \otimes \nu h_{11 / 2}$ do ${ }^{138} \operatorname{Pr}$. Os cálculos foram feitos para a freqüência fixa $\hbar \omega$ $=0.03 \hbar \omega_{0}=0.25 \mathrm{MeV}$ e assumindo-se $\beta=0.14 ; \Delta \approx 1.0 \mathrm{MeV}$ e $V_{p o}=-0.4 \mathrm{MeV}$. A linha contínua é para $\alpha=-1$, a tracejada para $\alpha=0$ e a linha pontilhada se refere ao caroço. $\gamma_{2}$ é a deformaçāo de equilibrio. 


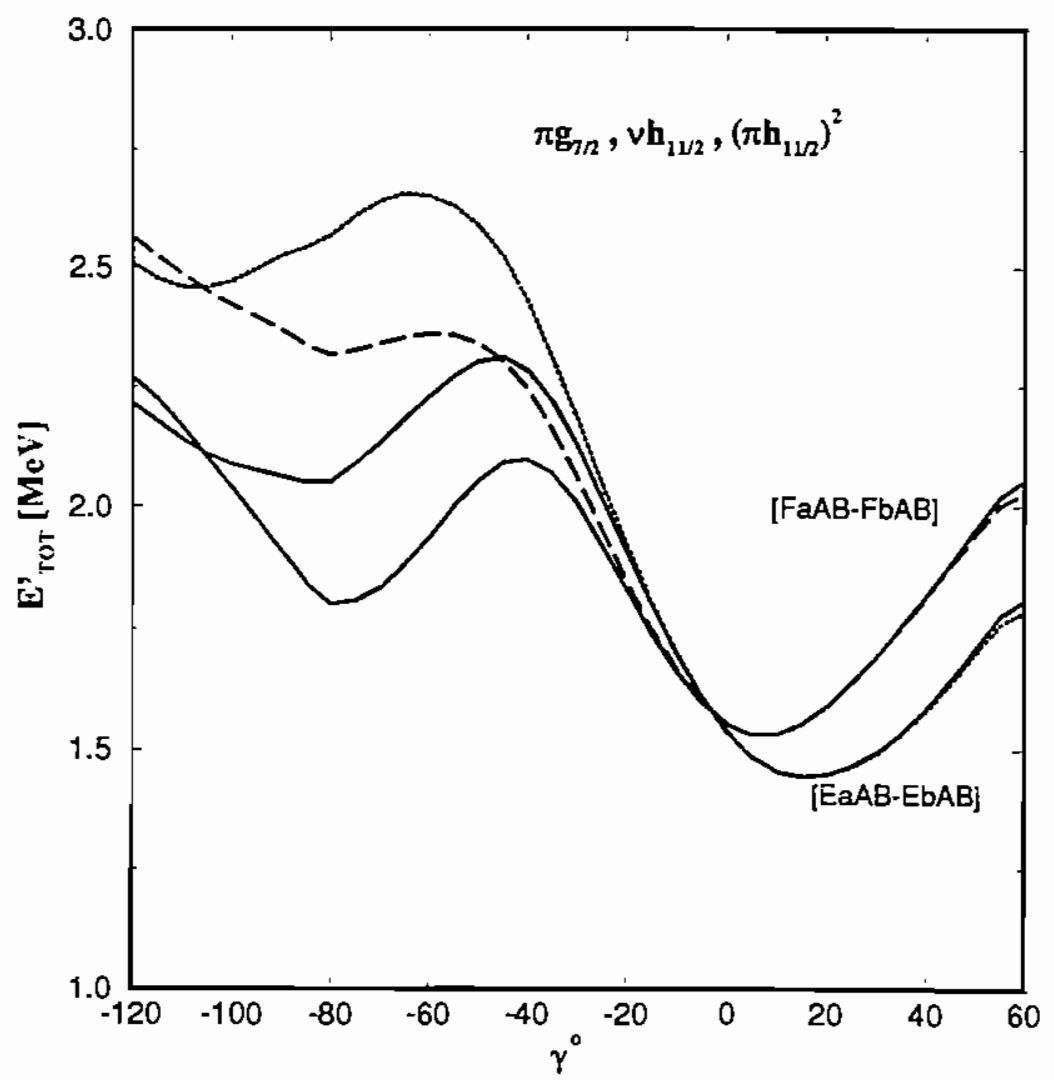

Figura 4.13: Routhiano total, $E_{t o t}^{\prime}$, calculado em função da deformaçâo $\gamma$ para a configuraçāo $\pi[413] 5 / 2 \otimes \nu h_{11 / 2} \otimes\left(\pi h_{11 / 2}\right)^{2}$ do ${ }^{138} \mathrm{Pr}$. Os cálculos foram feitos para a frequéncia fixa $\hbar \omega=0.03 \hbar \omega_{0}=0.25 \mathrm{MeV}$ e assumindo-se $\beta=0.14 ; \Delta \approx 1.0 \mathrm{MeV}$ \& $V_{p o}=-0.4 \mathrm{MeV}$. 


\subsection{Cálculos com PSM}

Foram realizados cálculos teóricos de "Projected Shell Model" para o núcleo ímparímpar de ${ }^{138} \mathrm{Pr}$ através do código ODOD desenvolvido por K. Hara e Y. Sun [15], conforme descriçāo da teoria feita na seção 2.2 . Em toda a regiāo de massa $A \approx 130$ - 140 considerouse um caroço inerte formado por 20 prótons e 40 nêutrons e foram consideradas três camadas principais para prótons e nêutrons, isto é, $\mathrm{N}=3,4$ e 5 . As constantes $G_{1}$ e $G_{2}$ do termo de emparelhamento da hamiltoniana (eq. 2.24 e 2.25) foram ajustadas para fornecer um valor de "pairing gap" em torno de 1.0 Mev, que está de acodo com a estimativa de $\Delta=135 /$ A, resultando em $G_{1}=19.12$ e $G_{2}=5.13$. A deformaçāo quadrupolar, $\epsilon_{2}=0.16$ foi ajustada de tal modo a reproduzir melhor as energias experimentais, enquanto que a deformação hexadecapolar, $\epsilon_{4}=0$ é tipica desta regiăo. 0 melhor valor encontrado para $\alpha$ (equação 2.26) foi de 0.20 que está de acordo com o valor sugerido na referência [25] para núcleos na regiāo de terras raras.

A comparação entre os resultados dos cálculos teóricos para vários parâmetros ajustados e os resultados experimentais para a banda 1 do núcleo de ${ }^{138} \mathrm{Pr}$ de configuração $\pi h_{11 / 2} \otimes \nu h_{11 / 2}$ é feita na figura 4.14. Os cálculos realizados para esta banda levam em conta que a partícula de valência ocupa a camada $N_{n}=5$ e $N_{p}=5$ (banda 55). A análise teórica desta banda yrast mostra que tanto os cálculos para deformações positivas como negativas, $\operatorname{com} \epsilon_{2}=|0.16|$ ajustam razoavelmente bem os dados experimentais. Esta possibilidade pode ser entendida lembrando-se que os níveis $h_{11 / 2}$ sāo quase que simétricos em relação à deformação nula e neste caso em específico os níveis de Fermi para nêutron e próton estão situados na parte superior e inferior desta subcamada. No entanto, quando os valores de energia correspondentes da banda 1 são plotados em função do spin (figura 4.15) observa-se que os cálculos teóricos para o valor positivo de deformação se aproximam mais dos dados experimentais. A análise do diagrama de bandas (figura 4.16) correspondente é importante para investigar a configuração da banda. E neste caso, em baixo spin, as configuraçōes dominantes são $\nu h_{11 / 2}(11 / 2) \otimes \pi h_{11 / 2}(1 / 2)$ e $\nu h_{11 / 2}(9 / 2) \otimes \pi h_{11 / 2}(3 / 2)$. Em spins mais altos observa-se que a configuraçāo $\nu h_{11 / 2}(7 / 2) \otimes \pi h_{11 / 2}(3 / 2)$ se torna 


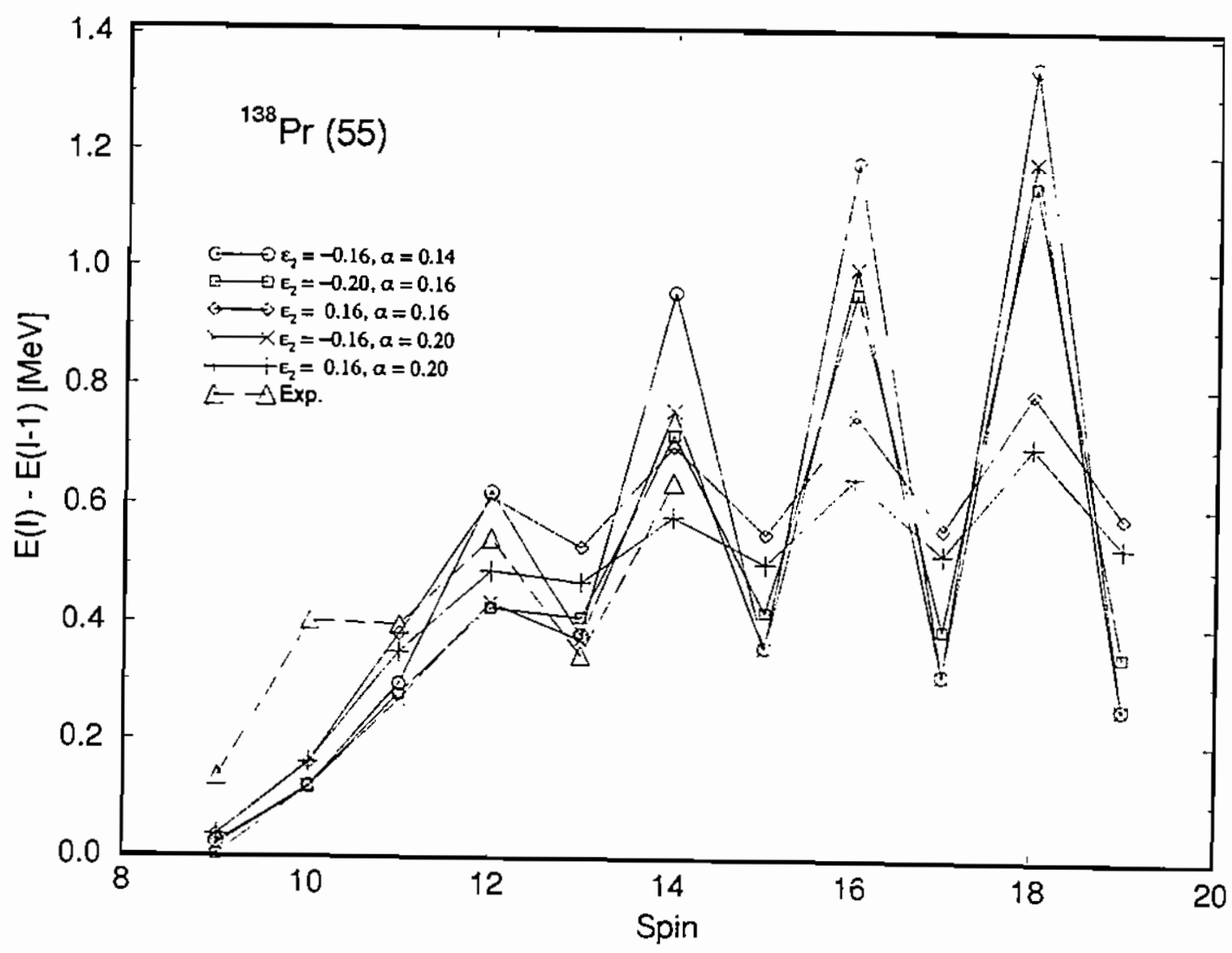

Figura 4.14: Cálculos teóricos para a banda 55 de ${ }^{138} \mathrm{Pr}$, para os vários parâmetros ajustados, em comparação com os pontos experimentais.

predominante. A linha grossa, que representa as energias da banda yrast depois da diagonalização da hamiltoniana, mostra um mínimo em torno de $8^{+}$que está de acordo com o spin inicialmente atribuido ao "band-head" da banda 1. As curvas suaves mostradas nesta figura, triângulos e asteriscos, correspondem a grandes valores de $K$; no entanto, se $K$ é pequeno e se cada urn ou ambos $K_{n}$ e $K_{p}$ são $1 / 2$ ou $3 / 2$, uma forma de "zig-zag" é observada. Esta característica é equivalente, no modelo de Bohr, ao efeito de desacoplamento e é o responsável pelo pequeno "staggering" na banda yrast. Infelizmente os dados experimentais param no spin 14 e somente indicam o "staggering" predito. 


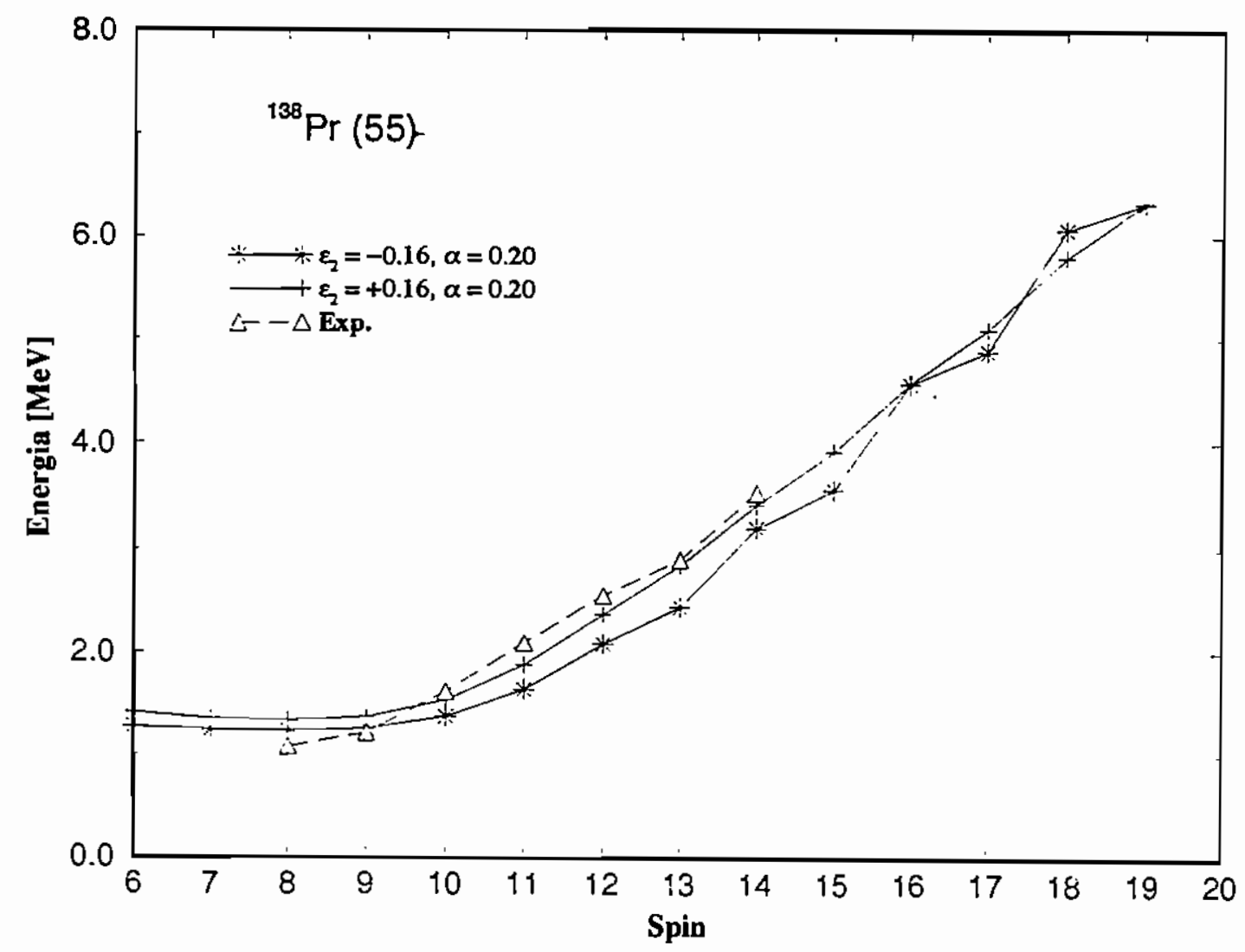

Figura 4.15: Energia versus spin dos dados experimentais da banda de configuração $\pi h_{11 / 2} \otimes \nu h_{11 / 2}$ de ${ }^{138} \operatorname{Pr}$ em comparação com os cálculos teóricos para $\epsilon_{2}= \pm 0.16$.

A análise teórica da banda 3 (banda 54) em comparação com os dados experimentais é mostrada na figura 4.17. Pode-se notar que os cálculos com deformação positiva possuem um acordo péssimo com os dados experimentais, enquanto que para $\epsilon_{2}=$ 0.16 a concordancia é ótima, sugerindo uma configuraçāo oblata para esta banda, isto é, $\left(\nu h_{11 / 2} \otimes \pi[411] 1 / 2\right)$. O spin do cabeça de banda iniciaimente atribuído, $9^{-}$, concorda com as previsões teóricas. 


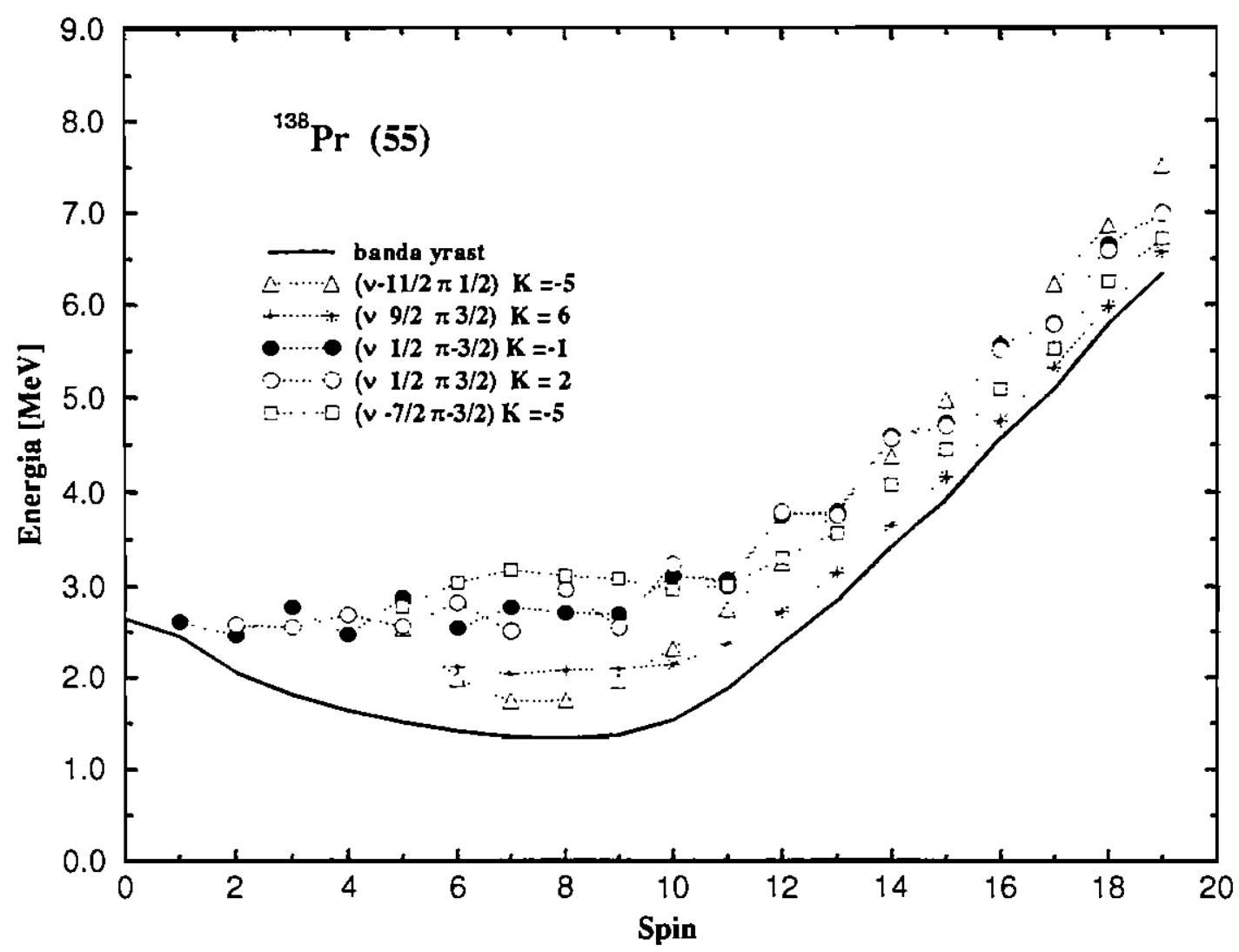

Figura 4.16: Diagrama de bandas para a configuração $55\left(\nu h_{11 / 2} \otimes \pi h_{11 / 2}\right)$ de ${ }^{138} \operatorname{Pr}$. Os valores grandes de $K$ pertencem à configuraçāo $\pi h_{11 / 2}$ e $\nu h_{11 / 2}$, enquanto que $\mathrm{K}=-1 \mathrm{e}$ 2 se referem ao orbital $f_{7 / 2}$ do nêutron.

Muitas das características desta banda 54 sāo mostradas no diagrama de bandas da figura 4.18, onde é enfatizado o caráter de "zigzag" das bandas com valor baixo de $K$. Como se pode observar nesta figura, nenhum valor alto de $K$ foi obtido, confirmando a atribuiçāo da configuração daàa acima. Os resultados dos cálculos ainda predizem que na banda 3 de ${ }^{138} \operatorname{Pr}$ (duplamente desacoplada) os estados com "signature" favorecida e desfavorecida são completamente degenerados, e esta degenerescência é muito difícil de ser verificada experimentalmente. 


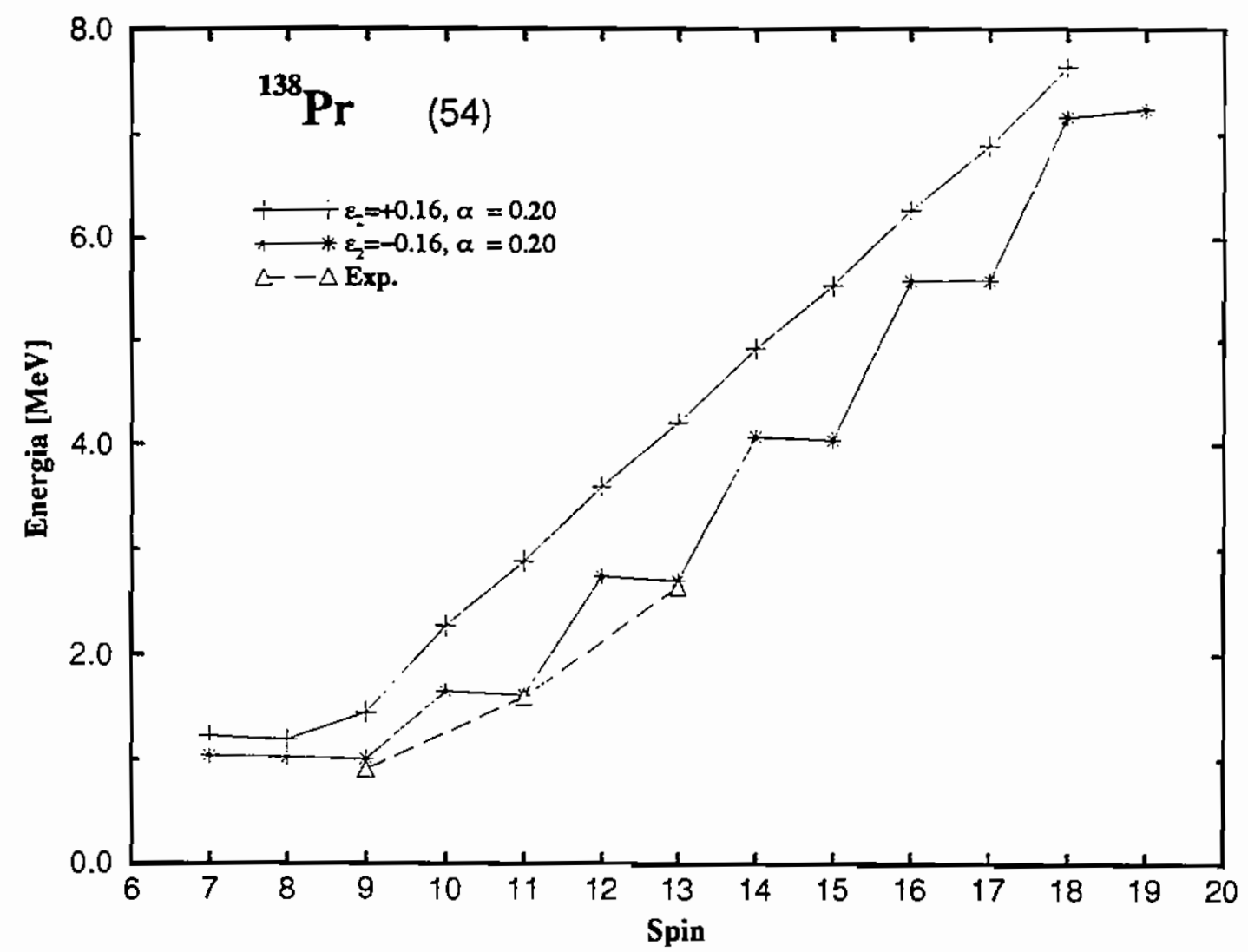

Figura 4.17: Níveis de energia teóricos para a deformação oblata $\left(\epsilon_{2}=-0.16\right)$ e para deformação prolata $\left(\epsilon_{2}=+0.16\right)$ plotados contra spins para a banda 54 do ${ }^{138} \operatorname{Pr}$ em comparação com os dados experimentais.

Dos resultados acima pode-se sugerir que a banda 1 de ${ }^{138} \mathrm{Pr}$ seja prolata com configuraçāo $\nu h_{11 / 2} \otimes \pi h_{11 / 2}$ e que a banda 3 de configuração $\nu h_{11 / 2} \otimes \pi[411] 1 / 2$ seja oblata, no entanto, parece ser pouco provável a coexistência de formas em energias de excitação tāo baixas [47]. Por outro lado, os cálculos acima da banda 55 não descartam a possibilidade de uma forma oblata, significando que tanto a banda positiva 55 como a banda negativa $\mathbf{5 4}$ podem ser descritas consistentemente assumindo-se uma forma obiata para o núcleo todo. 


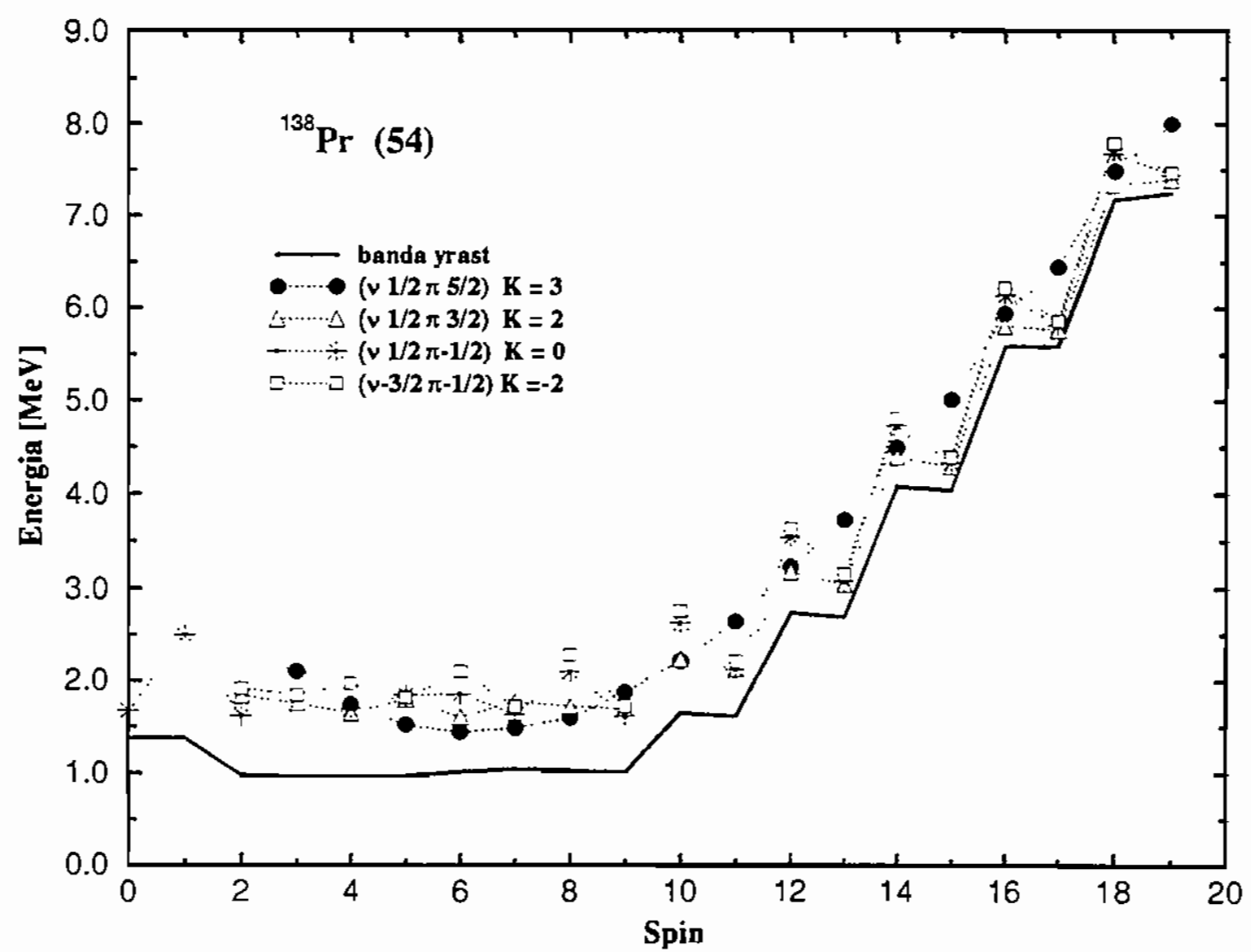

Figura 4.18: Diagrama de bandas para a configuraçāo 54 de ${ }^{138} \operatorname{Pr}$. Todas as projeções e os valores correspondentes de $K$ atribuídos pertencem à configuraçāo $\nu h_{11 / 2} \otimes \pi g_{7 / 2}$.

Deve-se salientar ainda que as outras bandas, 44 e $4 \bar{s}$, calculadas teoricamente nāo ajustaram os dados da banda $2(2 \mathrm{a}$ e $2 \mathrm{~b})$ de ${ }^{138} \mathrm{Pr}$, isto porque elas foram interpretadas como sendo bandas de 4 quasi-partículas e o código utilizado neste trabalho consegue trabalhar com no máximo 3 quasi-partículas. 


\section{Capítulo 5}

\section{Sistemática da Região de Massa}

\section{$\mathbf{A}=\mathbf{1 3 0}$}

Uma análise qualitativa dos dados experimentais da regiāo de $A \approx 130(Z=57-61$ e $\mathrm{N}=71$ - 79), mostra que existe uma sistemática nas bandas rotacionais observadas. Em primeiro lugar, nos núcleos com Z-ímpar e N-ímpar observa-se bandas yrast construídas sobre estados isoméricos, de configuraçāo $h_{11 / 2}$ que é o orbital intruso dos níveis de Nilsson. Por outro lado, nos núcleos duplamente ímpar observa-se bandas intensas semidesacopladas de configuração $\pi h_{11 / 2} \otimes \nu h_{11 / 2}$, típicas desta região.

Os isótonos, tanto para núcleos ímpares como ímpar-ímpar mostram bandas quase que idênticas, sugerindo que o próton é um mero espectador. No caso dos ímpares esta semelhança é vista na figura 4.3, onde fica clara a diferença de estrutura entre os núcleos de $\mathrm{N}=77$ e de $\mathrm{N}=79$. Em $\mathrm{N}=77$ as bandas observadas são fortemente acopladas, com seqüência $\Delta I=1$. Já para $N=79$, os núcleos apresentam as bandas $\nu h_{11 / 2} \operatorname{com}$ características desacopladas, $\Delta I=2$. Há um consenso na literatura de que os núcleos ímpares nesta região de massa mudam de forma quando os nêutrons se aproximam da camada fechada $N=82$, passando da deformação prolata em $N \leq 75$ para a forma oblata em N $=79$. 


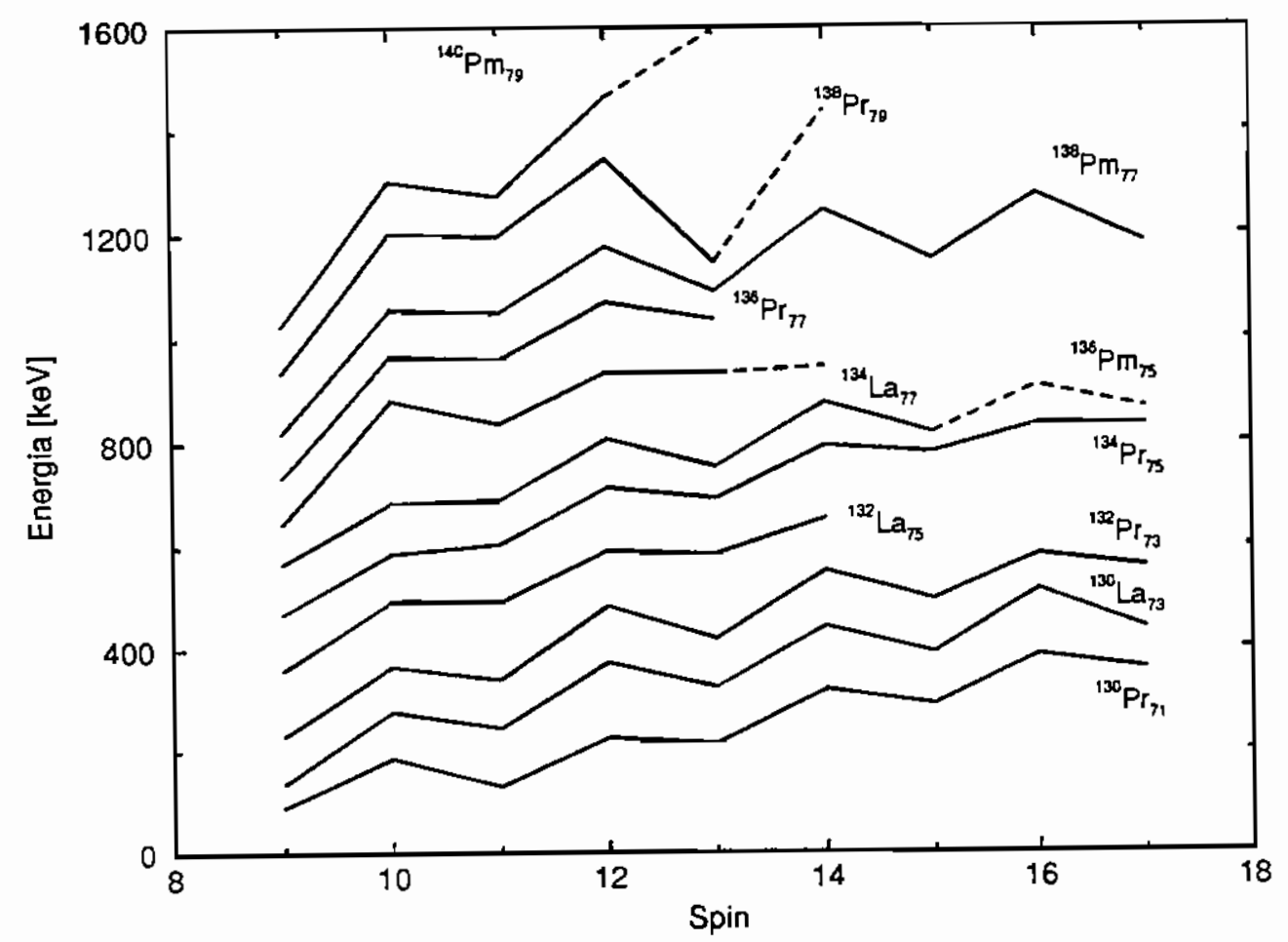

Figura 5.1: Comparação das energias das transições com $\Delta I=1$ das bandas $\pi h_{11 / 2} \otimes$ $\nu h_{11 / 2}$ dos núcleos ímpar -impar da região de massa $A \approx 130$ em função do spin. Cada uma das curvas foi afastada da anterior por um valor constante de $100 \mathrm{keV}$ para ficar clara a disposição dos dados experimentais.

Já os isótopos desta regiāo com Z $=57,59$ e 61, apresentados na figura 5.1 em forma de níveis de energia da banda $\pi h_{11 / 2} \otimes \nu h_{11 / 2}$ em funçāo do spin mostram, além do aumento no espaçamento entre os níveis com $N$ (vide seção 4.1), a semelhança entre os isótonos evidenciando ainda mais o comportamento passivo do próton.

Finalmente, para os núcleos impar-ímpar, além da banda principal observa-se bandas secundárias que mudarn de característica à medida que $\mathrm{N}$ aumenta. Assim, para $\mathrm{N}<75$ tem-se bandas $\pi h_{11 / 2} \otimes \nu g_{7 / 2}$ com interisidades equivalentes à banda yrast. Para $N>\pi j$ 
a situaçăo é um pouco mais complexa, pois começam a surgir nestes núcleos bandas de 4 quasi-partículas como é observado em ${ }^{134} \mathrm{Pr}{ }^{136,138} \mathrm{Pm}$ e ${ }^{138} \mathrm{Pr}$, figura 4.5 , onde há indicações de mudança e até mesmo de coexistência de forma. No caso de $\mathrm{N}>79$, a banda $\pi h_{11 / 2} \otimes \nu h_{11 / 2}$ começa a ficar diferente em relaçāo aos núcleos anteriores, a tal ponto que sua intensidade fica concentrada nos três primeiros núveis e começam a surgir estados de partícula independente e não mais estruturas que representam o sistema coletivo, como pode-se observar nos núcleos de ${ }^{140} \mathrm{Pm}$ e ${ }^{141} \mathrm{Pm}$.

A partir dos dos dados experimentais expostos acima, se pode observar que não só os núcleos ímpares mas também os duplamente ímpares sofrem uma mudança de forma ao redor de $\mathrm{N}=78$.

\subsection{Sistemática com CSM}

Apesar da região de massa $A \approx 130$ ter sido estudada essencialmente por três grupos experimentais - Liverpool, Stony Brook e Pelletron - e os dados terem sido interpretados com CSM, foram refeitos novamente estes cálculos de CSM para esta região padronizandose os parâmetros, $\beta, \Delta$, etc., segundo os critérios usados no capítulo 2 .

Em primeiro lugar, será examinado o efeito da força polarizadora das diferentes quasipartículas em função do nêutron. Os cálculos dos routhianos de quasi-partículas dos nêutrons no orbital $h_{11 / 2}$ em função da deformação gama para os isótopos de $\operatorname{Pr}$ (figura 5.2) mostram uma força polarizadora forte que tende para valores mais negativos de $\gamma$, passando de triaxial $\gamma \approx-50^{\circ}\left({ }^{132} \mathrm{Pr}, \mathrm{N}=73\right)$, pelo oblato $\gamma \approx-70^{\circ}\left({ }^{136} \mathrm{Pr}, \mathrm{N}=77\right)$ para o triaxial tendendo ao oblato não coletivo $\gamma \approx-80^{\circ}\left({ }^{138} \mathrm{Pr}, N=79\right)$. Estes mínimos profundos vão se tornando mais suaves à medida que $N$ se aproxima da camada fechada. Figuras semelhantes foram obtidas para os núcleos de La e Pm.

Já nos cálculos dos prótons no orbital $h_{11 / 2}$, figura 5.3 , a força polarizadora é igualmente forte, mas no sentido oposto, isto é, para formas prolatas, como também para os núcleos de La e Pm. 


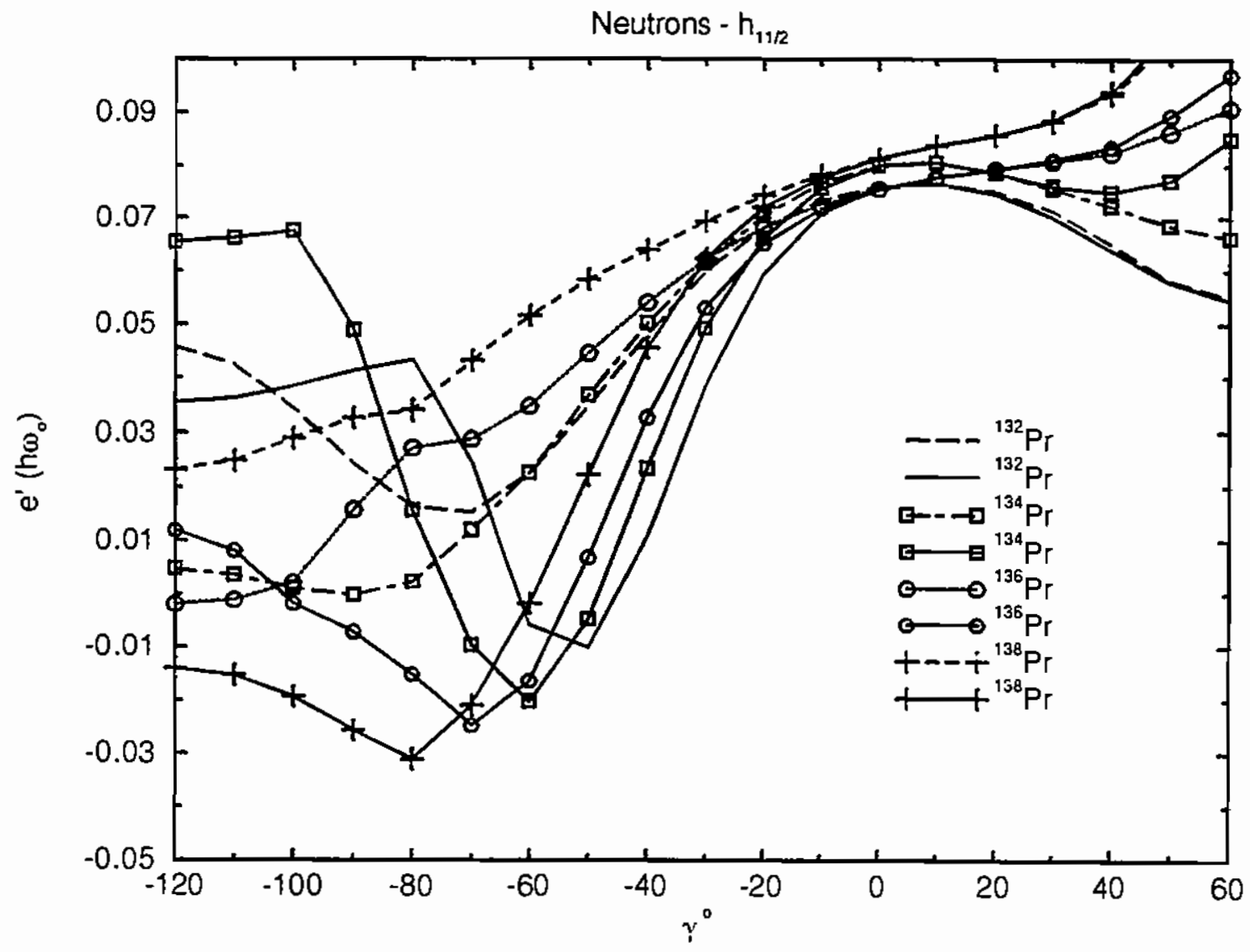

Figura 5.2: Comparação dos vários cálculos teóricos de e' $\mathrm{x} \gamma$ do orbital $h_{11 / 2}$ do nêutron para os isótopos ímpar-ímpar de $\operatorname{Pr}$ da região de massa $A \approx 130-140$. Os cálculos foram realizados para $\hbar \omega=0.03 \hbar \omega_{0} ; \Delta=0.13 \hbar \omega_{0} ; \beta=0.22,0.20,0.16$ e 0.14 e $\lambda_{n}=5.96$, $6.02,6.07$ e $6.10 \hbar \omega_{0}$ para os núcleos de ${ }^{132,134,136} \mathrm{Pr}$ e ${ }^{138} \mathrm{Pr}$, respectivamente.

Cálculos similares feitos para o orbital $g_{7 / 2}$ do próton dos núcleos ímpar-ímpar desta região, mostram um mínimo raso que permanece em torno de $\gamma=-60^{\circ}$ e se torna mais suave à medida que $\mathrm{N}$ cresce, figura 5.4, indicando um efeito muito menor na polarizaçāo para a forma oblata. 


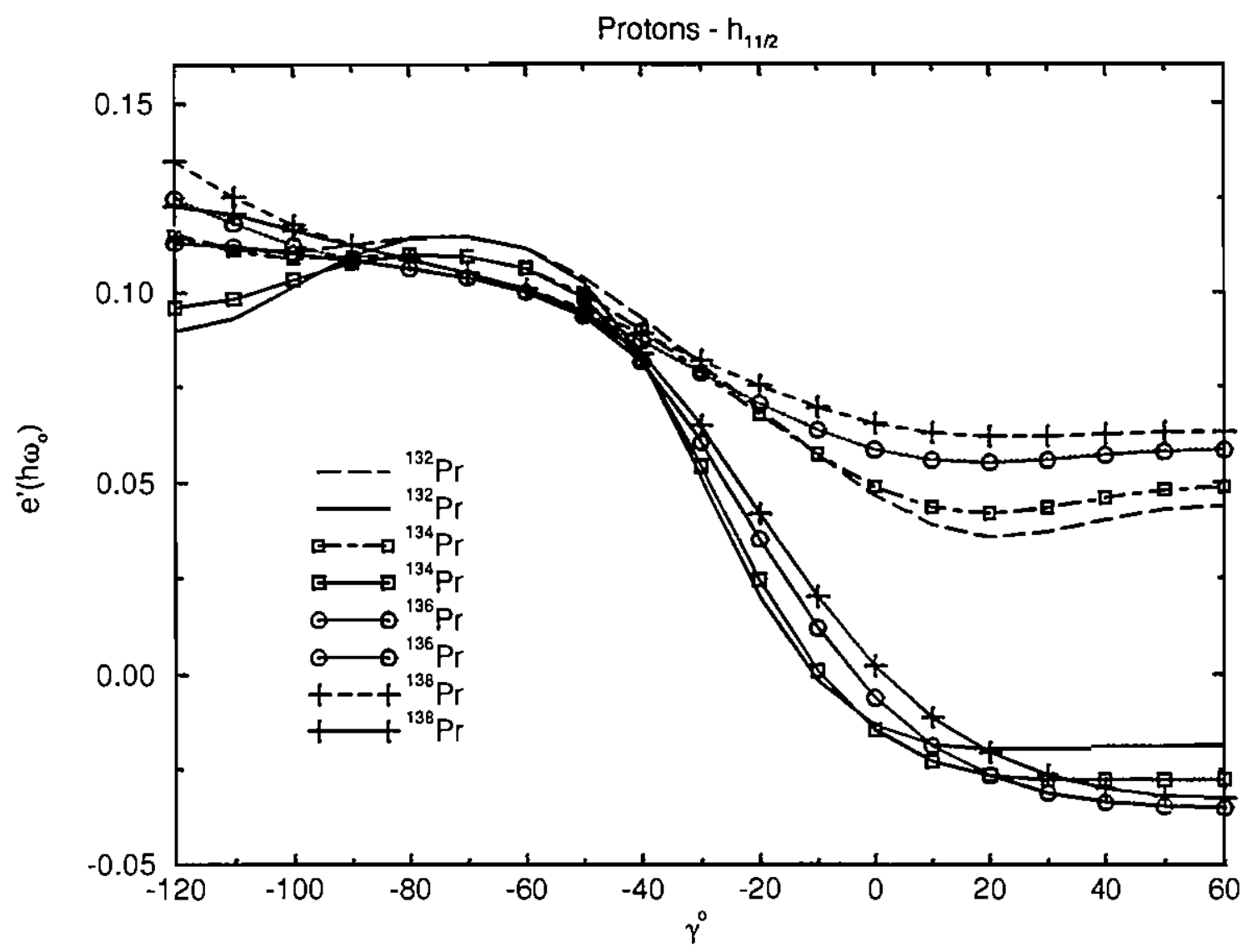

Figura 5.3: Comparaçāo dos vários cálculos teóricos de e' $\mathrm{x} \gamma$ do orbital $h_{11 / 2}$ do próton para os isótopos ímpar-impar de Pr da regiāo de massa $A \approx 130$ - 140. Os cálculos foram realizados para $\hbar \omega=0.03 \hbar \omega_{0}, \Delta=0.13 \hbar \omega_{0}, \beta=0.22,0.20,0.16$ e 0.14 e $\lambda_{n}=5.58$, $5.59,5.61$ e $5.62 \hbar \omega_{0}$ para os núcleos de ${ }^{132,134,136} \operatorname{Pr}$ e ${ }^{138} \operatorname{Pr}$ respectivamente.

Como mencionado na seçāo 2.1.1, no cálculo de $E_{T O T}^{\prime}$, ievamos em conta as contribuições dos routhianos das quasi-partículas, próton e nêutron, e o efeito do caroço que se reflete no sinal e na magnitude do coeficiente $V_{p o}$, que depende ainda do núcleo- caroço escolhido. A análise feita aqui considerou o núcleo par-par mais próximo, Z-1 e N-1, como sendo o caroço, que para $\mathrm{Z} \leq 61$ e $\mathrm{N} \leq 79$ tipicamente possuem formas prolatas e assim prevalece esta forma para quase todas as bandas observadas nos núcleos ímpar-ímpar. No 


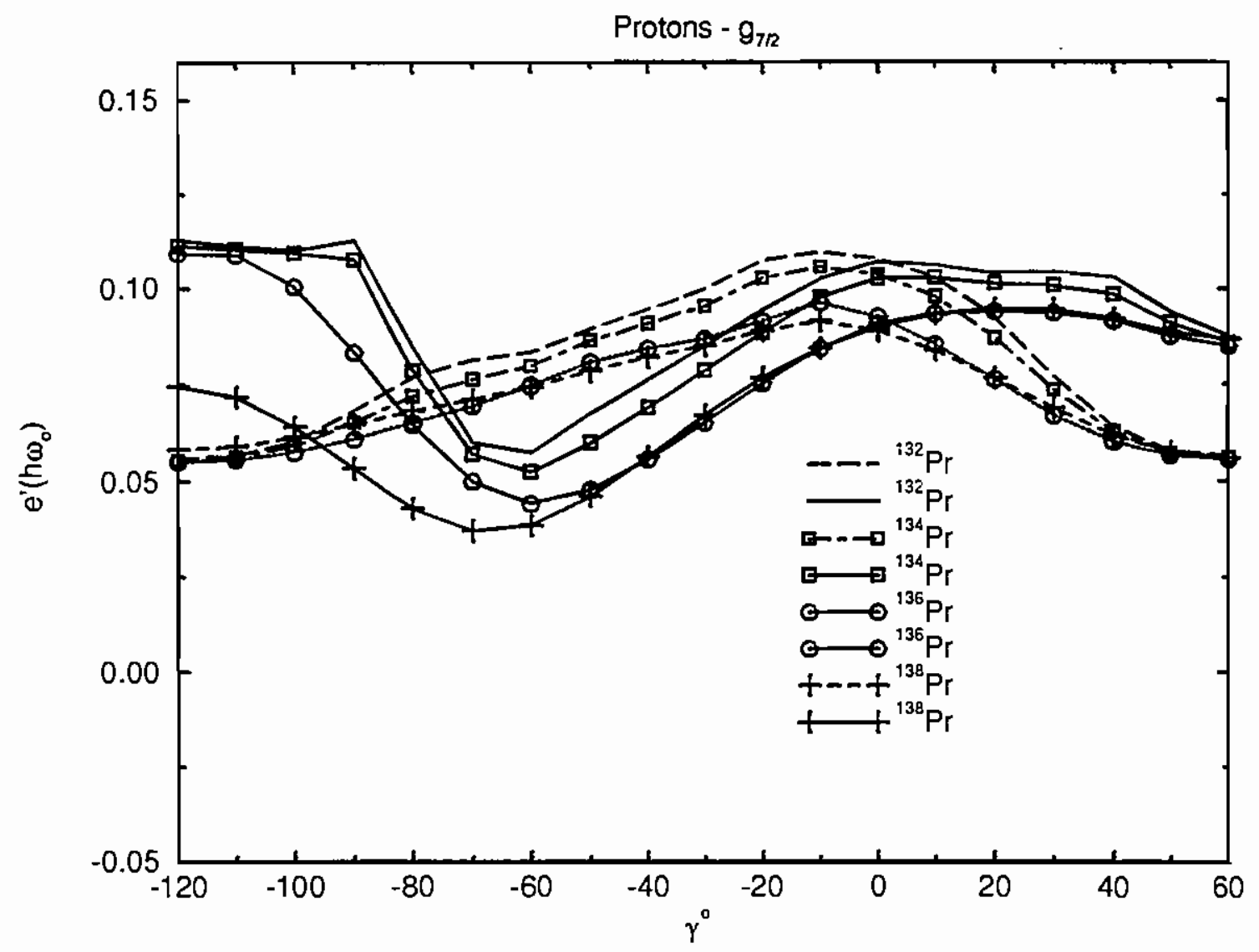

Figura 5.4: Comparação dos vários cálculos teóricos de e' x $\gamma$ do orbital $g_{7 / 2}$ do próton para os isótopos ímpar-ímpar de Pr da regiāo de massa $A \approx 130$ - 140. Os cálculos foram realizados para $\hbar \omega=0.03 \hbar \omega_{0}, \Delta=0.13 \hbar \omega_{0}, \beta=0.22,0.20,0.16$ e 0.14 e $\lambda_{n}=5.58$, $5.59,5.61$ e $5.62 \hbar w_{0}$ para os núcleos de ${ }^{132,134,136} \operatorname{Pr}$ e ${ }^{138} \operatorname{Pr}$, respectivamente.

caso do ${ }^{138} \mathrm{Pr}$, na banda $\pi g_{7 / 2} \otimes \nu h_{11 / 2}$ onde as forças polarizadoras tanto do próton como do nêutron, que tendem para $\gamma \approx-60^{\circ}$, prevalecem sobre o caroço e resultam na forma final oblata para esta configuraçāo. Na figura 5.5 são ilustrados os efeitos do sinal e da magnitude de $V_{p o}$ sobre $E_{T O T}^{\prime}$ para a banda $\pi h_{11 / 2} \otimes \nu h_{11 / 2}$ e para $\pi g_{7 / 2} \otimes \nu h_{11 / 2}$. Pode-se observar ainda, que se for utilizado como caroço o núcleo de ${ }^{140} \mathrm{Nd}, \mathrm{Z}+1$ e $\mathrm{N}+1$, que é oblato, a banda $\pi h_{11 / 2} \otimes \nu h_{11 / 2}$ também seria oblata. 
a)

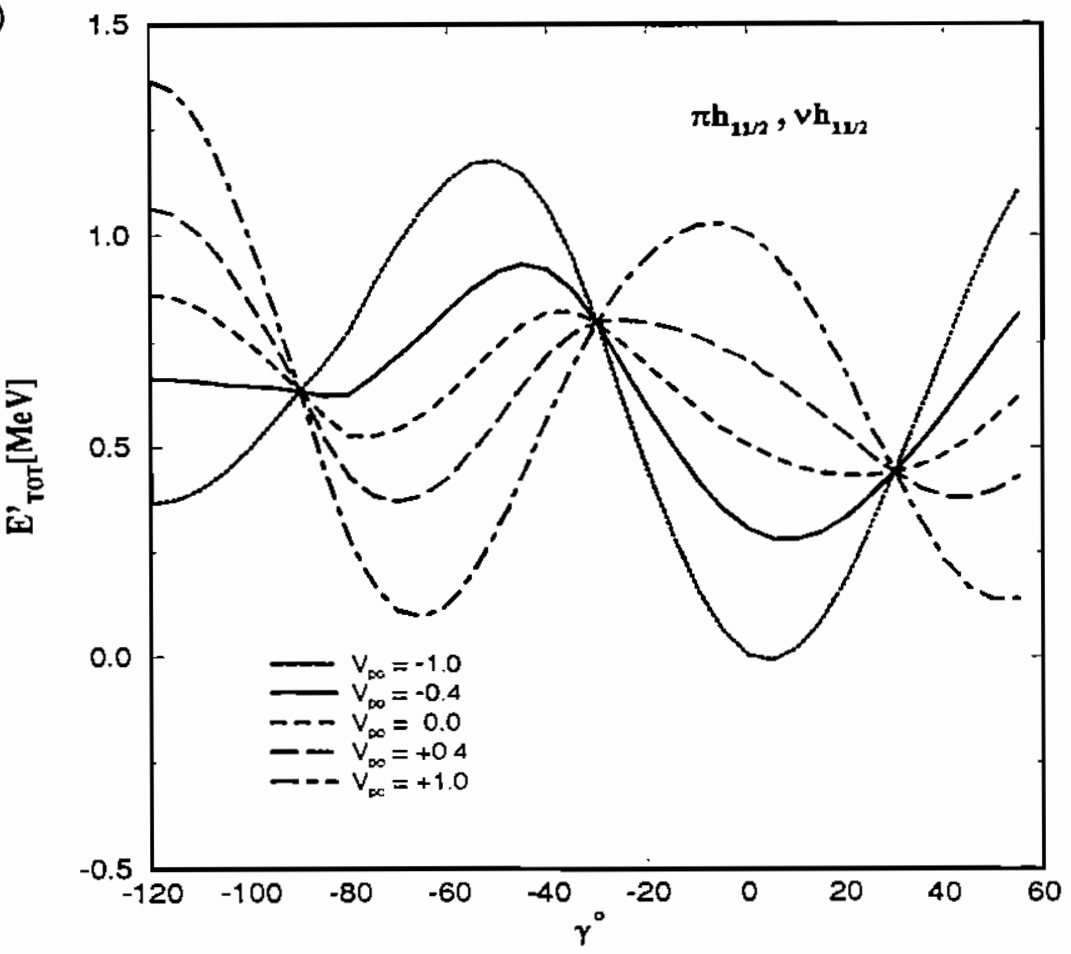

b)

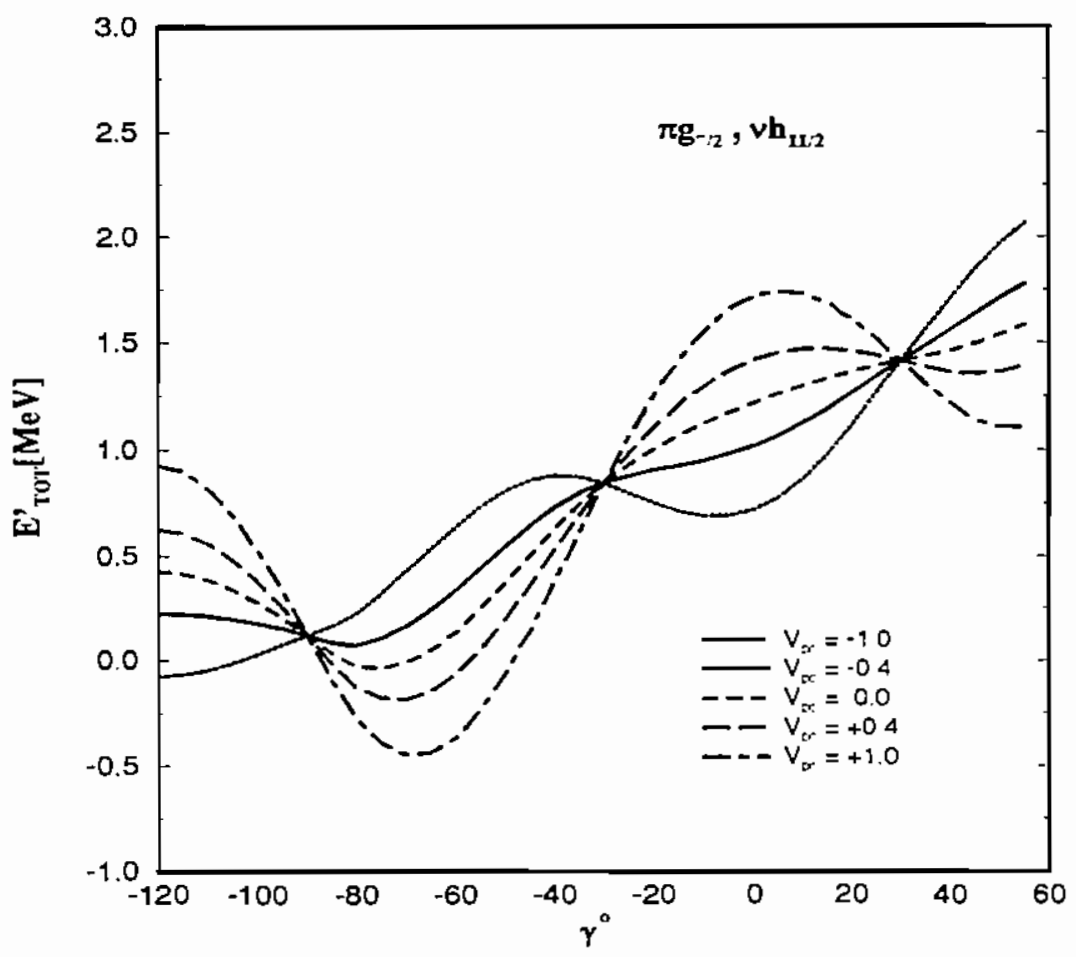

Figura 5.5: Cálculos de $E_{T O T}^{\prime}$ versus $\gamma$ para a banda a) $\pi h_{11 / 2} \otimes \pi h_{11 / 2}$ e b) $\pi g_{7 / 2} \otimes \pi h_{11 / 2}$ do ${ }^{138} \operatorname{Pr}$ com diferentes valores de $V_{p o}$. 
Os cálculos sistemáticos de routhiano total (seção 2.1) realizados para a banda $\pi h_{11 / 2} \otimes$ $\nu h_{11 / 2}$ dos núcleos duplamente ímpar da refião $\mathrm{A} \approx 130-140\left(V_{p o}=-0.4\right)$, mostram que o mínimo da energia $E_{T O T}^{\prime}$ se desloca de $\gamma \approx-5^{\circ}$ até $\gamma \approx 15^{\circ}$ (chamaremos estes gamas da deformação de equilíbrio de $\gamma_{2}$ ), indicando uma ligeira triaxialidade à medida que o número de nêutrons aumenta (figura 5.6). Os valores de "signature splitting" sâo pequenos, da ordem de 0 a $10 \mathrm{keV}$, muito menores do que os dados experimentais, que estāo em torno de $50 \mathrm{keV}$, menos para ${ }^{138} \mathrm{Pr}$. Para reproduzir o valor experimental seria necessário usar valores de $\gamma$ bem maiores. A deformação $\gamma$ para os correspondentes valores de $\Delta e_{e x p}^{\prime}$ tirados dos gráficos de e' $\mathrm{x} \gamma$ (que chamaremos de $\gamma_{1}$ ) estão em torno de $\approx-25^{0}$ (tabela 5.1) indicando deformação triaxial. Esta discrepância entre os cálculos de $E_{T O T}^{\prime}$ e os dados experimentais também foi observada nos núcleos ímpares [46] e as causas não são claras; talvez a falha esteja no modelo fenomenológico de Frauendorf e May [21] onde a contribuição do caroço tem uma forma muito simplificada

Tabela 5.1: Valores de "signature splitting" experimental e teórico e os correspondentes valores de gama, $\gamma_{1}$ e $\gamma_{2}$ respectivamente, para as bandas de configuração $\pi h_{11 / 2} \otimes \nu h_{11 / 2}$, dos núcleos da região de massa $\mathrm{A} \approx 130-140$.

\begin{tabular}{|c|c|c|c|c|c|}
\hline Núcleo & Ref. & $\Delta e_{\text {exp }}^{\prime}(\mathrm{keV})$ & $\gamma_{1}$ & $\Delta e_{\text {teo }}^{\prime}(\mathrm{keV})$ & $\gamma_{2}$ \\
\hline${ }^{130} \mathrm{La}$ & {$[14]$} & 50 & $-20^{\circ}$ & 5 & $0^{\circ}$ \\
${ }^{132} \mathrm{La}$ & {$[4]$} & 50 & $-25^{\circ}$ & 0 & $10^{\circ}$ \\
${ }^{134} \mathrm{La}$ & {$[3]$} & 50 & $-25^{\circ}$ & 0 & $15^{\circ}$ \\
${ }^{132} \mathrm{Pr}$ & {$[41]$} & 50 & $-15^{\circ}$ & 12 & $-5^{\circ}$ \\
${ }^{134} \mathrm{Pr}$ & {$[42,43]$} & 50 & $-25^{\circ}$ & 1 & $0^{\circ}$ \\
${ }^{136} \mathrm{Pr}$ & {$[3]$} & 40 & $-25^{\circ}$ & 0 & $15^{\circ}$ \\
${ }^{138} \mathrm{Pr}$ & pres. trab. & 0 & $0^{\circ}$ & 0 & $10^{\circ}$ \\
\hline
\end{tabular}

No caso das bandas secundárias, os cálculos de $E_{T O T}^{\prime}$ resultam na forma prolata para os núcleos com $\mathrm{N} \leq 73$. No caso da banda de 4 quasi-partículas do ${ }^{138} \operatorname{Pr}(\mathrm{N}=79)$ tem-se que para valores negativos de $V_{p o}$ (caroço prolato) esta banda é prolata; no entanto, para 

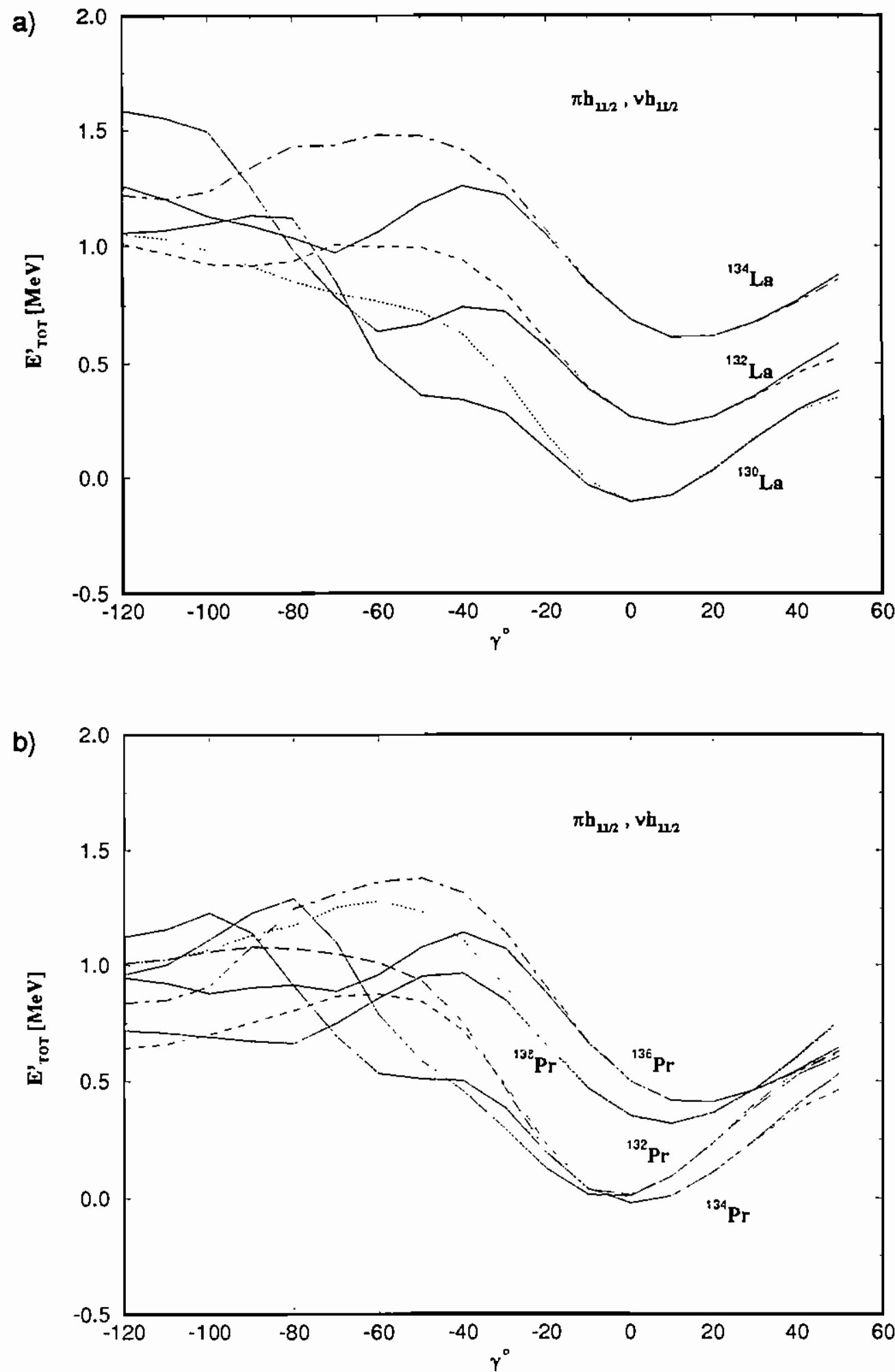

Figura 5.6: Cálculos sistemáticos de $E_{T O T}^{\prime}$ versus $\gamma$ para a banda $\pi h_{11 / 2} \otimes \pi h_{11 / 2}$ dos núcleos duplamente ímpar da região de massa $A \approx 130$, a) isótopos de La e b) isótopos de Pr. 
valores positivos de $V_{p o}$ (caroço oblato) ela fica oblata com mínimo em torno de $-60^{\circ}$ conforme figura 5.7 , mas com um "signature splitting" muito grande $\approx 500 \mathrm{keV}$, que não é consistente com os dados experimentais. Conclui-se então que a banda de 4 quasipartículas do ${ }^{138} \operatorname{Pr}$ deve ser prolata.

Um caso curioso são os núcleos isótonos de $N=77$. O núcleo de ${ }^{138} \mathrm{Pm}(\mathrm{N}=77)$ mostra um banda que, para baixos spins composta por apenas transiçōes E2 fracas com configuração atribuída de $\pi[413] \otimes \nu h_{11 / 2}$. Para $I>11 \hbar$ ela possui transiçōes mais fortes, de energia de $\approx 200 \mathrm{keV}$ e natureza dipolar (M1), muito similar à banda de 4 quasi-partículas dos núcleos vizinhos de ${ }^{134,138} \mathrm{Pr}$ e ${ }^{136} \mathrm{Pm}$ (vide figura 4.5). Segundo os autores [44], a força de polarizaçāo do próton no orbital $g_{7 / 2}$ e do nêutron em $h_{11 / 2}$ tende a valores de $\gamma \approx-65^{\circ}$ e isto leva ao núcleo oblato para a parte de baixos spins desta banda. Depois do cruzamento, a força de polarização forte do próton $h_{11 / 2}$ prevalece novamente e induz a uma forma prolata $\left(\gamma \approx 0^{\circ}\right)$, resultando assim em coexistência de formas na mesma banda, sendo coerente com os cálculos de $E_{T O T}^{\prime}$ das figuras 5.5 e 5.6 para $V_{p o}=-$ 0.4. Estes núcleos próximos à camada fechada são quase que esféricos e então qualquer variação pequena devido às forças de polarizaçāo pode levar o núcleo a mudar de forma.

\subsection{Sistemática com PSM}

Foram realizados cálculos teóricos com o novo modelo de camadas com projeção de momento angular (PSM) para os núcleos ímpar-ímpar e ímpares da regiāo de massa $\mathrm{A} \approx 130$ 140, e os parâmetros ajustados encontram-se na tabela 5.2 .

$\mathrm{Na}$ discussão seguinte são apresentados os resultados obtidos com o modelo PSM. A discussāo será feita na tentativa de explicá-los no contexto da sistemática desta regiāo, e para isto os resultados serão divididos em três grupos: 


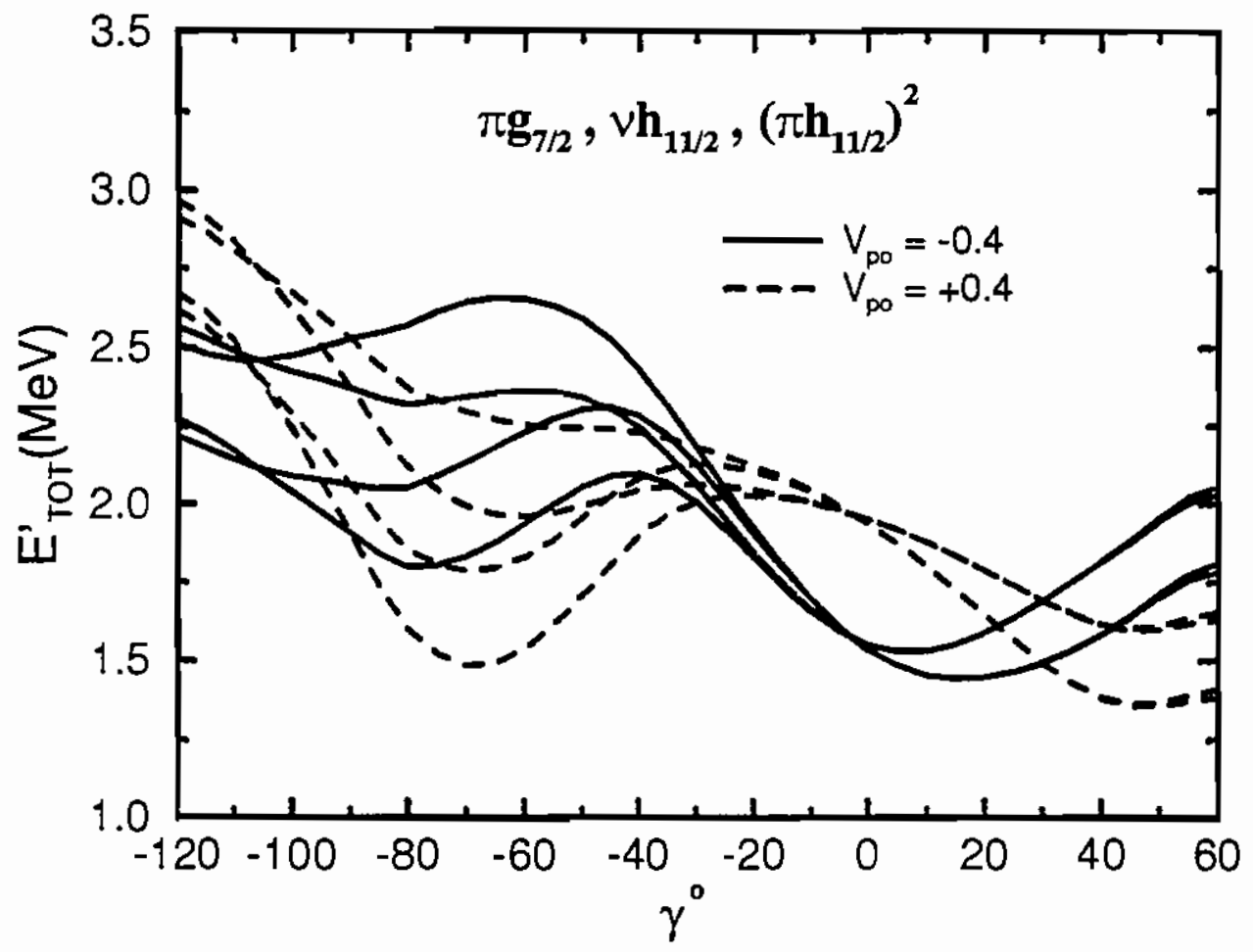

Figura 5.7: Cálculos de $E_{T O T}^{\prime}$ versus $\gamma$ para a banda $\pi[413] 5 / 2 \otimes \nu \dot{h}_{11 / 2} \otimes\left(\pi h_{11 / 2}\right)^{2}$ do ${ }^{138} \operatorname{Pr}$ para $Y_{p o}=-0.4$ e 0.4 . 
Tabela 5.2: Parâmetros usados nos cálculos do modelo de PSM.

\begin{tabular}{|c|c|c|c|c|}
\hline Núcleo & $\alpha$ & $\begin{array}{c}\Delta_{n} \\
(\mathrm{MeV}\end{array}$ & $\begin{array}{c}\Delta_{p} \\
(\mathrm{MeV})\end{array}$ & $\epsilon_{2}$ \\
\hline${ }^{130} \mathrm{La}$ & 0.20 & 1.01 & 0.93 & +0.22 \\
${ }^{132} \mathrm{La}$ & 0.20 & 0.96 & 0.94 & +0.20 \\
${ }^{134} \mathrm{La}$ & 0.20 & 0.96 & 0.96 & +0.18 \\
${ }^{132} \mathrm{Pr}$ & 0.20 & 1.11 & 0.96 & +0.22 \\
${ }^{134} \mathrm{Pr}$ & 0.20 & 0.95 & 0.93 & +0.20 \\
${ }^{136} \mathrm{Pr}$ & 0.20 & 0.95 & 0.97 & +0.18 \\
& 0.20 & 0.96 & 1.03 & -0.18 \\
${ }^{138} \mathrm{Pr}$ & 0.20 & 0.86 & 1.01 & +0.16 \\
& 0.20 & 0.70 & 1.06 & -0.18 \\
\hline
\end{tabular}

a) $N \leq 75$

O núcleo de ${ }^{130} L a$ foi extensivamente estudado em vários laboratórios [48, 49, 50], inclusive no laboratório Pelletron [14]. A figura 5.8 mostra os resultados dos vários cálculos teóricos em comparação com os dados experimentais do presente núcleo para a banda de configuração $\nu h_{11 / 2} \otimes \pi h_{11 / 2}$ (banda 55). Através desta figura pode-se observar que o melhor ajuste se dá para a deformação $\beta=0.22$ e $\alpha=0.20$ que está de acordo com o valor da sistemática da região.

Na figura 5.9 temos os cálculos teóricos juntamente com os dados experimentais das referências [14] e $[49,50]$ para a banda $\nu h_{11 / 2} \otimes \pi h_{11 / 2}$ de ${ }^{130} L a$. Uma boa concordância foi obtida entre os dados do Laboratório Pelletron [14] e a teoria onde é reproduzido o "zig-zag" observado experimentalmente e é confirmado o spin $8^{+}$atribuído à cabeça de banda desta configuração. É necessário enfatizar que os dados do Laboratório Pelletron [14], Stony Brook [48] e Liverpool [49, 50] são essencialmente os mesmos no que diz respeito 


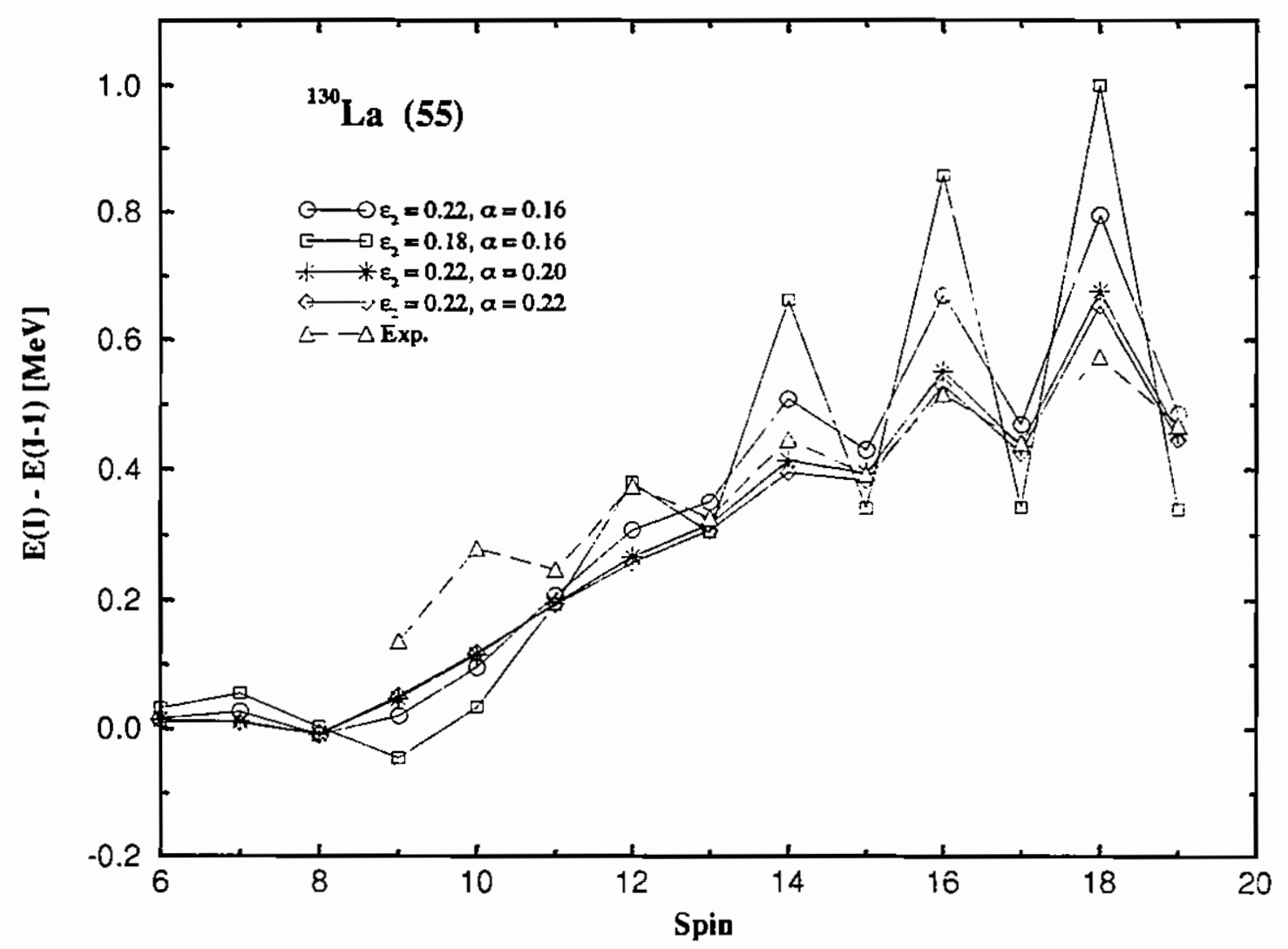

Figura 5.8: Comparaçāo dos cálculos teóricos com os dados experimentais da banda 1 de configuração $\nu h_{11 / 2} \otimes \pi h_{11 / 2}$ do núcleo ímpar-ímpar de ${ }^{130} L a$, para diferentes valores de $\epsilon_{2}$ e $\alpha$. 


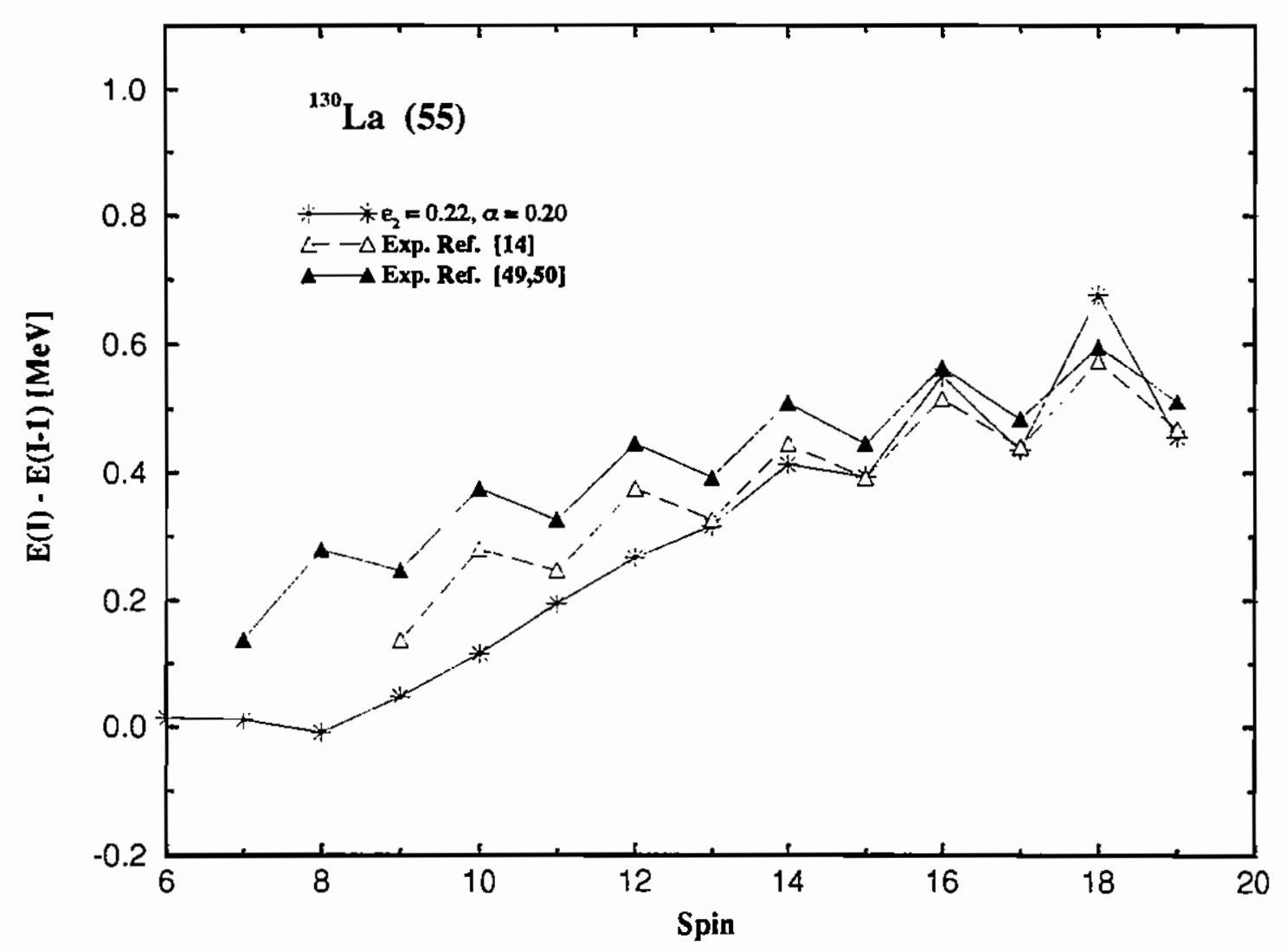

Figura 5.9: Comparação dos cálculos teóricos (asteriscos) para a banda 55 de ${ }^{130} \mathrm{La} \mathrm{com}$ os dados experimentais do laboratório Pelletron [14] (triângulo aberto) e do grupo de Liverpool $[49,50]$ (triângulo fechado).

às energias das transiçōes das bandas observadas no núcleo de ${ }^{130} \mathrm{La}$. A diferença principal entre os resultados de Liverpool e os outros dois trabalhos reside na atribuição do spin do cabeça de banda. Para determinar este spin o grupo do Pelletron e de Stony Brook usaram o acoplamento perpendicular, enquanto que o grupo de Liverpool usou a regra de Gallagher-Moszkowski. 
No diagrama de bandas correspondente (55) do núcleo de ${ }^{130} \mathrm{La}$ (figura 5.10 ) observase que a configuração $\left(h_{11 / 2}, h_{11 / 2}\right)$ é uma mistura de vários níveis. As contribuiçōes mais importantes sāo $\nu h_{11 / 2}(7 / 2) \otimes \pi h_{11 / 2}(3 / 2)$ e $\nu h_{11 / 2}(9 / 2) \otimes \pi h_{11 / 2}(5 / 2), K=-$ 5 e 7, respectivamente. Em spins mais altos as bandas com $K=3$ e 4 (configurações $\nu h_{11 / 2}(5 / 2) \otimes \pi h_{11 / 2}(1 / 2)$ e $\left.\nu h_{11 / 2}(5 / 2) \otimes \pi h_{11 / 2}(3 / 2)\right)$ se cruzam com a banda de $K=7$, e tornam-se então as responsáveis pelo pequeno "sttagering" que aparece na banda yrast. A banda com $K=1, \nu f_{7 / 2}(1 / 2) \otimes \pi h_{11 / 2}(1 / 2)$, com um pequeno "zigzag" de fase oposta, não deve ter muito efeito sobre a banda de yrast, por estar em energias mais altas.

De um modo similar, foram feitos os cálculos das bandas 54, 45 e 44 para o núcleo de ${ }^{130} \mathrm{La}$ onde os resultados para a banda 45 deste núcleo são mostrados na figura 5.11 . A tendência geral dos resultados experimentais é muito bem reproduzida, no entanto, em termos absolutos a concordância nāo é tão boa como no caso da banda 55. Novamente, podemos observar que a atribuição de spins feita pelo grupo do laboratório Pelletron [14] possui um melhor acordo com os dados experimentais do que a feita pelo grupo de Liverpool [49, 50]. Na banda 45 acima, os dadc:i experimentais não revelam nenhum "staggering" significativo. A análise do diagrama de bandas (45) correspondente, mostra que a estrutura desta banda consiste essencialmente de $\nu g_{7 / 2}(7 / 2) \otimes \pi h_{11 / 2}(3 / 2)$ e de $\nu g_{7 / 2}(7 / 2) \otimes \pi h_{11 / 2}(1 / 2) \operatorname{com} K=-5$ e -3 respectivamente.

Cálculos de bandas 44 e 54 não mostram nenhuma semelhança com os dados experimentais e deste modo foram descartados como candidatos para as configuraçōes das duas bandas observadas neste núcleo. Isto confirma as configuraçōes inicialmente atribuídas a estas bandas.

Os mesmos cálculos foram realizados para os núcleos de ${ }^{132} L a[4] \mathrm{e}^{132,134} \operatorname{Pr}[41,42,43]$ e resultados muito similares ao núcleo de ${ }^{130} \mathrm{La}$ foram obtidos para as bandas 55 e 45 , onde a concordância entre teoria e experiência para deformações prolatas é bastante satisfatória para spins acima de $12 \hbar$. As figuras 5.12, 5.13 e 5.14 mostram, respectivamente, os ajustes feitos para os dados experimentais. A falta de pontos experimentais de ${ }^{132} \mathrm{La}$ para spins mais altos faz com que seja menos significativa a comparaçāo com os resultados teóricos 


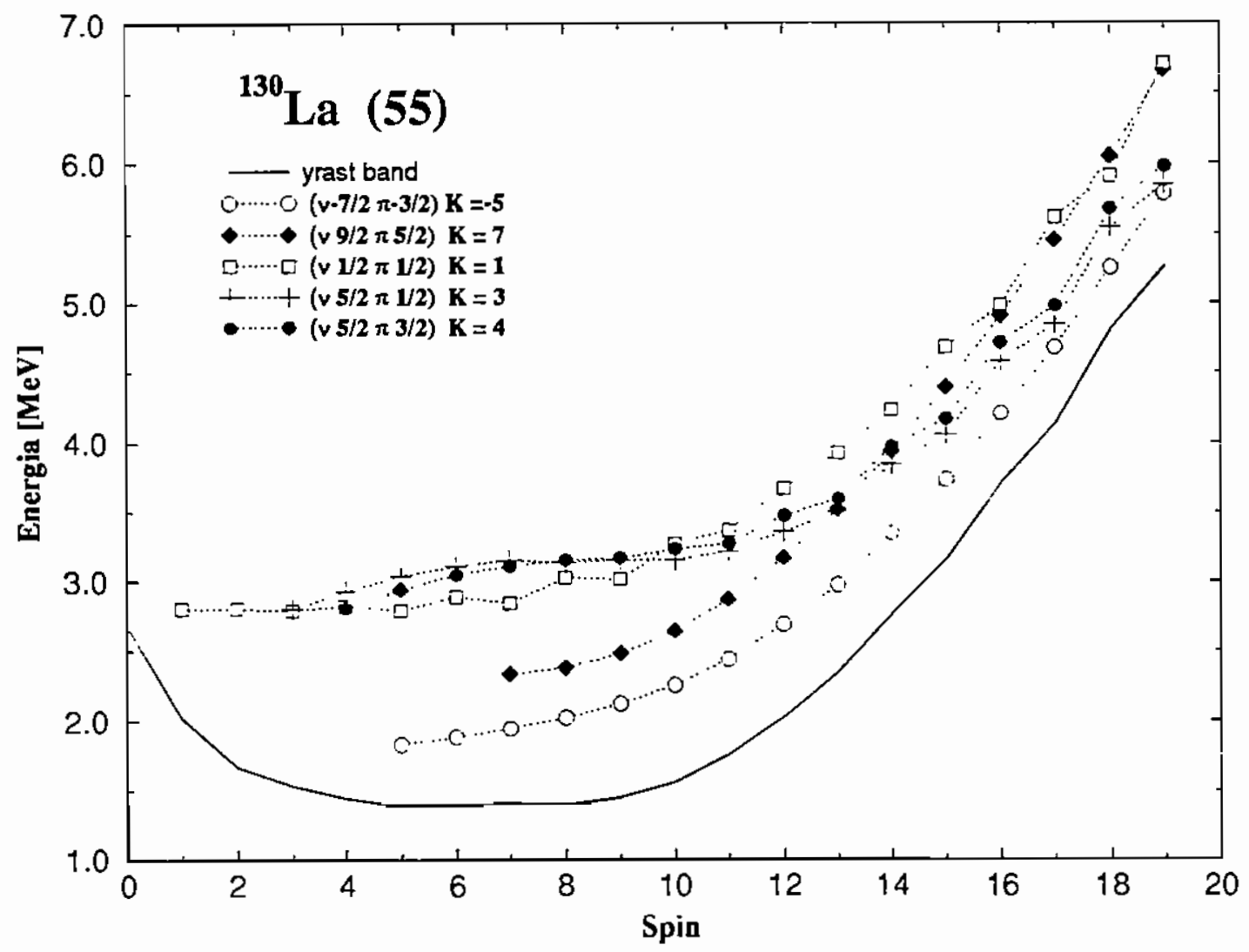

Figura 5.10: Diagrama de bandas para a configuraçāo $5 j$ de ${ }^{130} \mathrm{La}$. A banda yrast é mostrada com linha cheia para enfatizar o "staggering" em altos spins. Todas as projeçōes atribuídas e os correspondentes valores de $K$ pertencem à banda de configuraçāo $\nu h_{11 / 2} \otimes \pi h_{11 / 2}$ exceto para o valor de $K=1$ que é $\nu f_{7 / 2} \otimes \pi h_{11 / 2}$. 


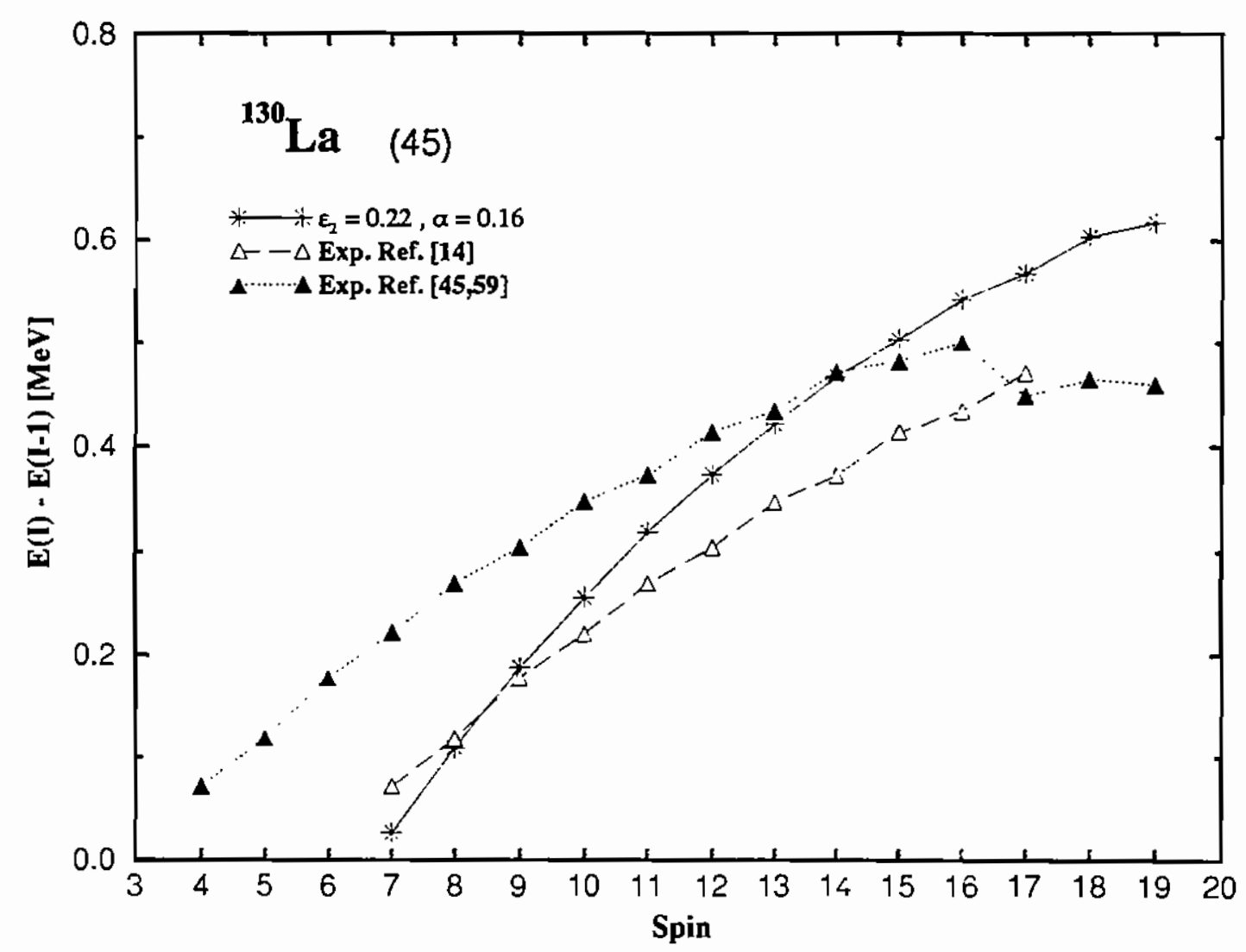

Figura 5.11: O mesmo que a figura 5.9, mas para a banda 45 do núcleo de ${ }^{130} \mathrm{La}$ de configuração $\nu g_{7 / 2} \otimes \pi h_{11 / 2}$. 
(figura 5.12) e, similarmente, nenhum cálculo teórico foi possível para a segunda banda deste núcleo devido ao pequeno número de pontos experimentais em altos spins. No caso do núcleo de ${ }^{134} \operatorname{Pr}$ [42] a banda 55 mostra também um acordo com os dados, porém, nenhum ajuste razoável foi possível para a segunda banda deste núcleo pelo fato de que esta banda somente foi observada acima do "backbend" com uma configuração de 4 quasepartículas.

b) $\mathbf{N}=77$

Os cálculos realizados para a banda 55 de ${ }^{136} \mathrm{Pr}$ [3] sāo mostrados na figura 5.15 , com os melhores resultados dos cálculos para valores de deformação $\beta$ positiva e negativa em comparação com os dados experimentais. Observa-se que as energias teóricas estão consistentemente abaixo dos dados experimentais. Para a segunda banda, o acordo entre teoria e experiência é ainda mais crítico. Situação similar ocorre para a banda 55 de seu isótono ${ }^{134} \mathrm{La}$ [3] (figura 5.16), mas isto pode ser devido à pequena quantidade de pontos experimentais.

Os cálculos realizados para núcleos vizinhos ímpares em $\mathrm{A}$, como ${ }^{137} \mathrm{Nd}[38],{ }^{135} \mathrm{Ce}$ [37], ${ }^{135,137} \operatorname{Pr}[7,8]$ e ${ }^{133} L a[9]$, também mostram um desacordo entre teoria e os dados experimentais. Isto pode ser devido ao fato de que nesta regiâo transicional a deformaçāo $\gamma$ deve ser necessária para a interpretaçāo dos dados experimentais, exigindo deste modo, um modelo que tenha o grau de liberdade $\gamma$ a ser ajustado. De fato, os núcleos de $\mathrm{Nd}$ e Ce que foram interpretados através do modelo de rotor triaxial mais partícula, mostram uma deformação $\gamma \approx-30^{\circ}([12]$ e $[10])$. 


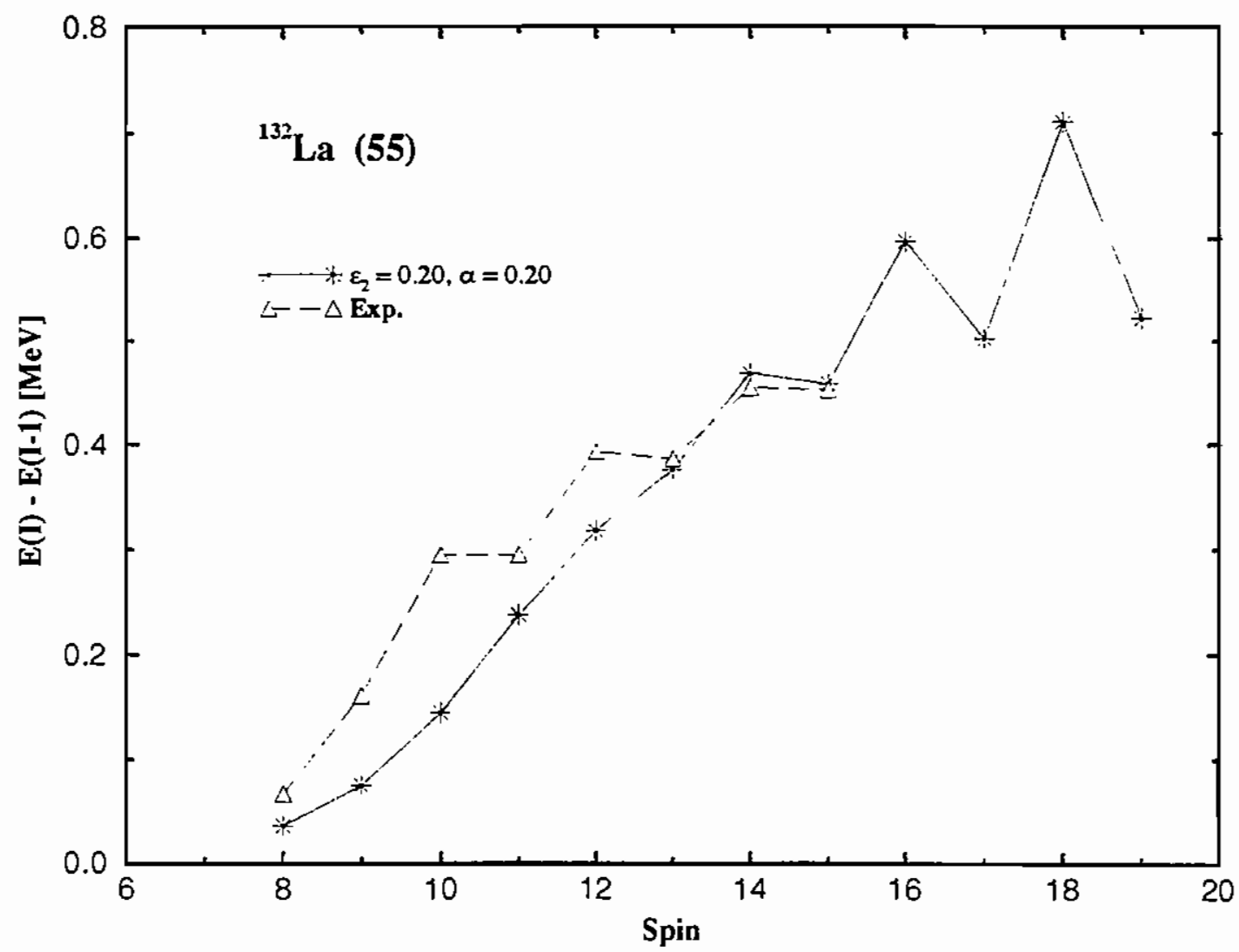

Figura 5.12: Comparação dos cálculos teóricos e experimentais para a banda 55 do núcleo impar-ímpar de ${ }^{132} \mathrm{La}$ [4]. 

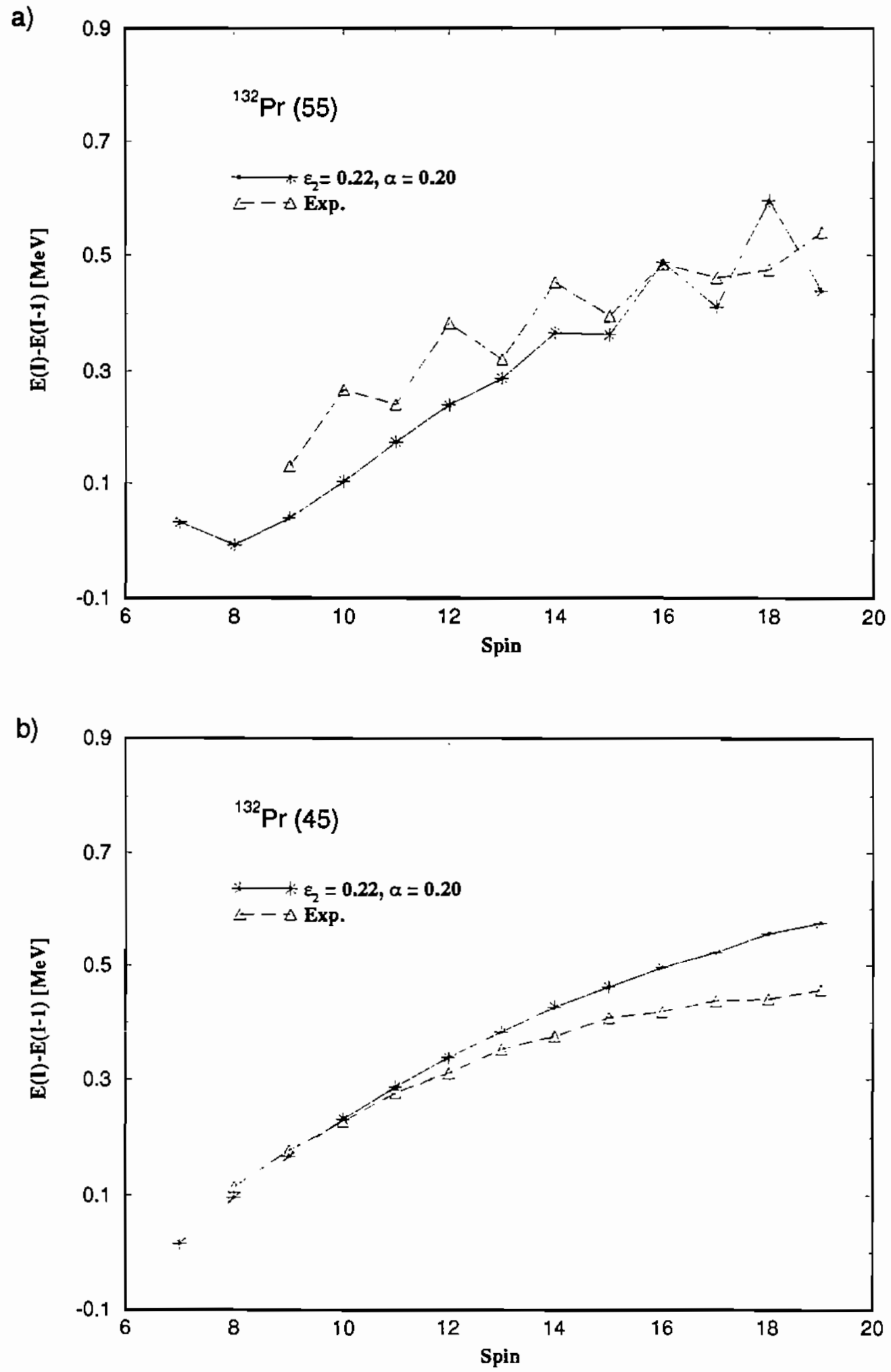

Figura 5.13: O mesmo que a figura 5.12, mas para a banda a) 55 e b) 45 do núcleo ímpar-ímpar de ${ }^{132} \operatorname{Pr}$ [41]. 


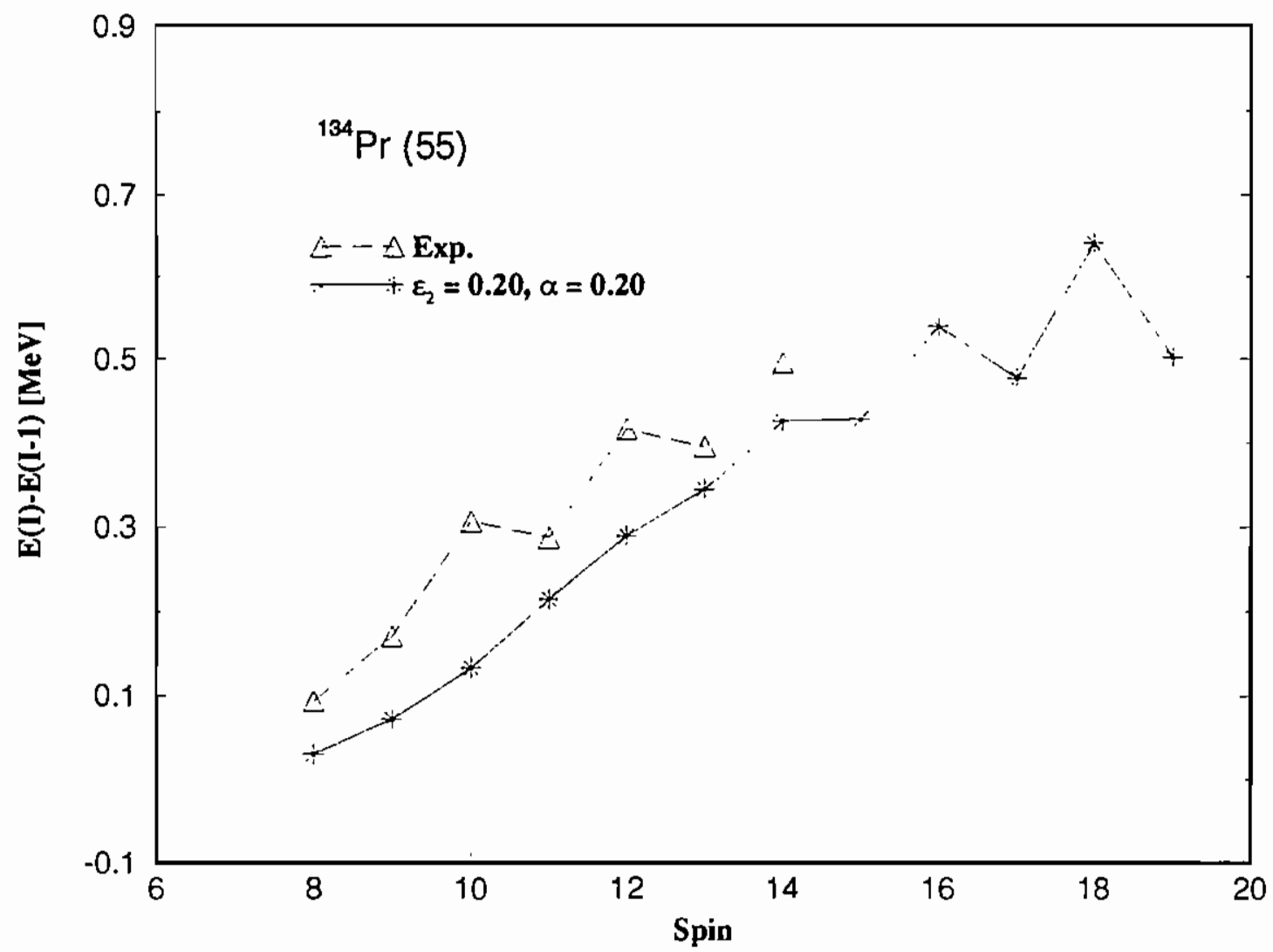

Figura 5.14: $O$ mesmo que a figura 5.12, mas para a banda principal de configuraçāo 55 do núcleo ímpar-ímpar de ${ }^{134} \operatorname{Pr}[42]$. 


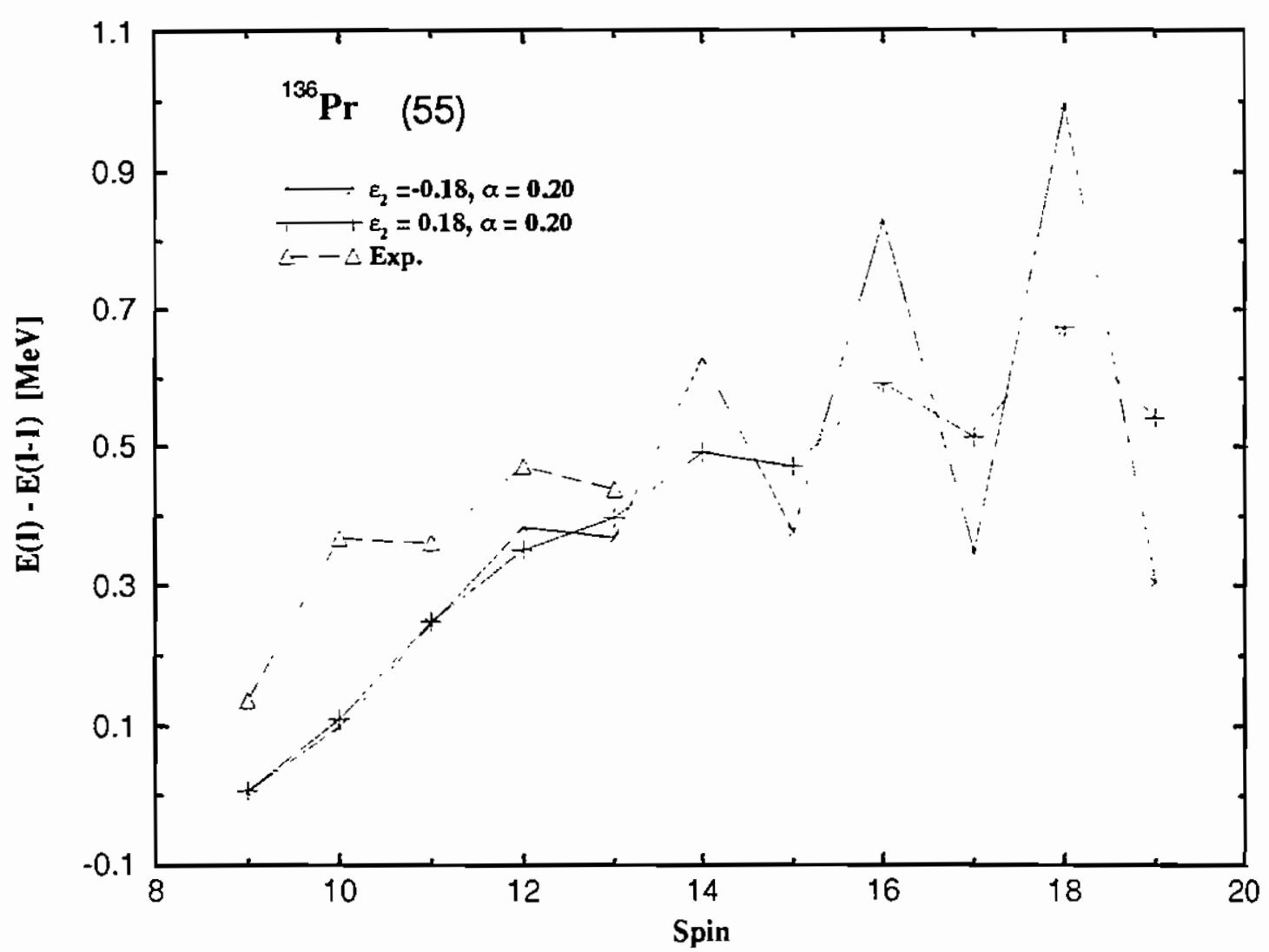

Figura 5.15: Cálculos teóricos para a banda 55 de ${ }^{136} \operatorname{Pr}$ para deformaçōes oblata $\epsilon_{2}=-0.18$ e prolata $\epsilon_{2}=0.18$ comparadas com os dados experimentais da referência [3]. 


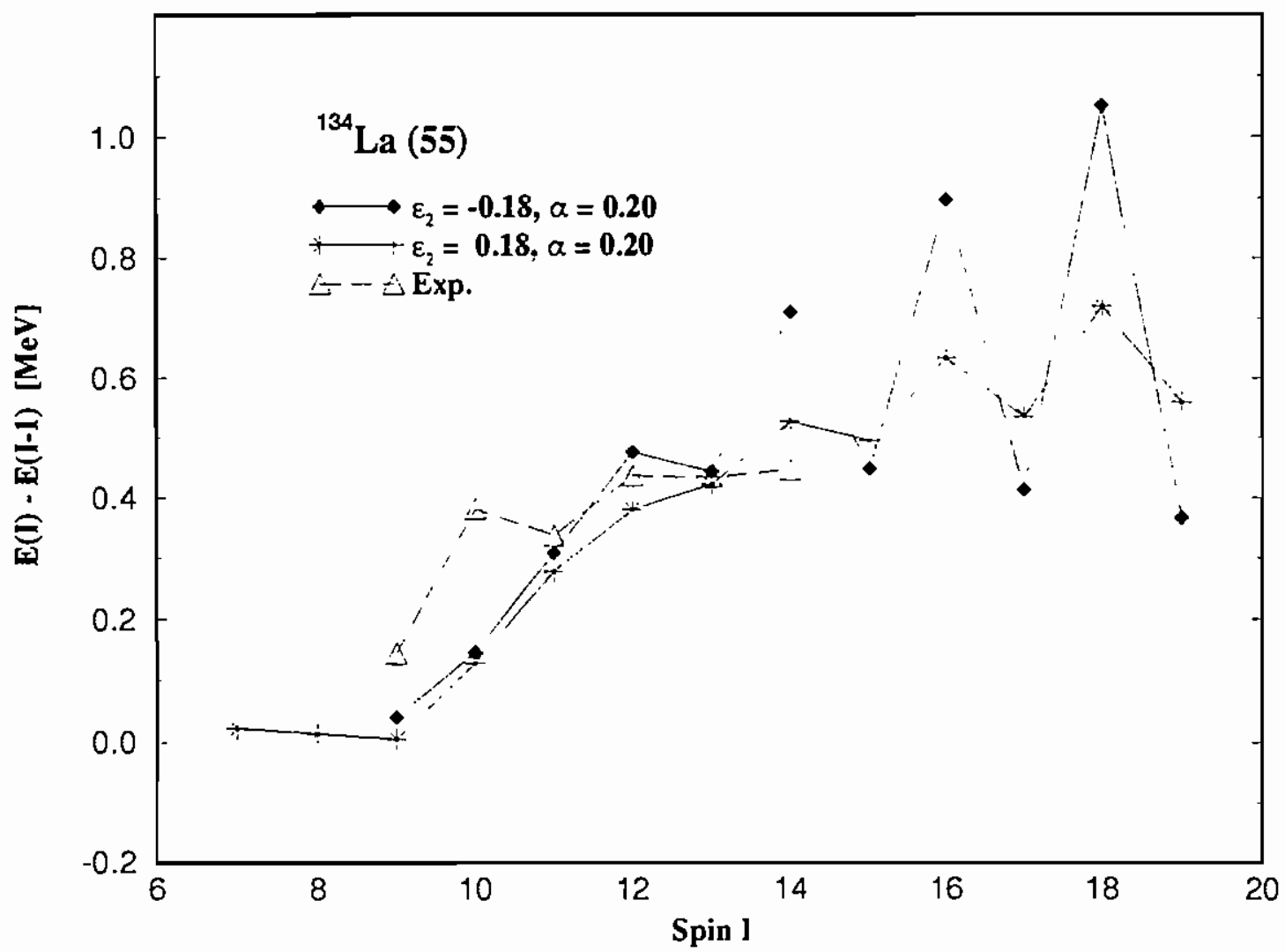

Figura 5.16: O mesmo que a figura 5.15 mas para o núcleo de ${ }^{134} \mathrm{La}$. Os dados experimenmiais sāo da referência [3]. 
c) $\mathbf{N}=\mathbf{7 9}$

Como foi discutido na seç̧ão 4.3 , a análise teórica do núcleo de ${ }^{138} P r$ mostrou que a banda de configuração $\nu h_{11 / 2} \otimes \pi h_{11 / 2}$ (55) possui um bom acordo, tanto para as deformaçōes positiva como negativa $\left(\epsilon_{2}=0.16\right)$, enquanto que para a banda 54 apenas os resultados com o valor de $\epsilon_{2}=-0.16$ são consistentes com os dados experimentais. Isto leva, talvez, a sugerir que o núcleo de ${ }^{138} \mathrm{Pr}$ é totalmente oblato, já que a coexistência de forma é pouco provável em energias de excitaçōes tão baixas [26].

Por outro lado, a coexistência de forma nāo pode ser descartada porque este modelo apenas conseguiu explicar uma das três bandas observadas experimentalmente. A banda 2 (2a e 2b), é importante para se entender esta coexistência ou não, mas infelizmente o código computacional do modelo de PSM utilizado aqui não trabalha com 4 quasi-partículas.

Finalizando, segue-se que, para os núcleos da região de $A \approx 130$ - 140, o modelo PSM sugere claramente uma transição de forma, de prolato $\left({ }^{130} \mathrm{La}, \mathrm{N}=73\right)$ para oblato $\left({ }^{138} \mathrm{Pr}\right.$, $N=79)$ passando através da forma provavelmente triaxial $\left({ }^{136} \mathrm{Pr}, \mathrm{N}=77\right)$, de acordo com as previsōes feitas nos núcleos ímpares de Ce e $\mathrm{Nd}$. O resumo destas conclusōes obtidas com o modelo de PSM é exposto na figura 5.17. 


\begin{tabular}{|c|c|c|c|c|c|c|c|}
\hline$z^{\prime}$ & 73 & 74 & 75 & 76 & $\pi 7$ & 78 & 79 \\
\hline 60 & $\begin{array}{l}{ }^{133} \mathrm{Nd} \\
\varepsilon_{2}=0.22 \\
\text { prolato }\end{array}$ & & $\begin{array}{l}{ }^{135} \mathbf{N d} \\
\varepsilon_{2}=0.20 \\
\text { probto }\end{array}$ & & $\begin{array}{c}{ }^{137} \mathbf{N d} \\
\varepsilon_{2}=0.17 \\
\star\end{array}$ & & $\begin{array}{r}{ }^{139} \mathrm{Nd} \\
\mathrm{E}_{2}-16 \\
\text { obbto }\end{array}$ \\
\hline 59 & $\begin{array}{l}{ }^{132} \mathrm{Pr} \\
\varepsilon_{2}=0.22 \\
\text { prolato }\end{array}$ & $\begin{array}{l}{ }^{133} \mathrm{Pr} \\
\varepsilon_{2}=0.21\end{array}$ & $\begin{array}{l}{ }^{134} \mathrm{Pr} \\
\varepsilon_{2}=0.20 \\
\text { probato }\end{array}$ & $\begin{array}{r}{ }^{135} \mathbf{P r} \\
\varepsilon_{2}=0.19 \\
*\end{array}$ & $\begin{array}{c}{ }^{136} \mathrm{Pr} \\
\varepsilon_{2}=0.18 \\
*\end{array}$ & $\begin{array}{c}{ }^{137} \mathrm{Pr} \\
\varepsilon_{2}=0.17 \\
*\end{array}$ & $\begin{array}{r}{ }^{138} \mathrm{Pr} \\
\varepsilon_{2}=-.16 \\
\text { obbtoto }\end{array}$ \\
\hline 58 & $\begin{array}{l}{ }^{131} \mathrm{Ce} \\
\varepsilon_{2}=0.22 \\
\text { probato }\end{array}$ & & $\begin{array}{r}{ }^{133} \mathrm{Ce} \\
\varepsilon_{2}=0.20 \\
\text { probto }\end{array}$ & & $\begin{array}{c}{ }^{135} \mathrm{Ce} \\
\varepsilon_{2}=0.18 \\
*\end{array}$ & & $\begin{array}{r}{ }^{137} \mathrm{Ce} \\
\varepsilon_{2}=-.16 \\
\text { obbato }\end{array}$ \\
\hline 57 & $\begin{array}{l}{ }^{130} \mathrm{La} \\
\varepsilon_{2}=0.22 \\
\text { protato }\end{array}$ & $\begin{array}{l}{ }^{131} \mathrm{La} \\
\varepsilon_{2}=0.21\end{array}$ & $\begin{array}{l}{ }^{132} \mathrm{La} \\
\varepsilon_{2}=0.20 \\
\text { probto }\end{array}$ & $\begin{array}{l}{ }^{133} \text { La } \\
\varepsilon_{2}=0.19\end{array}$ & $\begin{array}{l}{ }^{134} \mathbf{L a} \\
\varepsilon_{2}=0.18\end{array}$ & & \\
\hline
\end{tabular}

Figura 5.17: Sistemática de núcleos ímpares e ímpar-impar da região de massa $\mathrm{A}=\approx 130$ - 140, estudados com o modelo de CSM e PSM. 


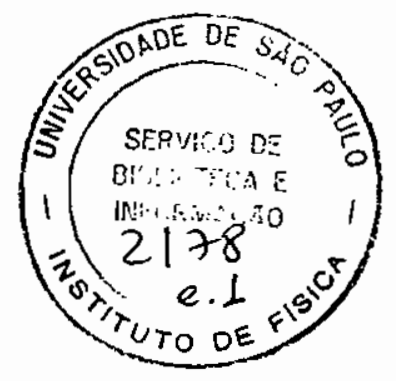




\section{Capítulo 6}

\section{Discussão e Conclusão}

A estrutura de alto-spin do núcleo duplamente ímpar de ${ }^{138} \mathrm{Pr}$ foi estudada pela primeira vez neste trabalho, onde várias bandas rotacionais foram observadas. Devese enfatizar que as únicas informaçōes conhecidas antes deste trabalho se resumiam ao conhecimento do estado fundamental $\left(I^{\pi}=1^{+}\right)$de meia vida de $1.4 m i n$. e de um estado isómerico $\left(I^{\pi}=7^{-}\right)$de meia vida de $2.1 h$ além de alguns estados de baixo spin provenientes do decaimento radioativo do ${ }^{138} \mathrm{Nd}$.

A banda yrast medida experimentalmente é. baseada na configuração $\pi h_{11 / 2} \otimes \nu h_{11 / 2}$ e apresenta um pequeno "staggering" com $\Delta e_{\text {exp }}^{\prime} \approx 50 \mathrm{keV}$ para a freqüêcia de $\approx 350$ $\mathrm{keV}$ que tende a zero para $\omega \approx 250 \mathrm{keV}$; e um alinhamento constante em torno de $6 \hbar$. Esta banda é bastante similar as bandas yrast observadas em vários vizinhos ímpar-ímpar. Em adição à banda yrast do ${ }^{138} \mathrm{Pr}$, foram populadas bandas secundárias com diferentes configuraçōes. Para a banda designada por 2 (banda 2a e 2b) atribuiu-se a configuraçāo $\pi[413] 5 / 2 \otimes \nu h_{11 / 2} \otimes\left(\pi h_{11 / 2}\right)^{2}$ que é a representação de uma banda de 4 quasi-partículas. A terceira banda possui transiçōes quadrupolares $(\Delta I=2)$ sendo sua configuração dada por $\pi[411] 1 / 2 \otimes \nu h_{11 / 2}$.

A análise dos dados de distribuição angular sugeriu que a maioria das transições pertencentes ao núcleo de ${ }^{138} \operatorname{Pr}$ possuem multipolaridade dipolar e poucos gamas sāo quadrupolares, sendo a banda yrast e a banda designada como 2 a constituídas basicamente de 
transições dipolares. As transições quadrupolares são fracas, exceto as que pertencem à banda 3. Os spins das bandas secundárias ( 2 e 3 ) foram tentativamente atribuídos de acordo com a configuração e a conexão destas bandas com a banda yrast.

O modelo de camadas de "Cranking" (CSM) e o modelo de camadas com projeção de momento angular (PSM) foram utilizados na interpretação dos dados experimentais deste trabalho. Os cálculos com CSM confirmaram as atribuiçōes das configurações das bandas medidas experimentalmente do ${ }^{138} \mathrm{Pr}$, principalmente no caso das banda $2 \mathrm{a}$ e $2 \mathrm{~b}$, com a configuração de 4 quasi-partículas. Além disto este modelo possibilitou sugerir as formas nucleares para as diversas bandas. Para a banda 2 ( $2 a$ e $2 b$ ), atribuiu-se a forma prolata e para a banda 3 a forma oblata, enquanto que para a banda $1, \pi h_{11 / 2} \otimes \nu h_{11 / 2}$, a forma final dependerá da escolha do caroço, isto é, de ${ }^{136} \mathrm{Ce}, \mathrm{Z}-1$ e $\mathrm{N}-1$, que é prolato ou de ${ }^{140} \mathrm{Nd}, \mathrm{Z}+1$ e $\mathrm{N}+1$, que é oblato.

Além disso, a análise dos dados com CSM mostra uma controvérsia sobre o grau de triaxialidade $\gamma$ dos núcleos duplamente ímpar da região de massa $A \approx 130-140$, dependendo do critério utilizado. A extração da deformação gama das curvas de e' $\mathbf{x}$ $\gamma$, para se obter o valor do "signature splitting" experimental, resulta em $\gamma \approx-20^{\circ}$, enquanto que a posição de equilibrio do routhiano total, indica valor de gama dentro do intervalo de $-5^{\circ}<\gamma<10^{\circ}$ e com valores de $\Delta e^{\prime} \approx 0 \mathrm{keV}$ para todos estes núcleos.

O método de projeção de momento angular, PSM, foi aplicado também no estudo do núcleo de ${ }^{138} \mathrm{Pr}$ e para os vizinhos ímpares e ímpar- ímpar desta região de massa com grande sucesso, permitindo obter as configurações detalhadas das bandas de interesse. Este modelo também sugeriu uma clara transição de forma em função do número de nêutrons, de prolato com $\mathrm{N}<75,{ }^{130} \mathrm{La}$, para oblato co $\mathrm{N}>79,{ }^{138} \mathrm{Pr}$.

A banda 3 de paridade negativa (54) de ${ }^{138} \mathrm{Pr}$ é um exemplo interessante da aplicabilidade de PSM. Cálculos com deformação prolata mostram espectros sem nenhuma similaridade com o esquema de níveis observado experimentalmente, enquanto que, com deformaçōes oblata o acordo além de ser muito bom evidencia uma degenerescência entre as duas "signatures" desta banda duplamente desacoplada. Isto nos coloca frente a novos desafios tanto do ponto de vista teórico quanto experimental; por um lado temos que bus- 
car justificativas teóricas para a eventual supressão de uma banda face à outra, enquanto que do ponto de vista experimental, teríamos que procurar indicaçōes de transiçōes dipolares fracas entre os membros das duas bandas, além de tentar medir as multipolaridades das transições abaixo da cabeça de banda (banda 3) e assim definir com maior confiança os respetivos spins.

Por outro lado, a teoria mostra que a banda de paridade positiva (55) de ${ }^{138} \mathrm{Pr}$ pode ser descrita assumindo-se tanto uma deformação prolata como oblata, devido à simetria em relação à deformação nula dos níveis de Nilsson para a camada $h_{1 \mathrm{I} / 2}$. No entanto, o código computacional usado aqui nāo permite efetuar cálculos para a banda de 4 quasipartículas (banda 2), que ajudaria a definir a forma nuclear. Assim, na base dos cálculos de PSM para as bandas 1 e 3 do ${ }^{138} \mathrm{Pr}$, e no fato que é pouco provável a coexistência de forma em energias tão baixas na região de $\mathrm{A} \approx 130$ - 140, é coerente atribuir ao núcleo de ${ }^{138} \operatorname{Pr}$ uma forma oblata.

Os cálculos sistemáticos para as bandas yrast desta região mostram ainda uma degenerescência nos estados em torno da cabeça de banda. E isto talvez seja devido à interação esquemática que não contém nenhuma força nêutron-próton (do tipo partículapartícula). Sendo assim, uma investigação experimental e teórica cuidadosa em torno dos níveis de cabeça de banda dos núcleos desta regiāo será de grande valia.

No geral, existe um razoável acordo entre os cálculos de PSM e a experiência para toda esta região. Este modelo consegue reproduzir "staggering" experimental para altos spins, mas falha ao reproduzir os de baixos spins. Outro aspecto a ser mencionado é que os espaçamentos teóricos dos níveis próximos à cabeça de banda são geralmente menores que os observados, e isto pode indicar novamente a falta da interação nêutron-próton ou simplesmente reflete o efeito da nāo conservação do número de partículas inerente à aproximação de BCS. Sendo assim, temos que projetar a base do modelo de camadas com bom número de partículas em adiçāo ao momento angular e espera-se em breve estender o modelo à este propósito.

Em resumo, há um consenso entre os dois modelos, que na regiāo de massa $A \approx 130$ - 140 existe uma transiçāo de forma de prolata para oblata. No entanto, eles diferem 
na interpretação do núcleo de ${ }^{138} \mathrm{Pr}$, onde o primeiro indica coexistência de forma e o segundo sugere deformação oblata para o núcleo inteiro. Sob este apecto, investigações mais profundas, tanto experimentais como teóricas são necessárias. 


\section{Referências}

[1] J. Ragnarsson, A. Sobiczewski, R.K. Sheline, S.E.Larsson and B. Nerlo-Pomorska Nucl. Phys. A233 (1974)329.

[2] Y. Chen, S. Frauendorf and G.A. Leander Phys. Rev. C28 (1983)2437.

[3] J.R.B. Oliveira, L.G.R. Emediato, E.W. Cybulska, R.V.Ribas, W.A. Seale, M.N. Rao, N.H. Medina, M.A. Rizzutto, S. Botelho and C.L. Lima Phys. Rev. C45 (1992)2740.

[4] J.R.B. Oliveira, L.G.R. Emediato, M.A. Rizzutto, R.V. Ribas, W.A. Seale, M.N. Rao, N.H. Medina, S. Botelho and E.W. Cybulska e refências internas Phys. Rev. C39 (1989)2250.

[5] G.A. Leander, S. Frauendorf and F.R. May Proceeding of the Conference on High Angular Properties of Nuclei - Oak Ridge, 1982, ed. N. R. Johnson (Harwood-Academic, N.Y., 1983), pg. 281.

[6] G. Anderson, S.E. Larsson, G. Leander, P. Moller, S.G. Nilsson, I. Ragnarsson, S. Aberg, R. Bengtsson, J. Dudek, B. Nerlo-Pomorska, K. Pomorski and Z. Szymanski Nucl. Phys. A268 (1976)205.

[7] T.M. Semkow, D.G. Sarantites, K. Honkanen, V. Abenante, L.A. Adler, et al. Phys. Rev. C34 (1986)523. 
[8] N.Xu, C.W. Beausang, R. Ma, E.S. Paul, W.F. Piel Jr., D.B. Fossan and L. Hildingson Phys. Rev. C39 (1989)1799.

[9] L. Hildingson, W. Klamra, Th. Lindblad, C.G. Linden, G. Sletten and G. Szekely Z. Phys. A338 (1991)125.

[10] M. Kortelahti, T. Komppa, M. Piiparinen, A. Pakkanen and R. Komu Phys. Scripta, vol. 27 (1983)166.

[11] M. Müller-Veggian, Y. Gono, R.M. Lieder, A. Neskakis and C. Mayer-Boricke Nucl. Phys. A304 (1978)1.

[12] M. Müller-Veggian, H. Beuscher, D.R. Haenni, R.M. Lieder, A. Neskakis and C. Mayer-Boricke Nucl. Phys. A344 (1980)89.

[13] M.A. Cardona, G. de Angelis, D. Bazzacco, M. de Poli and S. Lunardi Z. Phys. A340 (1991)345.

[14] M. A. Rizzutto Tese de Mestrado - Universidade de São Paulo - Instituto de Física (1989).

[15] K. Hara and Y. Sun - Nucl. Phys. A529 (1991) 445;

K. Hara and Y. Sun - Nucl. Phys. A531 (1991) 221;

K. Hara and Y. Sun - Nucl. Phys. A537 (1992) 77;

K. Hara and Y. Sun - Z. Phys. A339 (1991) 15.

[16] M.A. Rizzutto, E.W. Cybulska, V.R. Vanin, J.R.B. Oliveira, L.G.R. Emediato, R.V. Ribas, W.A. Seale, M.N. Rao, N.H. Medina, S. Botelho, J.C. Acquadro and C.L. Lima

Z. Phys. A344 (1992)221.

[17] M.A. Rizzutto, E.W. Cybulska, L.G.R. Emediato, N.H. Medina, R.V. Ribas, K.Hara and C.L. Lima à ser publicado em Nucl. Phys. 
[18] R. Bengtsson and J. D. Garret

The Cranking Model - Proceedings of the Nordic Winter School on Nuclear Physics Hemsedal, Norway (1983).

[19] C.J. Gallagher and S.A. Moszkowski

Phys. Rev. 111 (1958)1282.

[20] S.M. Harris

Phys. Rev. 138 (1965)B509.

[21] S. Frauendorf and F.R. May

Phys. Lett. 125B (1983)245.

[22] T. Bengtsson and I. Ragnarson

Nucl. Phys. A436 (1985)14.

[23] L. Grodzins

Phys. Lett. 2 (1962)88.

[24] J. Meyer-Ter-Vehn

Nucl. Phys. A249 (1975)111.

[25] K. Hara and S. Iwasaki

Nucl. Phys. A430 (1984)175.

[26] K. Hara

Nuclear Rotational States - Notas de Aula - IFUSP (1992).

[27] P. Ring and P. Schuck

The Nuclear Many-Body Problem - Springer-Verlag Inc., New York/Berlin (1980).

[28] A. Gavron

Phys. Rev. C21 (1980)230.

[29] A.B. Wittkower, R.P. Bastide, N.B. Brooks and P.H. Rose Phys. Letter 3 (1963)336. 
[30] O. Sala and G. Spalek

Nuclear Instr. Meth. 122 (1974)213.

[31] R. V. Ribas

Manual do Sistema SPM-CAMAC para Aquisição de Dados - IFUSP/P-812 (1989).

[32] CAMAC Tutorial Papers

Nucl. Sci. NS20 (1973) no. 2.

[33] Bill Milner

VAXPAK Programs ORNL - Private Communications- Oak Ridge National Laboratory - USA - 1986.

[34] C. M. Lederer and V. S. Shirley

Table of Isotopes (1978).

[35] V. R. Vanin and M. Aiche

Nuclear Instr. and Meth. in Phys. Research A284 (1989)452.

[36] M. Piiparinen, M. Kortelahti, A. Pakkanen, T. Komppa and R. Komu Nucl. Phys. A342 (1980)57.

[37] R. Ma, E.S. Paul, D.B. Fossan, Y. Liang, R. Wadsworth, I. Jenkins and P.J. Nolan Phys. Rev. C41 (1990)2624.

[38] J. Gizon, A. Gizon, M.R. Maier, R.M. Diamond, F.S. Stephens Nucl. Phys. A222 (1974)557.

[39] D. Bazzacco, S. Lunardi, G. Nardelli, M. De Poli and G. de Angelis Z. Phys. A335 (1990)363.

[40] R. Ma, E.S. Paul, S. Shi, C.W. Beausang, W.F. Piel Jr., N. Xu and D. B. Fossan Phys. Rev. C37 (1988)1926. 
[41] S. Shi, C.W. Beausang, D.B. Fossan, R. Ma, E.S. Paul and N. Xu Phys. Rev. C37 (1988)1478.

[42] C.W. Beausang, L. Hildingsson, E.S. Paul, W.F. Piel Jr., P.K. Weng, N. Xu and D. B. Fossan Phys. Rev. C36 (1987)1810.

[43] C.M. Petrache, G. de Angeli, D. Bucurescu, M. Ivascu, D. Bazzacco and S. Lunardi Z. Phys. A344 (1992)227.

[44] C.W. Beausang, P.K. Weng, R. Ma, E.S. Paul, W. F. Piel Jr., N. Xu and D.B. Fossan

Phys. Rev. C42 (1990)541.

[45] F. Dönau and S. Frauendorf

Proceeding of the Conference on High Angular Properties of Nuclei - Oak Ridge, 1982, ed. N. R. Johnson (Harwood-Academic, N.Y., 1983), pg. 143.

[46] J.R.B. Oliveira Tese de Doutorado - Universidade de São Paulo - Instituto de Física (1989).

[47] K. Hara

Comunicação Interna - IFUSP (1992).

[48] E.S. Paul, C.W. Beausang, D. B. Fossan, R. Ma, W.F. Piel Jr., and N. Xu Phys. Rev. Lett. 58 (1987)984.

[49] M.J.Godfrey, Y. He, I. Jenkin, A. Kirwan, P.J. Nolan, D.J. Thornley, S.M. Mullins and R. Wadsworth

J. Phys. G: Nucl. Part. Phys. 15 (1989)487.

[50] M.J.Godfrey, Y. He, I. Jenkin, A. Kirwan, P.J. Nolan, D.J. Thornley, S.M. Mullins and R. Wadsworth

J. Phys. G: Nucl. Part. Phys. 15 (1989)671. 
$\cdots+\cdots \quad . . \quad$. 